
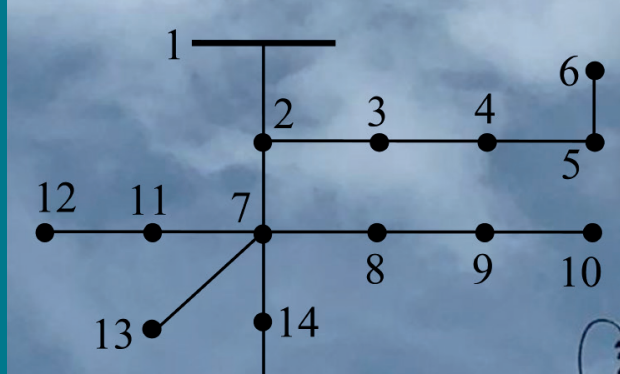
Oscar Gómez Carmona (Pereira, Risaralda, Colombia, 1979)

Ph. D, en Ingeniería, de la Universidad de Los Andes. Ingeniero Electricista, de la Universidad Tecnológica de Pereira. Profesor Titular de la Facultad de Tecnología de la Universidad Tecnológica de Pereira.

Autor de los libros: " On-line Monitoring of Electric Power System for Controlled Islanding" (2015) y " Proyección Operativa de Seguridad de Voltaje" (2009). Ha publicado artículos en revistas especializadas nacionales e internacionales .

Director del Grupo de Investigación Laboratorio de Investigación en Desarrollo Eléctrico y Electrónico (LIDER).

jr@utp.edu.co

Mauricio Granada Echeverri (Pereira, Risaralda, Colombia, 1975)

Ph. D. en Ingeniería Eléctrica de la Universidade Estadual Paulista UNESP -SP- Brasil, Ingeniero Electricista egresado de la Universidad Tecnológica de Pereira. Profesor Titular de la Facultad de Ingenierías de la Universidad Tecnológica de Pereira.

Autor de los libros: "Flujo de potencia óptimo en sistemas de transmisión de energía eléctrica" (2019), "Optimización combinatoria, de la teoría a la práctica" (2018), "Vehículos eléctricos, energía y movilidad" (2017), "Flujo de carga en sistemas de transmisión" (2016) y "Reducción de pérdidas técnicas en sistemas de distribución, aplicando medidas correctivas por etapas" (2006). Ha publicado artículos en revistas especializadas nacionales e internacionales.

Director del Grupo de Desarrollo en Investigación Operativa (DINOP).

magra@utp.edu.co

Carlo Julio Zapata Grisales (Cartago, Valle, Colombia, 1966)

Ph. D, en Ingeniería, de la Universidad de Los Andes. Ingeniero Electricista, de la Universidad Tecnológica de Pereira. Profesor Asociado de la Facultad de Tecnología de la Universidad Tecnológica de Pereira.

Ha publicado artículos en revistas especializadas nacionales e internacionales.

Integrante de los grupos de investigación Laboratorio de Investigación en Desarrollo Eléctrico y Electrónico (LIDER) y del Grupo de Desarrollo en Investigación Operativa (DINOP).

\section{Este libro pertenece a la Colección} Trabajos de Investigación. 


\title{
Pérdidas Técnicas en Redes Secundarias de Distribución de Energía Eléctrica
}

\author{
Oscar Gómez Carmona \\ Mauricio Granada Echeverri \\ Carlos Julio Zapata Grisales
}

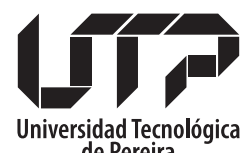
de Pereira

Colección Trabajos de Investigación

Facultad de Tecnología 2020 
Gómez Carmona, Oscar

Pérdidas técnicas en redes secundarias de distribución de

energía eléctrica / Oscar Gómez Carmona, Mauricio Granada

Echeverri y Carlos Julio Zapata Grisales. - Pereira :

Universidad Tecnológica de Pereira, 2020.

125 páginas. - (Colección Trabajos de investigación).

ISBN: 978-958-722-488-7

eISBN: 978-958-722-489-4

1. Sistemas de distribución - Energía eléctrica 2. Fallas -

Energía eléctrica 3. Flujo de carga 4. Pérdidas eléctricas 5.

Reguladores eléctricos 6. Medidores electromecánicos 7.

Medidores electrónicos

CDD. 621.319

Autores

(C) Oscar Gómez Carmona

(C) Mauricio Granada Echeverri

(C) Carlos Julio Zapata Grisales

Universidad Tecnológica de Pereira

Pereira, Colombia

\section{Proyecto de Investigación}

Herramienta computacional para el cálculo de pérdidas técnicas en sistemas secundarios de distribución, inscrito con el código 9-19-5

\section{Universidad Tecnológica de Pereira}

Vicerrectoría de Investigaciones, Innovación y Extensión

Editorial Universidad Tecnológica de Pereira

Pereira, Colombia

\section{Coordinador editorial:}

Luis Miguel Vargas Valencia

luismvargas@utp.edu.co

Teléfono 3137381

Edificio 9, Biblioteca Central "Jorge Roa Martínez"

Cra. 27 No. 10-02 Los Álamos, Pereira, Colombia

www.utp.edu.co

Montaje y producción:

David Restrepo Suarez.

Universidad Tecnológica de Pereira 


\section{Agradecimientos}

Los autores agradecen profundamente la colaboración de:

Darío Eliecer Rodas por sus aportes en el desarrollo conceptual de los modelos propuestos y las metodologías desarrolladas.

Víctor Mario Vélez y Daniel Felipe Castañeda por su colaboración en el desarrollo del software.

Paola Ríos por su colaboración en la edición del libro. 


\section{CONTENIDO}

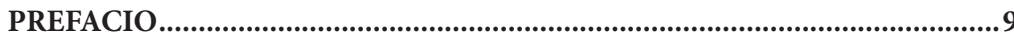

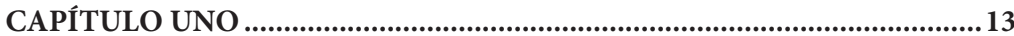

Pérdidas técnicas en sistemas de distribución ..................................................14

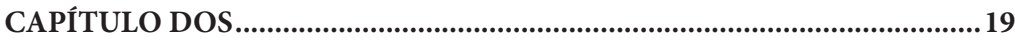

Modelamiento de componentes .........................................................................20

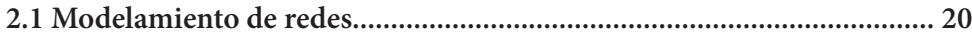

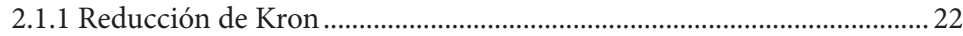

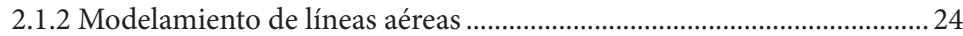

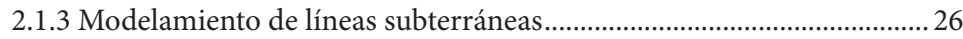

2.1.3.1 Neutro concéntrico con hilos ........................................................... 27

2.1.3.2 Cables con pantalla o cinta de cobre (semi-neutro)..................... 35

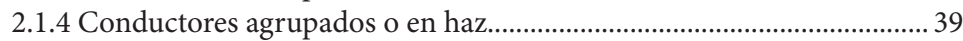

2.2 Modelamiento de cargas ..................................................................... 40

2.2.1 Modelamiento como impedancia constant ................................................. 45

2.2.2 Modelamiento como corriente constante.................................................. 46

2.2.3 Modelamiento como potencia constante: motores. ................................ 46

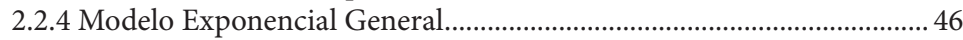

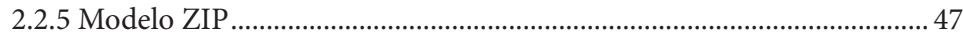

2.2.6 Impedancia constante versus potencia constante.................................... 48

2.3 Modelamiento de transformadores ................................................50

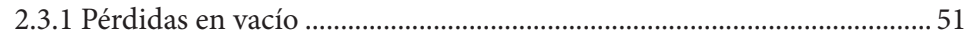

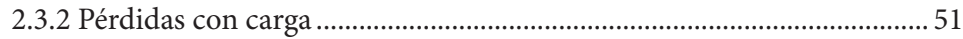

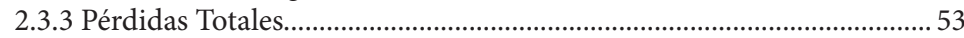

2.4 Modelamiento de medidores................................................................ 54

2.4.1 Consumo de los medidores electromecánicos y electrónicos según

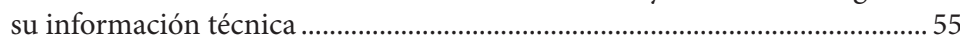

2.4.2 Consumo de los medidores electromecánicos y electrónicos según las normas NTC 2147, NTC 22882147 y NTC 4052 .......................................... 60

2.4.3 Análisis comparativo de los consumos de los medidores a partir

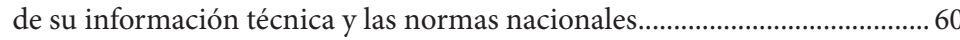

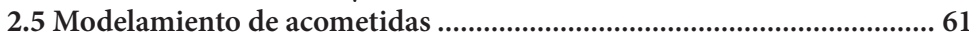

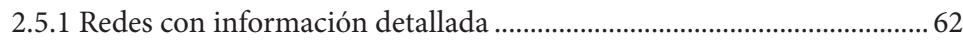

2.5.2 Redes donde solo se conoce la cantidad de usuarios por poste............. 62

2.5.3 Circuitos que no tienen red........................................................................ 63

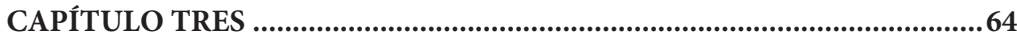

Metodología para el cálculo de pérdidas técnicas de redes radiales a partir de flujo de carga de barrido iterativo ..........................................................65

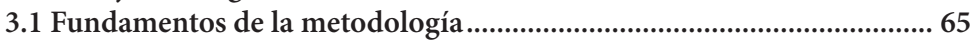

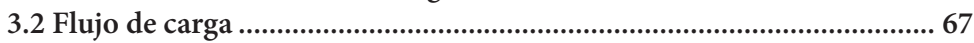

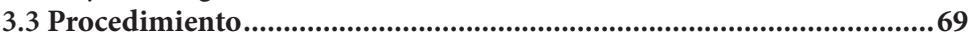

CAPÍTULO CUATRO ..............................................................................73

Metodología para el cálculo de pérdidas técnicas de redes en malla a partir de flujo de carga de barrido iterativo 


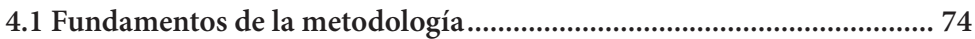

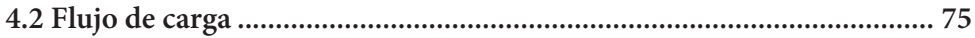

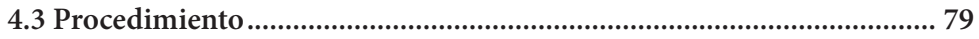

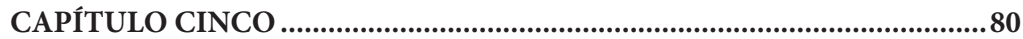

Herramientas computacionales desarrolladas ...................................................81

5.1 Diagrama de proceso ................................................................................. 82

5.2 Estructura de los datos de entrada ....................................................83

5.3 Procedimiento para revisar coherencia de los datos..............................87

5.4 Aplicativo para flujo de carga radial desbalanceado .............................89

5.5 Aplicativo para flujo de carga de red en malla .......................................90

5.6 Software y muestra de resultados ..............................................................92

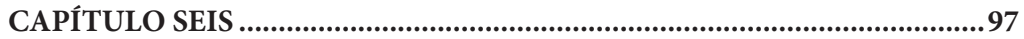

Ejemplos y casos de prueba ..................................................................................98

6.1 Circuito de prueba 1 .............................................................................. 99

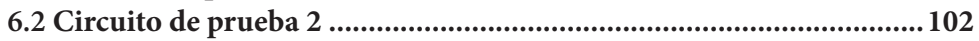

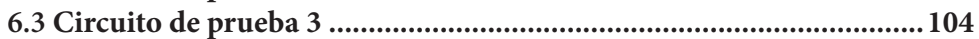

6.4 Circuito de prueba 4 ........................................................................... 107

6.5 Resultados a nivel del sistema de distribución secundaria .................110

Referencias bibliográficas ......................................................................115 


\section{TABLAS}

TABla NRo. 1. Primera configuración de las matrices de impedancia serie

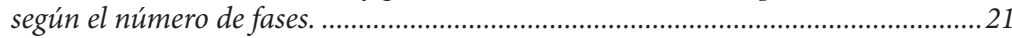

TABLA NRo. 2. Configuración de las matrices de admitancia shunt según el número de fases.

TABLA NRo. 3. Segunda configuración de las matrices de impedancia serie según el número de fases.

TABLA NRo. 4. Configuración de las matrices de admitancia shunt según el

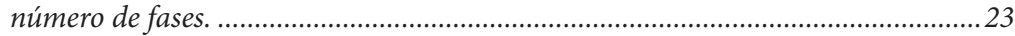

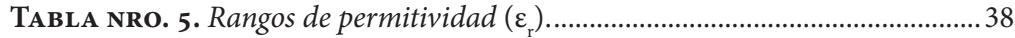

TABLA NRo. 6. Corriente de carga según tipo de conexión (PQ constante).......... 43

TABLA NRo. 7. Consumo de medidores electromecánicos para conexión directa de acuerdo con su tipo de conexión...........................................................55

TABLA NRo. 8. Consumo de medidores estáticos monofásicos bifilares para

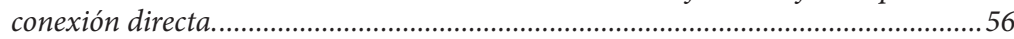

TABLA NRo. 9. Consumo de medidores estáticos bifásicos bifilares para

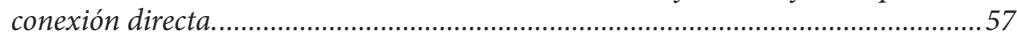

TABLA NRo. 10. Consumo de medidores estáticos trifásicos tetrafilares para

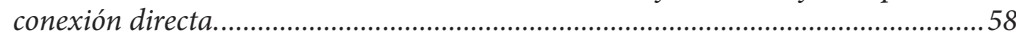

TABLA NRO. 11. Consumo de medidores estáticos trifásicos tetrafilares para

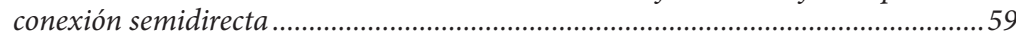

TABLA NRO. 12. Consumo de potencia en circuitos de tensión según las NTC

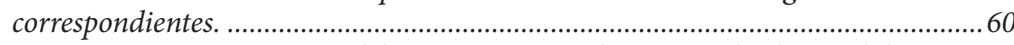

TABLA NRO. 13. Resumen del consumo promedio para todos los medidores versus consumo estipulado por la norma............................................................... 61

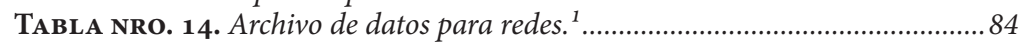

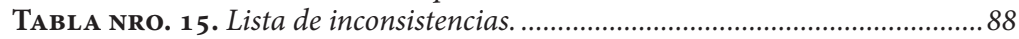

TABLA NRO. 16. Circuitos de prueba para cálculo de pérdidas técnicas. .............. 98

TABLA NRO. 17. Información de usuarios conectados al circuito 1.......................99

TABLA NRO. 18. Información de usuarios conectados al circuito 2......................102

TABLA NRO. 19. Información de usuarios conectados al circuito 3....................... 105

TABLA NRO. 20. Información de usuarios conectados al circuito 4.......................108

TABLA NRO. 21. Resultados de los circuitos para los días festivos y ordinarios... 111

TABLA NRO. 22. Pérdidas técnicas globales del sistema de distribución...............111

TABLA NRO. 23. Resultados de pérdidas técnicas por localización urbano/

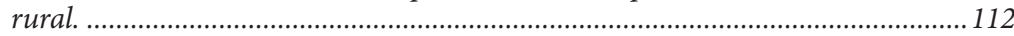

TABLA NRo. 24. Pérdidas técnicas para circuitos por tipo de transformador

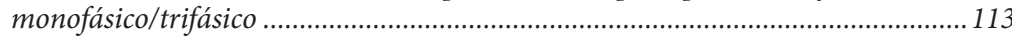

TABLA NRO. 25. Resultados de pérdidas técnicas por topología radial/mallado. 113

TABLA NRO. 26. Resultados de pérdidas técnicas por zona geográfica.................114 


\section{FIGURAS}

Figura NRo. 1. Modelo de la línea............................................................................20

FIGURA NRO. 2. Estructura del cable subterráneo.................................................2. 27

Figura NRo. 3. Sección transversal de un cable subterráneo.................................28

FigURA NRO. 4. Cable subterráneo con representación de neutro equivalente.....28

Figura NRO. 5. Cable subterráneo con representación de hilos del neutro concéntrico. ........................................................................................................ 33

Figura NRo. 6. Efecto capacitivo de la línea subterránea......................................35

FIGURA NRO. 7. Cables con pantalla o cinta de cobre ...........................................35

FIGURA NRO. 8. Disposición geométrica de los conductores.................................39

FIGURA NRO. 9. Conexiones de la carga en Y y $\Delta$..............................................4 42

FIGURA NRO. 10. Variación de la demanda ante cambios de voltaje para los

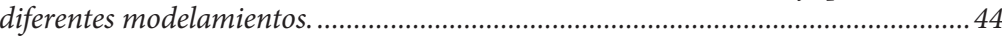

FIGURA NRO. 11. Circuito unifilar para modelar .................................................. 48

Figura NRO. 12. Pérdidas de potencia en vatios para el ejemplo.........................49

Figura NRo. 13. Porcentaje de pérdidas respecto de la fuente. .............................4 49

Figura NRo. 15. Diagrama de flujo de la metodología propuesta. ......................69

FigURA NRo. 16. Modelo trifásico de red secundaria en malla. ............................74

FIGURA NRO. 17. Diagrama del flujo de carga para redes en malla. ....................79

FIGURA NRO. 18. Herramientas computacionales para el cálculo de pérdidas

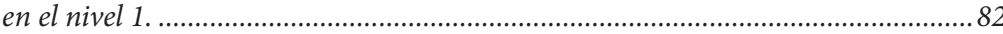

FIGURA NRO. 19. Botón y entorno gráfico para cargar la información en el software PESIDIBT UTP.

FIGURA NRO. 20. Entorno gráfico para cargar la información en el software PESIDIBT UTP.

FIGURA NRO. 21. Entorno gráfico en que se muestra la información cargada en el software PESIDIBT UTP.

Figura NRo. 22. Diagrama de flujo del proceso para revisar coherencia de los datos.

FIgURA NRO. 23. Visualización de DETERED UTP para ubicar inconsistencias en la información de circuitos en nivel de tensión 1........................89

FIgURA NRo. 24. Procedimiento del aplicativo para flujo de carga radial. ..........90

Figura NRo. 25. Procedimiento del aplicativo para flujo de red en malla.......... 91

FIGURA NRO. 26. Botón para realizar la ejecución del cálculo de pérdidas

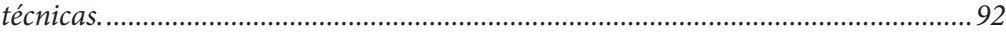

Figura NRO. 27. Ejecución del cálculo de pérdidas técnicas................................92

Figura NRO. 28. Botón y ventana de los resultados de pérdidas técnicas en nivel de tensión 1 .

Figura NRO. 29. Visualización de los resultados de pérdidas técnicas N1 por

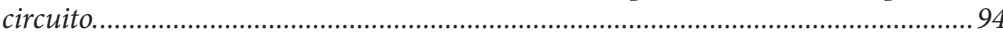

FIGURA NRO. 30. Visualización de los resultados totales de pérdidas técnicas N1.

Figura NRO. 31. Visualización de los resultados de pérdidas técnicas N1 por agrupación de localización, tipo de transformador, zona geográfica y municipio. 95 FIGURA NRO. 33. Topología del circuito 1 ...........................................................99

Figura NRo. 35. Energía anual y pérdidas técnicas del circuito 1. .....................101

Figura NRO. 36. Máxima cargabilidad y caída de tensión para el circuito 1....101

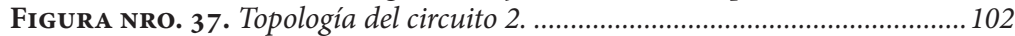

Figura NRo. 38. Parámetros descriptivos del circuito 2...................................... 103 
FIGURA NRO. 39. Energía anual y pérdidas técnicas del circuito 2..................... 103

FIgURA NRO. 40. Máxima cargabilidad y caída de tensión para el circuito 2....104

FIGURA NRO. 41. Topología del circuito 3. ........................................................ 105

FIGURA NRO. 42. Parámetros descriptivos del circuito 3....................................... 106

FIGURA NRo. 43. Energía anual y pérdidas técnicas del circuito 3. ....................106

FIGURA NRO. 44. Máxima cargabilidad y caída de tensión para el circuito 3....107

FIGURA NRO. 45. Topología del circuito 4. ........................................................ 108

FIGURA NRO. 46. Parámetros descriptivos del circuito 4..................................... 109

FIGURA NRO. 47. Energía anual y pérdidas técnicas del circuito 4....................109

FIGURA NRO. 48. Máxima cargabilidad y caída de tensión para el circuito 4...110

FIGURA NRO. 49. Pérdidas totales por locación.................................................112

FIGURA NRO. 50. Pérdidas totales por tipo de transformador. .............................113

FIGURA NRO. 51. Pérdidas totales por tipo de topología de red........................... 114 
PREFACIO

El sistema de suministro de energía eléctrica está conformado por varias etapas funcionales: generación, transmisión, subtransmisión y distribución de energía eléctrica. La generación transforma una forma de energía (hidráulica, térmica, solar, eólica, mareomotriz, etc.) en energía propiamente eléctrica. Esta es transportada a los centros de consumo a través de los sistemas de transmisión y subtransmisión; finalmente, los sistemas de distribución se encargan de llevar la energía eléctrica a todos los puntos de utilización con niveles de tensión adecuados.

Enlosprocesosdegeneración, transmisión,subtransmisión y distribución, hay energía consumida por los equipos que no es facturada al usuario final. La energía consumida por los dispositivos del sistema que no es aprovechable se denomina «pérdidas técnicas de energía». En general, se destacan dos tipos de pérdidas: 
a. Pérdidas óhmicas o pérdidas en el cobre: provocadas por la circulación de corriente eléctrica a través de los conductores. Su magnitud depende de las características de las redes y de la potencia demandada en un instante de tiempo. La magnitud de la disipación de energía depende en gran medida del patrón de carga del sistema, de los diferentes tipos de carga y del diseño de las líneas. Este tipo de pérdidas, aunque no se pueden eliminar, pueden ser reducidas.

b. Pérdidas en vacío: Estas se originan por la circulación de corrientes de magnetización en los núcleos de hierro de transformadores y otros equipos de la red. También se incluyen en esta categoría las pérdidas por corrientes parásitas en aisladores y por efecto corona; aunque estas se manifiestan fundamentalmente en redes de transmisión y en el sistema de distribución, son insignificantes. Las pérdidas de vacío aparecen cuando los circuitos están energizados, independientemente del nivel de carga.

Las pérdidas técnicas en el sistema de distribución han representado históricamente un alto costo a los usuarios y a las empresas prestadoras del servicio. En general, el comercializador debe pagar al generador y al transmisor el total de la energía que ingresa a su sistema, aunque esta no sea facturada a los usuarios. No obstante, los usuarios pagan un valor adicional al asociado a su consumo, ya que la tarifa incluye costos de pérdidas reconocidas en generación, transmisión y distribución.

Aunque los operadores de red tienen capacidad de gestión sobre las pérdidas técnicas, ya que son los encargados de la planeación y operación del sistema; no es económicamente viable diseñar un sistema con un porcentaje de pérdidas muy bajo, dado que eso implicaría niveles de inversión prohibitivos.

Uno de los objetivos de las empresas de distribución es reducir sus niveles de pérdidas técnicas con el objetivo de alcanzar la eficiencia en el uso de los recursos y racionalizar las 
necesidades de inversión en infraestructura eléctrica. El índice de dichas pérdidas es un indicador de la gestión técnica de una empresa distribuidora; por lo que conocer su valor es crucial para la definición de políticas de operación del sistema.

En Colombia, las medidas regulatorias tienen en cuenta las pérdidas que se presentan en el sistema de distribución, de tal forma que reconocen un determinado nivel de pérdidas y penalizan los excesos sobre él. La Comisión de Regulación de Energía y Gas (CREG), en [1], establece las condiciones para que los operadores de red inviertan nuevos recursos para modernizar la infraestructura utilizada en la actividad de distribución de energía eléctrica con el fin de mejorar la calidad, garantizar la prestación continua y eficiente del servicio, y disminuir las pérdidas de energía que hoy tiene el sistema.

El valor correspondiente a las pérdidas reconocidas a cada operador de red se incluye en el cargo por uso de los sistemas de distribución; por lo cual, el comercializador dentro de la tarifa recauda el valor de las pérdidas reconocidas y lo transfiere al operador de red.

En el «Capítulo 7» de [1], se definen los métodos para la determinación de los índices de pérdidas por nivel de tensión. En particular, las empresas de distribución que quieran calcular su índice de pérdidas deben modelar la red de la siguiente manera:

a. Se debe utilizar la información real de los transformadores y redes a través de los cuales se haya distribuido, como mínimo, el $80 \%$ de la energía vendida en el mercado de comercialización durante el año anterior a la presentación del estudio.

b. Para las áreas urbanas se deben modelar como mínimo el 90 $\%$ de los transformadores existentes. 
c. Se debe considerar un modelo de impedancia constante. En ningún punto del sistema se pueden presentar factores de potencia inferiores a 0.9 y los valores de voltaje no pueden ser inferiores a los establecidos en las normas vigentes.

d. La energía circulante en un año por las redes del nivel de tensión 1 no debe superar la energía de entrada a dicho nivel de tensión, declarada por el operador de red.

El operador de red debe presentar a la CREG la totalidad de la información base del cálculo.

Con el fin de poner a disposición de los operadores de red una herramienta computacional que les permita calcular su índice de pérdidas técnicas, se propuso el proyecto de desarrollo tecnológico, denominado «Herramienta computacional para el cálculo de pérdidas técnicas en sistemas secundarios de distribución», registrado en la Vicerrectoría de Investigación, Innovación y Extensión de la Universidad Tecnológica de Pereira con el código 9-19-5. Este trabajo fue desarrollado por los grupos de investigación Laboratorio de Investigación en Desarrollo Eléctrico y Electrónico - LIDER - y Desarrollo en Investigación de Operaciones -DINOP-.

Este libro tiene como finalidad divulgar los modelos matemáticos de los componentes del sistema de distribución secundario utilizados, las metodologías matemáticas desarrolladas que permiten calcular el índice de pérdidas técnicas en los sistemas de distribución secundarios radiales y en malla, y hacer una descripción de los aplicativos desarrollados para tal fin. 


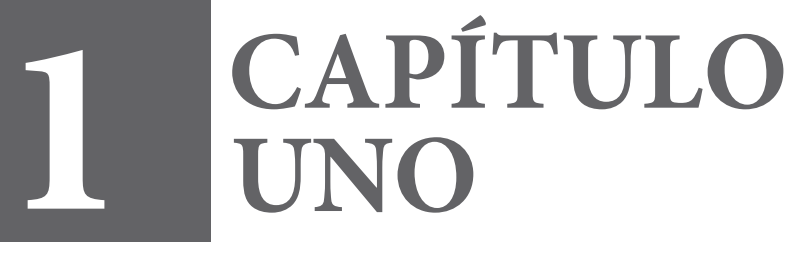




\section{Pérdidas técnicas en sistemas de distribución}

El sector eléctrico tiene tres áreas funcionales: generación, transmisión y distribución. La energía eléctrica es producida en las centrales generadoras. Estas convierten fuentes de energía primaria en energía eléctrica. El sistema de transmisión es la siguiente etapa de la cadena de suministro y se encarga de transportar la energía eléctrica desde las plantas de generación hasta los centros de distribución. Los sistemas de distribución tienen dos principales redes: de distribución primaria y secundaria. Las primeras alimentan grandes usuarios (conectados a media tensión) y transformadores de distribución. Los transformadores de distribución alimentan las redes de distribución secundaria, las cuales son el punto de conexión de los usuarios al sistema eléctrico.

Durante los procesos de generación, transmisión y distribución de energía eléctrica, hay energía que no es aprovechada por el usuario final y es disipada en dispositivos y lugares tales como líneas, núcleos de los transformadores y conexiones, entre otros. Esta energía que no es aprovechable se denomina "pérdidas de energía eléctrica».

Del total de estas pérdidas en el sector eléctrico, aproximadamente dos terceras partes corresponden a pérdidas técnicas, ocasionadas en conductores y transformadores del sistema eléctrico. Una tercera parte corresponde a pérdidas no técnicas ocasionadas por fraude, errores de medición y errores en la facturación. Cerca de un $70 \%$ de las pérdidas técnicas ocurre en las redes de distribución, específicamente, en conductores de los circuitos primarios y secundarios, y en los transformadores de distribución. Las pérdidas técnicas dependen de las características y operación de la red, y se pueden clasificar en pérdidas técnicas fijas y pérdidas técnicas variables [2]. 
Las pérdidas técnicas fijas no están influenciadas por la cantidad de corriente de carga que fluye. Estas se presentan en forma de calor y ruido, y se producen cada vez que se energizan los equipos. Entre el $25 \%$ y $30 \%$ de las pérdidas técnicas son pérdidas fijas y normalmente se presentan en las siguientes formas:

a. Pérdidas por efecto corona.

b. Pérdidas por corrientes de fuga.

c. Pérdidas dieléctricas.

d. Pérdidas de circuito abierto.

e. Pérdidas causadas por la carga de elementos de medición.

f. Pérdidas causadas por la carga de elementos de control.

Por otra parte, entre el $70 \%$ y $75 \%$ de pérdidas técnicas son pérdidas técnicas variables, las cuales son proporcionales al cuadrado de la corriente de carga y surgen debido a la impedancia de la línea, las resistencias de contacto y las pérdidas por calentamiento. Si se aumenta el área de la sección transversal de los conductores, este tipo de pérdidas disminuye; por lo tanto, las empresas de distribución deben encontrar un punto de equilibrio entre el costo de las pérdidas técnicas y los costos de inversión en infraestructura eléctrica.

En general, entre los factores que contribuyen a las altas pérdidas técnicas en los sistemas de distribución se encuentran:

- Selección inadecuada de transformadores: los transformadores de distribución tienen devanados con conductores de cobre que inducen un campo magnético en un núcleo de acero. Por lo tanto, los transformadores tienen pérdidas técnicas fijas y pérdidas técnicas variables. 
- Bajos factores de potencia y altos flujos de reactivos: si el factor de potencia es bajo, la corriente hacia la carga aumenta y las pérdidas técnicas aumentan con el cuadrado de esta corriente. La presencia de sistemas de aire acondicionado, ventiladores, sistemas de refrigeración y cargas industriales en áreas urbanas que comparten la red secundaria, sumado en ocasiones a estaciones de bombeo que usan motores eléctricos en áreas rurales, hace que el sistema eléctrico tenga bajo factor de potencia lo cual conlleva a mayores pérdidas. En caso de existir bajos factores de potencia, se deben establecer políticas de mejoramiento. Aumentar el factor de potencia produce una reducción del porcentaje de pérdidas.

- Transformadores de distribución que no están localizados en el centro de carga: muchas veces los transformadores de distribución no son ubicados centralmente respecto a los usuarios; por lo tanto, los usuarios ubicados más lejos experimentan bajos voltajes, así en bornes del transformador haya buenos niveles de tensión. Estas caídas de tensión incrementan las pérdidas técnicas en el circuito de distribución secundaria. Las pérdidas en los transformadores de distribución se pueden reducir optimizando su capacidad, determinando el rango de mejor rendimiento, o intercambiando transformadores que se encuentren sobrecargados o cerca de su límite por aquellos que están sobredimensionados.

- Circuitos degranlongitud:las redes de distribución primaria y secundaria, normalmente en áreas rurales, son radiales y de gran longitud dado que alimentan cargas altamente dispersas. Como resultado, se obtiene altas resistencias por unidad de longitud en las líneas y consecuentemente altas pérdidas del tipo $\mathrm{I}^{2} \mathrm{R}$. En el caso de las redes de distribución secundaria deben establecerse límites a la longitud relativa de las redes, ya que los circuitos de gran longitud relativa producen mayores pérdidas porcentuales. 
- Inadecuada distribución de los flujos de potencia y altos niveles de desbalance de las corrientes en las fases del sistema: balancear las cargas del sistema de distribución disminuye las caídas de tensión por fase del sistema, mejora los perfiles de tensión de los usuarios y disminuye las pérdidas técnicas.

- Redes de distribución con calibres inadecuados: usualmente los conductores se seleccionan de acuerdo con la carga que se va a alimentar y la regulación máxima permitida en los puntos de carga; sin embargo, en la selección de los conductores de circuitos de gran longitud y con cargas altamente dispersas debe considerarse el efecto de las pérdidas técnicas.

- Gestión de carga: se requiere de un manejo adecuado de la demanda máxima diaria que existe en el sistema, ya que la demanda máxima puede ser diferente en los usuarios que se alimentan del sistema. Este criterio puede asociarse a un proceso de optimización que realiza una reconfiguración de un sistema favoreciendo la simultaneidad, en un mismo circuito, de cargas que se complementan respecto a la demanda máxima; es decir, cargas que presenten demandas máximas en tiempos diferentes. También, deben generarse políticas de incentivo para las cargas industriales que comparten redes con cargas residenciales con el propósito de desplazar sus curvas de carga de tal forma que favorezcan la reducción de la demanda máxima y permitan aumentar el factor de carga.

Las pérdidas técnicas, aunque no se pueden eliminar, pueden ser reducidas. Si estas pérdidas son reducidas, las redes disminuirán sus niveles de cargabilidad y consecuentemente los usuarios mejorarán sus perfiles de tensión. 
Con el fin de iniciar un programa de reducción de pérdidas técnicas, se debe conocer el estado actual de las pérdidas del sistema. Para esto, se deben utilizar modelos adecuados de cada uno de los elementos que hacen parte del sistema, incluyendo el transformador, la red de distribución, los medidores, las acometidas y las cargas. Adicionalmente, se debe utilizar una metodología de flujo de carga que permita determinar las condiciones de operación en régimen permanente (estado estacionario) del sistema y considerar las características particulares que tienen las redes de distribución secundarias.

Los sistemas de distribución secundaria son típicamente radiales; por lo tanto, el flujo de potencia nace en un nodo. Este nodo se reconoce como el transformador de distribución, lugar donde se reduce la tensión del nivel de media tensión al de baja tensión. En la red secundaria, se pueden encontrar muchos tipos de conexiones, ya que la mayoría de las cargas son residenciales; por lo tanto, el sistema de distribución secundaria es por naturaleza desbalanceado, aunque el desequilibro trata de amortiguarse repartiendo equitativamente las cargas en las tres fases (sistemas alimentados por transformadores trifásicos) o entre las dos fases (sistemas alimentados por transformadores monofásicos). Otro aspecto particular de los sistemas de distribución secundaria es la presencia de cargas de distinta naturaleza; por ejemplo, cargas residenciales, comerciales y de pequeña industria.

Estas particularidades de los sistemas de distribución secundaria hacen necesario desarrollar flujos de potencia especializados para estos sistemas, los cuales caractericen fielmente el sistema y utilicen la información que normalmente tienen las empresas acerca de sus sistemas. 


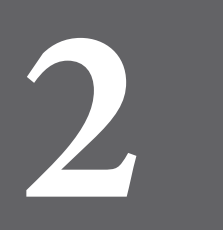

CAPÍTULO DOS 


\section{Modelamiento de componentes}

\subsection{Modelamiento de redes}

El modelo de las líneas de distribución tanto aéreas como subterráneas es importante dado que hace parte de los elementos que interconectan los nodos y permiten la transmisión de energía por la red. En el análisis, es importante incluir el espaciamiento entre conductores y el cálculo de las matrices de impedancia y admitancia de fase sin asumir transposición en las líneas.

El modelo de la línea se construye matricialmente porque en sistemas de distribución no se realiza transposición, lo que da una importancia significativa a los enlaces de flujo para cada fase. El sistema generalmente está desbalanceado, por lo cual, el modelo de la línea que se realiza es trifásico.

El modelo para la línea se compone de una matriz de impedancia-serie de tamaño $(n \times n)$, y de las matrices de capacitancia en derivación de tamaño $(n \times n)$, donde $n$ representa el número de conductores que posee la línea, tal como se muestra en la Figura NRO. 1.

Figura Nro. 1. Modelo de la línea.

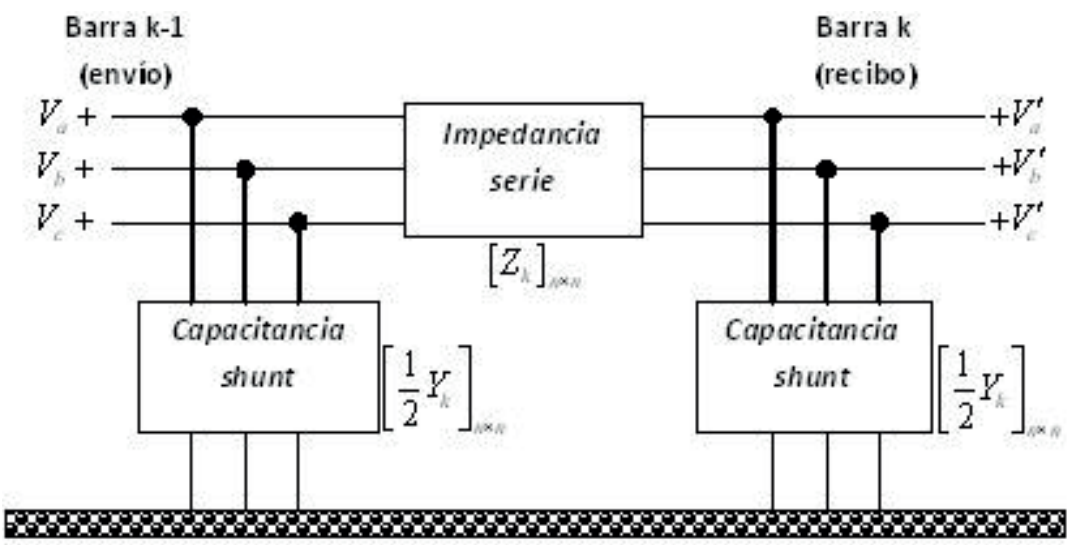


Las redes del sistema de distribución secundaria se modelan en forma trifásica; es decir, en todos los casos, las impedancias de los diferentes tramos son matrices de $3 \times 3$. Se toma en cuenta el efecto de Carson y el retorno por tierra, y a los conductores de neutro se les hace reducción por el método de Kron.

Para el análisis trifásico, las líneas se modelan como una impedancia. La matriz de impedancia-serie tendrá un tamaño fijo de $3 \times 3$ en concordancia con las características de los sistemas de distribución, independientemente del número de conductores por fase y neutros que se tengan. Siempre que se exceda este tamaño, se hace reducción de Kron, elemento a elemento, ya que los neutros están aterrizados sólidamente [3].

Una vez realizada la reducción de Kron, la estructura de las matrices cambia de acuerdo a la configuración que tenga la línea, es decir, conforme al número de fases de la línea y al número de conductores por fase (si tiene neutro o no)xe "reducción de Kron"xe "Kron" \t "(ver) reducción de Kron"; tal y como se muestra en las tablas nro. 1 y 2 [4].

TABLA NRO. 1. Primera configuración de las matrices de impedancia serie según el número de fases.

\begin{tabular}{|c|c|c|c|}
\hline Tres fases & Dos fases y un neutro & Una fase y un neutro \\
\hline$Z]=\left[\begin{array}{ccc}Z_{\mathrm{ii}} & Z_{\mathrm{ij}} & Z_{\mathrm{ik}} \\
Z_{\mathrm{ji}} & Z_{\mathrm{jj}} & Z_{\mathrm{jk}} \\
Z_{\mathrm{ki}} & Z_{\mathrm{kj}} & Z_{\mathrm{kk}}\end{array}\right]$ & {$[\mathrm{Z}]=\left[\begin{array}{ccc}Z_{\mathrm{ii}} & Z_{\mathrm{ij}} & - \\
Z_{\mathrm{ji}} & Z_{\mathrm{jj}} & - \\
- & - & -\end{array}\right]$} & {$[Z]=\left[\begin{array}{ccc}Z_{i i} & - \\
- & - & - \\
- & - & -\end{array}\right]$} \\
\hline
\end{tabular}

TABlA NRO. 2. Configuración de las matrices de admitancia shunt según el número de fases.

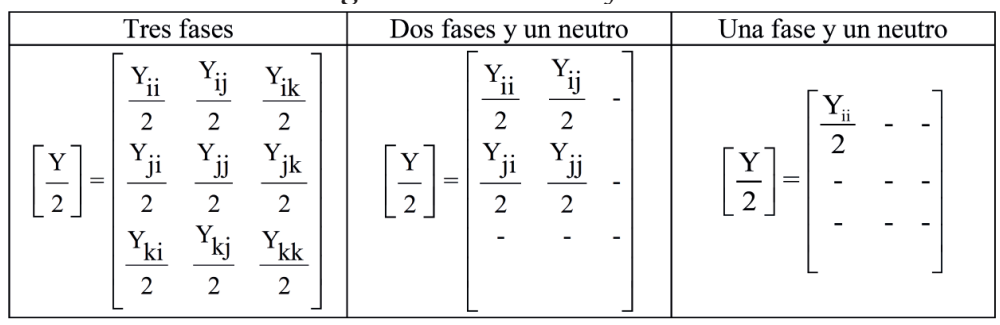


El efecto capacitivo de las redes es despreciable en este nivel de tensión. Cuando los tramos son trifásicos la matriz de impedancia es totalmente llena, de lo contrario, la matriz tendrá ceros en las fases que no se encuentren presentes en el tramo. Por ejemplo, si un tramo de línea solo tiene las fases $a$ y $c$, la forma de la matriz es:

$$
[\mathrm{Z}]=\left[\begin{array}{ccc}
\mathrm{Z}_{\mathrm{aa}} & 0 & \mathrm{Z}_{\mathrm{ac}} \\
0 & 0 & 0 \\
\mathrm{Z}_{\mathrm{ac}} & 0 & \mathrm{Z}_{\mathrm{cc}}
\end{array}\right]
$$

Para los circuitos sin información de red, estos se consideran con carga concentrada en el transformador; por lo tanto, no se les calcula las pérdidas en la red, y solo se calculan pérdidas en los medidores, acometidas y transformador de acuerdo con los modelos presentados en las siguientes secciones.

\subsubsection{Reducción de Kron}

Conforme a la configuración que tenga la línea, es decir, el número de fases de la línea, el número de conductores por fase, si tiene neutro y los cables de guarda - los cuales se consideran puestos a tierra ambos-, se realiza la reducción de Kron de acuerdo con:

$$
\left[\begin{array}{c}
V_{a b c} \\
0
\end{array}\right]=\left[\begin{array}{cc}
Z_{p p} & Z_{p n} \\
Z_{n p} & Z_{n n}
\end{array}\right]\left[\begin{array}{c}
I_{a b c} \\
I_{n}
\end{array}\right]+\left[\begin{array}{c}
V_{a b c}^{\prime} \\
0
\end{array}\right]
$$

Allí, las siguientes submatrices de impedancia corresponden a:

$$
\begin{array}{ll}
Z_{p p}: & \text { conductores de fases. } \\
Z_{p n}: & \text { mutua de conductores de fases (neutros). } \\
Z_{n p}: & \text { mutua de conductores neutros (fases). } \\
Z_{n n}: & \text { conductores neutros. }
\end{array}
$$


Las tensiones de fases en las cargas se designan como tensiones primadas; las corrientes de las fases son $I_{a b c}$ y las de los neutros y/o guardas, $I_{n}$.

Se despeja $\left[I_{n}\right]$ de la segunda línea, se reemplaza en la primera y se obtiene:

$$
\begin{gathered}
V_{a b c}=\left(Z_{p p}-Z_{p n}\left[Z_{n n}\right]^{-1} Z_{n p}\right) I_{a b c}+V_{a b c}^{\prime} \\
Z_{\text {reducida }}=\left(Z_{p p}-Z_{p n}\left[Z_{n n}\right]^{-1} Z_{n p}\right)
\end{gathered}
$$

Después de realizada la reducción de Kron, 1 XE "reducción de Kron" XE "Kron" It "(ver) reducción de Kron" a estructura de las matrices adopta la forma ilustrada en las tablas nro. 3 y 4 , según sea el número de fases que existan en la red.

TABLA NRO. 3. Segunda configuración de las matrices de impedancia serie según el número de fases.

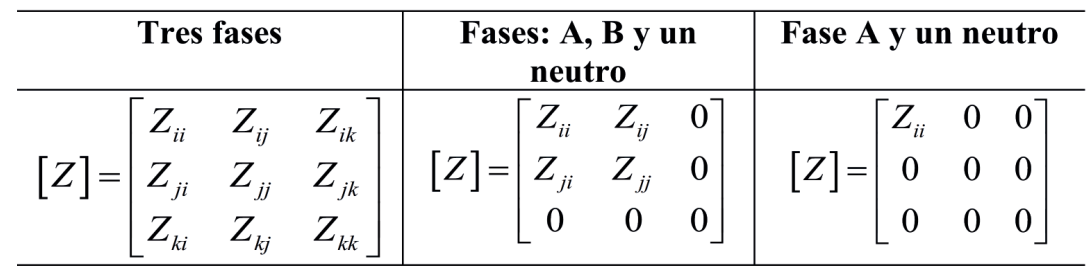

TABLa NRo. 4. Configuración de las matrices de admitancia shunt según el número de fases.

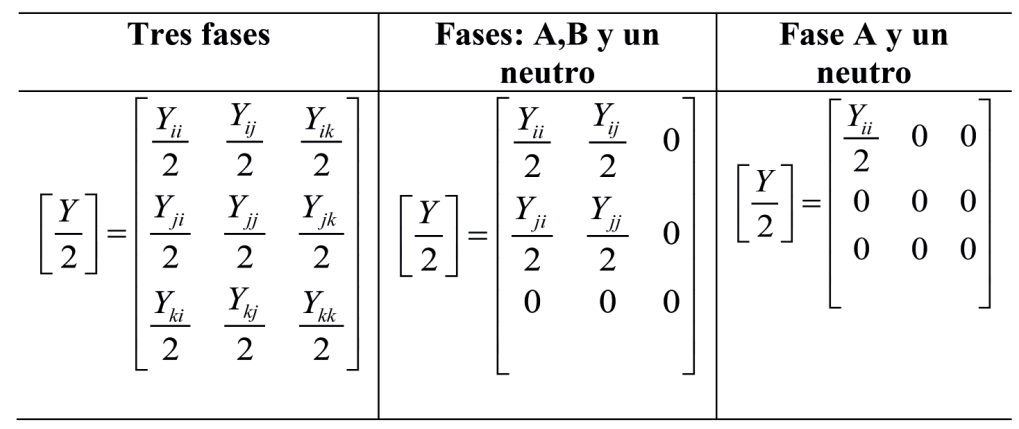


En los casos de una o dos fases, diferentes a las mostradas en las tablas nro. 3 y 4 , se deberán ubicar los valores de impedancia $\mathrm{o}$ admitancia en las posiciones adecuadas.

\subsubsection{Modelamiento de líneas aéreas}

Los conductores de las líneas aéreas son expuestos al aire y no poseen recubrimiento o aislante. En el modelamiento de líneas de transmisión, es de especial importancia el efecto inductivo y el efecto capacitivo; mientras que el modelo de la línea aérea del sistema de distribución se basa en la impedancia-serie, dado que el efecto capacitivo (admitancia shunt) es despreciado por varias causas, entre ellas:

- Las líneas en los sistemas de distribución son cortas.

- El nivel de tensión es relativamente bajo.

- Espaciamiento apreciable entre conductores, y entre conductores y tierra.

El efecto de los enlaces de flujo XE "efecto de los enlaces del flujo" (que es el causante de las impedancias mutuas) y el efecto del terreno XE "efecto del terreno" es tenido en cuenta en los elementos que conforman la matriz $[\mathrm{Z}]_{\text {serie }}$. Esta es simétrica con respecto a la diagonal y a la cuadrada.

La matriz impedancia-serie XE "líneas aéreas: matriz impedancia serie" del modelo, sin tener el efecto del terreno, es:

$$
\left[Z_{p r i m}\right]=\left[\begin{array}{ccccc}
z_{i i} & \cdots & z_{i k} & \cdots & z_{i j} \\
\vdots & \ddots & \vdots & \vdots & \vdots \\
z_{k i} & \cdots & z_{k k} & \cdots & z_{k j} \\
\vdots & \vdots & \vdots & \ddots & \vdots \\
z_{j i} & \cdots & z_{j k} & \cdots & z_{j j}
\end{array}\right]
$$


Con

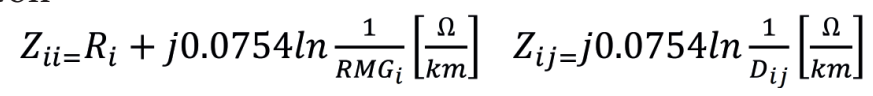

Donde

$Z_{i i}$ : elemento de la diagonal (Impedancia propia), en $[\Omega / \mathrm{km}]$.

$Z_{i j}$ : elemento fuera de la diagonal (Impedancia mutua), en $[\Omega / \mathrm{km}]$.

$R_{i}$ : $\quad$ resistencia propia del conductor, valor especificados por el fabricante para $\mathrm{T}=75^{\circ} \mathrm{C}$, en $[\Omega / \mathrm{km}]$.

$R M G_{i}$ : radio medio geométrico del conductor $i$, en $[\mathrm{m}]$.

$D_{i j}$ : distancia del conductor $i$ al conductor $j$, en $[\mathrm{m}]$.

Al ser necesario asumir la tierra como conductor de retorno, es preciso observar el efecto del terreno, ya que se producen enlaces de flujo por causa de esta corriente. Esto modifica los valores de impedancia propia e impedancia mutua de los conductores, es decir, los elementos de la diagonal y los de fuera de ella. Luego de hacer uso de la corrección de Carson, y sumar nuevos términos relacionados con la impedancia del terreno y las impedancias mutuas del terreno con las fases, este efecto produce:

$$
\begin{aligned}
& Z_{i i}=R_{i}+0.00098696 \cdot f+j 0.075 \cdot \ln \frac{D_{e}}{R M G_{i}}\left[\frac{\Omega}{k m}\right] \\
& Z_{i j}=0.00098696 \cdot f+j 0.0754 \cdot \ln \frac{D_{e}}{D_{i j}}\left[\frac{\Omega}{k m}\right]
\end{aligned}
$$

Donde

$$
D_{e}=658.5 \sqrt{\frac{\rho}{f}}[\mathrm{~m}] .
$$

$\rho: \quad$ resistividad del terreno, en $[\Omega-\mathrm{m}]$.

$f: \quad$ frecuencia de la red, en $[\mathrm{Hz}]$. 
Las expresiones (2.7) y (2.8) permiten calcular los elementos de la diagonal y por fuera de ella, llevando implícito el efecto del terreno. De tal modo, los valores asociados al terreno dependen solamente de la resistividad del terreno y la frecuencia de la red; generalmente, se trabaja con una resistividad de 100 $\Omega$-m, y a la frecuencia de $60 \mathrm{~Hz}$.

Para el caso general de una línea aérea trifásica con fases $a$, $b$, c y un neutro $n$, la matriz impedancia primitiva serie XE "líneas aéreas: matriz impedancia primitiva serie" XE "impedancia primitiva serie" toma la forma:

$$
[\text { Zprim }]_{\text {serie }}=\left[\begin{array}{llll}
Z_{a a} & Z_{a b} & Z_{a c} & Z_{a n} \\
Z_{b a} & Z_{b b} & Z_{b c} & Z_{b n} \\
Z_{c a} & Z_{c b} & Z_{c c} & Z_{c n} \\
Z_{n a} & Z_{n b} & Z_{n c} & Z_{n n}
\end{array}\right]
$$

Allí, los elementos que la conforman se calculan con las expresiones (2.7) y (2.8).

Dado que esta matriz es de tamaño $4 \times 4$, es preciso efectuar la reducción de Kron usando 2.1.1.

\subsubsection{Modelamiento de líneas subterráneas}

Los sistemas de distribución subterráneos poseen características especiales las cuales hacen que su modelamiento sea diferente. Por ejemplo, el efecto capacitivo en las líneas subterráneas tiene alguna incidencia debido a la cercanía entre conductores y a la proximidad entre estos y tierra. Adicionalmente, los conductores empleados para este tipo de línea tienen recubrimientos especiales y un neutro concéntrico, el cual está constituido por una capa o serie de hilos trenzados - como se muestra en la Figura NRO. 2-, o en forma de cinta - 
como se muestra en la Figura NRO. 7-. Este neutro va integrado a cada cable o cada fase donde el conductor para esta fase puede ser de cobre o de aluminio.

\subsubsection{Neutro concéntrico con hilos}

FIGURA NRO. 2. Estructura del cable subterráneo.

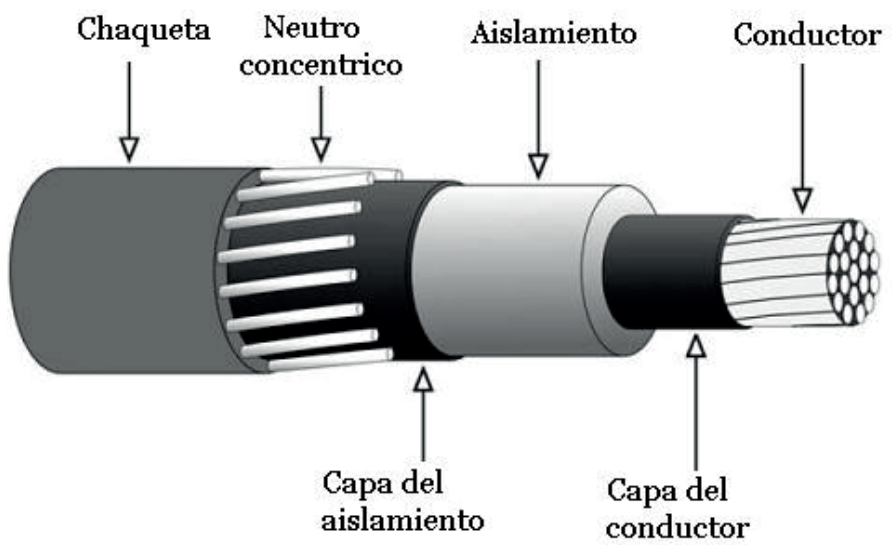

Así, en un sistema trifásico, se tienen tres neutros concéntricos XE "neutros concéntricos" e implica una matriz de impedancia primitiva de dimensiones $6 \times 6$. En el modelamiento de esta matriz, se utilizan las expresiones (2.7) y (2.8) para elementos de la diagonal (impedancia propia XE "impedancia propia" ) y de fuera de la diagonal (impedancia mutua XE "impedancia mutua"), obtenidos anteriormente para la línea aérea, con los cambios pertinentes para este caso. Estos se verán a continuación.

En las figuras nro. 2 y 3 se observa que el neutro concéntrico está conformado por varios hilos. A fin de simplificar el tamaño y el cálculo de la matriz impedancia, estos hilos o delgados conductores son representados por un neutro equivalente XE "sección transversal del conductor subterráneo" (ver FigurA NRO. 4). 
Figura NRo. 3. Sección transversal de un cable subterráneo.

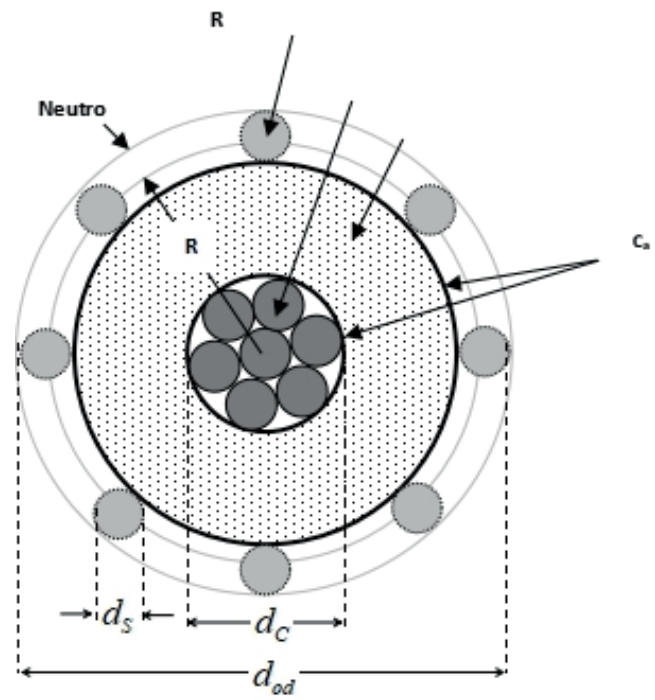

FIGURA NRO. 4. Cable subterráneo con representación de neutro equivalente.

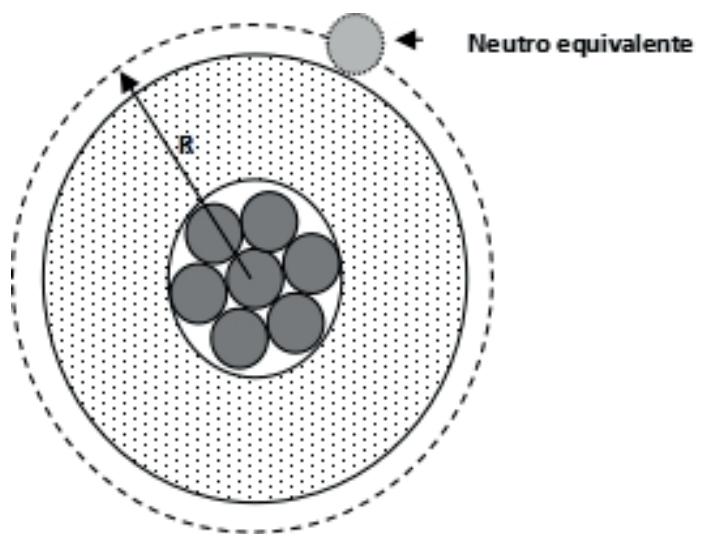

Buscando establecer el orden que tendrá cada conductor y su neutro concéntrico en la matriz de impedancia primitiva, se enumeran primero las fases y seguidamente los neutros concéntricos. Así, para un sistema general trifásico se obtiene: fases $1,2,3$ y neutros concéntricos 4, 5, 6 donde la fase 1 y el neutro concéntrico 4 estarán asociados al primer cable. La misma estrategia se aplica a los demás conductores. 
Se deben definir los parámetros de distancia que permiten el cálculo de los elementos de la matriz.

$G M R_{c n}$ es el radio medio geométrico XE "radio medio geométrico" del neutro concéntrico equivalente.

$$
G M R_{c n}=\sqrt[k]{G M R_{s} k R^{k-1}}[\mathrm{~m}]
$$

Donde

$R$ es la radio del círculo que atraviesa el centro de los hilos concéntricos, en metros (ver Figura NRO. 3 y Figura NRO. 4).

$k$ : $\quad$ número de hilos que conforman el neutro. $G M R_{s}$ : radio medio geométrico de un hilo del neutro, en $[\mathrm{m}]$.

De la disposición geométrica es fácil determinar las distancias entre los conductores de fase, como se hace en líneas aéreas.

La distancia entre un neutro concéntrico y su conductor de fase asociado es $\mathrm{R}$, en [m].

La distancia entre neutros concéntricos de dos cables se toma en forma aproximada a la distancia que tienen los conductores de fase respectivos.

La distancia entre un neutro concéntrico y el conductor de fase adyacente corresponde a $D_{i m}$. 


$$
D_{i m}=\sqrt[k]{D_{n m}^{k}-R^{k}} \approx D_{n m}[\mathrm{~m}]
$$

Donde

$$
\begin{aligned}
D_{n m}: & \text { distancia de centro a centro entre los } \\
& \text { conductores de fase correspondiente al } \\
& \text { neutro concéntrico y la fase adyacente. }
\end{aligned}
$$

La expresión (2.11) establece que la distancia entre un neutro y una fase vecina es, en forma aproximada, la distancia que hay entre las fases involucradas.

$R_{c n}: \quad$ resistencia equivalente del neutro concéntrico, en $[\Omega / \mathrm{km}]$.

$$
R_{c n}=\frac{R s}{k}\left[\frac{\Omega}{k m}\right]
$$

Donde,

$$
R_{s} \text { : es la resistencia de un hilo del neutro, en }[\Omega / \mathrm{km}] \text {. }
$$

Determinados los parámetros se calculan los elementos de la matriz de impedancia con (2.7) y (2.8). Para el caso trifásico, esta matriz de $6 \times 6$ tiene la siguiente estructura:

$$
\left[Z_{\text {prim }}\right]_{\text {serie }}=\left(\begin{array}{cccccc}
z_{11} & z_{12} & z_{13} & z_{14} & z_{15} & z_{16} \\
z_{21} & z_{22} & z_{23} & z_{24} & z_{25} & z_{26} \\
z_{31} & z_{32} & z_{33} & z_{34} & z_{35} & z_{36} \\
z_{41} & z_{42} & z_{43} & z_{44} & z_{45} & z_{46} \\
z_{51} & z_{52} & z_{53} & z_{54} & z_{55} & z_{56} \\
z_{61} & z_{62} & z_{63} & z_{64} & z_{65} & z_{66}
\end{array}\right)
$$


Si se reescribe la matriz, se tiene:

$$
\left[\begin{array}{cc}
Z_{p p} & Z_{p n} \\
Z_{n p} & Z_{n n}
\end{array}\right]
$$

Así, gracias al numeral 2.1.1, se puede colegir que:

$$
\left[Z_{n p}\right]=\left[Z_{p n}\right]^{T}
$$

Cabe anotar que, para el caso trifásico, cada una de estas submatrices es de dimensiones $3 \times 3$. Finalmente, se hace reducción de Kron con 2.1.1.

\section{Capacitancia:}

En el caso de las líneas subterráneas, la capacitancia se presenta entre cada conductor de fase y su cubrimiento externo.

En general, la capacitancia es directamente proporcional a la permitividad del medio $(\boldsymbol{\varepsilon})$ y a dimensiones geométricas; entre ellas, a una relación inversa a la separación entre conductores.

Es claro que para las líneas subterráneas la distancia entre conductores es muy pequeña con relación a la separación en las líneas aéreas; esta característica hace que la capacitancia sea mucho más grande en sistemas subterráneos.

Si se considera la teoría del electromagnetismo - campo eléctrico $(\boldsymbol{E})$, la Ley de Gauss, aplicada a un conductor infinito y cargado uniformemente, etc.-, en un sistema compuesto por varios conductores, se tiene que la diferencia de potencial entre el conductor $i$ y $j$ es: 


$$
v_{i j}=\frac{1}{2 \pi \varepsilon} \sum_{k=1}^{N} q_{k} \ln \frac{D_{k j}}{D_{k i}}
$$

Donde,

$D_{\mathbb{k}}=r_{k}: \quad$ radio del conductor $k$.

$D_{\sharp}: \quad$ distancia entre los conductores $k$ y $j$.

$D_{k}: \quad$ distancia entre los conductores $k$ e $i$.

$q_{k}: \quad$ carga del conductor $k$.

$N$ : número de conductores.

Esta ecuación entrega la diferencia de potencial entre dos puntos en el espacio dada la presencia de un número cualquiera de conductores cargados.

Ahora, se asume que, para el cable concéntrico, el campo eléctrico está confinado debido al apantallamiento ejercido por los hilos del neutro concéntrico. Este concepto tiene una incidencia especial en la estructura de la matriz que representa el efecto capacitivo (como se observará más adelante). En la FigurA NRO. 5 se ilustran los hilos concéntricos, así como algunos de los parámetros de utilidad en este modelamiento. En esa dirección, se define:

$R$ : radio del círculo que atraviesa el centro de los hilos concéntricos.

$D_{\mathrm{R}}: \quad$ distancia entre los hilos 1 y 2. 
$\theta_{\mathrm{L}}$ : ángulo entre los hilos 1 y 2 medido desde el centro de conductor.

$$
\theta_{12}=\frac{2 \pi}{k}
$$

Donde,

$k$ : número total de hilos del neutro.

$d_{c}: \quad$ diámetro del conductor de fase $=2 r_{c}$

$d_{s}: \quad$ diámetro de un hilo del conductor de neutro $=2 r_{s}$

Figura NRo. 5. Cable subterráneo con representación de hilos del neutro concéntrico.

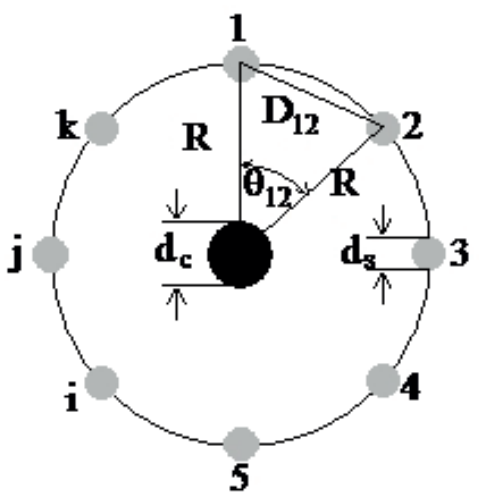

Si se considera que:

- La carga de cada hilo del neutro es $1 / \boldsymbol{k}$ de la carga en el conductor de fase y de signo opuesto.

- Todos los hilos del neutro están al mismo potencial y, por ende, la caída de potencial desde el conductor de fase hasta cada hilo es igual.

- Los hilos del neutro están puestos a tierra, entonces, esta caída de potencial se presenta entre el conductor y tierra. 
Entonces, es posible calcular la caída de potencial entre el conductor de fase y el hilo número 1 en presencia de los otros hilos empleando la expresión (2.16). Después de algunas manipulaciones algebraicas se obtiene lo siguiente:

$$
v_{p g}=\frac{q_{p}}{2 \pi \varepsilon}\left\{\ln \frac{R}{r_{c}}-\frac{1}{k}\left(\ln \frac{r_{s} k}{R}\right)\right\}
$$

donde $q_{p}$ es carga del conductor de fase.

La capacitancia entonces se encuentra como (2.19), a saber:

$$
C_{p g}=\frac{q_{p}}{v_{p g}}=\frac{2 \pi \varepsilon}{\ln \frac{R}{r_{c}}-\frac{1}{k}\left(\ln \frac{r_{s} k}{R}\right)} \quad \text { con } \varepsilon=\varepsilon_{0} \varepsilon_{r}
$$

Donde,

$$
\varepsilon_{0}=8.85 \times 10^{-9}\left[\frac{\mathrm{F}}{\mathrm{km}}\right]
$$

$\mathrm{Y}$

$\varepsilon_{r}: \quad$ permitividad del medio en que el campo eléctrico existe, es decir, del material de aislamiento del cable.

La admitancia es entonces:

$$
Y_{p g}=j \omega C_{p g}
$$

De este modo, para el efecto capacitivo, se tiene que la matriz de capacitancia shunt asociada, la cual se representa por medio de una matriz admitancia diagonal de $3 \times 3$ solo presenta elementos en la diagonal. Aquello que esté por fuera de ella es igual 
a cero debido al apantallamiento que posee cada cable, es decir, no existen efectos capacitivos mutuos, solo habrá capacitancias propias a tierra. El esquema es el siguiente:

Figura NRo. 6. Efecto capacitivo de la línea subterránea.

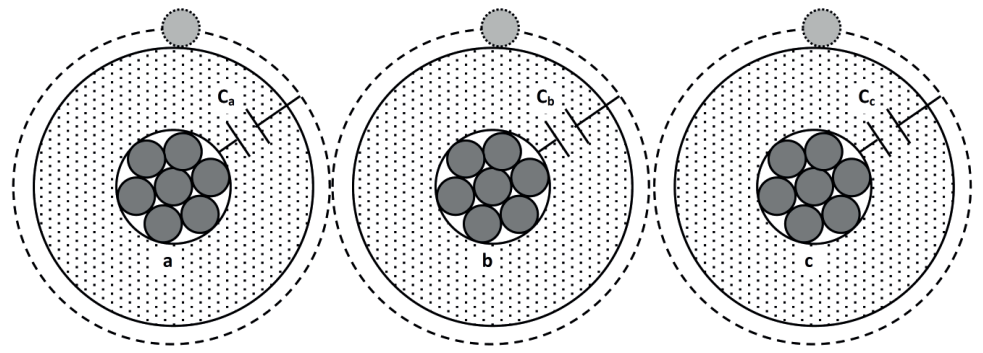

La matriz tiene la forma:

$$
C_{a b c}=\left[\begin{array}{ccc}
C_{a} & 0 & 0 \\
0 & C_{b} & 0 \\
0 & 0 & C_{c}
\end{array}\right]
$$

Si los tres cables son idénticos, entonces:

$$
C_{a}=C_{b}=C_{c}
$$

2.1.3.2 Cables con pantalla o cinta de cobre (semineutro).

Figura NRO. 7. Cables con pantalla o cinta de cobre.

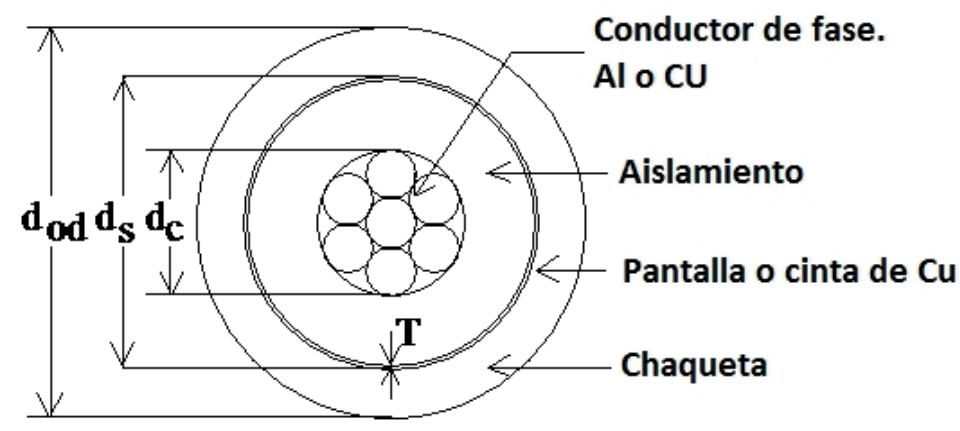


Usa cinta de cobre (neutro).

Para una corriente dada, la cinta es más barata que el neutro concéntrico (más fácil de construir). Estos son llamados «cables de potencia» pues se usan más en aplicaciones de gran corriente y en sistemas equilibrados, donde la corriente de desequilibrio es de baja magnitud y es soportada por la cinta. En el sistema de distribución, se utilizan estas cintas con contacto a tierra para fines de confinar el campo eléctrico, nunca o de manera poco común se instalan como neutro.

Los parámetros de estos cables son

$R$ : radio del círculo que atraviesa el centro de la cinta o pantalla, en $[\mathrm{m}]$.

$d_{c}$ : diámetro del conductor de fase, en [m].

$d_{o d}$ : diámetro exterior incluyendo la chaqueta, en $[\mathrm{m}]$.

$d_{s}$ : diámetro exterior de la cinta (incluyéndola).

T: espesor de la cinta cuyo valor típico en Colombia es 0.064 $\mathrm{mm}$. En USA, este valor es $5 \mathrm{mil}$ o $0.129 \mathrm{~mm}$.

$\rho$ : resistividad de la cinta, en $[\Omega-\mathrm{m}]$, a $50{ }^{\circ} \mathrm{C}$.

Con esta información se puede calcular la resistencia de la cinta.

$$
R_{\text {cinta }}=\frac{1000 \cdot \rho}{2 \cdot \pi \cdot R \cdot T}\left[\frac{\Omega}{\mathrm{km}}\right]
$$

donde $R$ y $T$ deberán estar en $[\mathrm{m}]$. 
En la cinta no se necesita corregir el flujo interno. El $R M G$ es exactamente el radio $R$ que pasa por el centro de la cinta.

$$
G M R_{\text {Cinta }}=R=\frac{d_{s}-T}{2}=\frac{(\text { Diametroexterior }-2 * \text { espesorchaqueta })-T}{2}
$$

Para el caso trifásico se numeran las fases con 1,2 y 3 ; y las cintas 4,5 y 6 .

Las distancias para el cálculo de las diferentes impedancias se manejan con los mismos criterios que en los cables con neutro de hilos.

\section{Capacitancia:}

Se toma la expresión para el caso de neutro de hilos. A saber:

$$
C_{p g}=\frac{q_{p}}{v_{p g}}=\frac{2 \pi \varepsilon}{\ln \frac{R}{r_{c}}-\frac{1}{k}\left(\ln \frac{r_{s} k}{R}\right)}
$$

Y simplemente, para la cinta, es equivalente a tener $\mathrm{k} \rightarrow \infty$. Es fácil de evaluar pues, al crecer $k$, el $\ln$ crece, pero menos que $1 / k$ que se hace pequeño. El segundo término del denominador tiende a 0 cuando $k$ se hace grande.

Evaluando el límite y multiplicando por $\omega$ para obtener la admitancia, se llega a:

$$
Y_{p g}=\frac{j 2 \pi \varepsilon \omega}{\ln \frac{R}{r_{c}}}
$$

Donde,

$$
\varepsilon=\varepsilon_{r} \varepsilon_{0}
$$


De esta índole, las unidades de la admitancia dependen del valor de $\varepsilon_{0}$.

$$
\varepsilon_{0}=8.85 \times 10^{-9} \frac{\mathrm{F}}{\mathrm{km}}=0.0142 \frac{\mu \mathrm{F}}{\mathrm{mile}}
$$

Si se emplea $\varepsilon_{o}$ en $[\mathrm{F} / \mathrm{km}]$, la admitancia dará en $[U / \mathrm{km}]$.

$\varepsilon_{r}$ es la permitividad relativa del medio en el cual el campo existe: el material aislante. $\mathrm{Al}$ respecto, se puede ver la TABLA NRO. 5 .

Tabla NRO. 5. Rangos de permitividad $\left(\varepsilon_{\mathrm{r}}\right)$.

\begin{tabular}{lc}
\hline \multicolumn{1}{c}{ Material } & Rango de permitividad $\left(\varepsilon_{r}\right)$ \\
\hline Polyvinyl Chloride (PVC) & $3.4-8.0$ \\
\hline $\begin{array}{l}\text { Ethylene-Propylene Rubber } \\
\text { (EPR) }\end{array}$ & $2.5-3.5$ \\
\hline Polyethylene (PE) & $2.5-2.6$ \\
\hline $\begin{array}{l}\text { Cross-linked Polyethlyene } \\
\text { (XLPE) }\end{array}$ & $2.3-6.0$ \\
\hline
\end{tabular}

A manera de ilustración, para un cable 1/0 XLPE, de cinta, monofásico, con un diámetro exterior de la cinta de 0.88 in; el espesor de la cinta es 5 mil (0.005 in). El diámetro del conductor de fase es 0.368 in $\left(r_{c}=0.184\right.$ in).

$$
\varepsilon=2.3 \varepsilon_{0}
$$

Según (2.24), el radio de un círculo que pasa a través del centro de la cinta es:

$$
R=0.4375 \text { inches }
$$

Y la admitancia según (2.26) es:

$$
Y_{p g}=j 55.66 \times 10^{-6}\left[\frac{U}{\mathrm{~km}}\right]
$$


En circuitos de $13.2 \mathrm{kV}$ y para un vano medio de $800 \mathrm{~m}$, $I_{\text {charging }}=0.1696 \mathrm{~A}$

Dado que puede tener alguna incidencia, se decide incluir el modelamiento del efecto capacitivo en tramos subterráneos.

\subsubsection{Conductores agrupados o en haz}

Para cables con dos, tres o cuatros conductores de una misma fase se aplica la teoría de conductores en haz, que consiste en reemplazarlo todos por uno solo equivalente ubicado en el centro de ellos y calculando el nuevo radio geométrico así:

Figura NRO. 8. Disposición geométrica de los conductores.
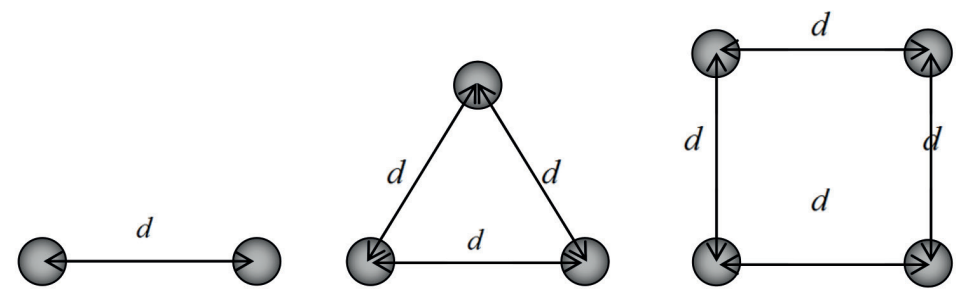

A continuación, se evidencia, en cada caso, el nuevo radio medio geométrico, en función del $R M G$ individual y de la distancia de separación $d$.

- Para $n=2$ o caso dúplex, es:

$$
R M G_{\text {new }}=\sqrt{R M G * d}
$$

- Para $n=3$ o caso tríplex, es:

$$
R M G_{\text {new }}=\sqrt[3]{R M G * d^{2}}
$$


- Para $n=4$ o caso cuádruplex, es:

$$
R M G_{\text {new }}=1.09 * \sqrt[4]{R M G * d^{3}}
$$

En el caso de cables subterráneos que van juntos, uno al lado del otro, $d$ corresponde al diámetro exterior de un cable XLPE.

La resistencia equivalente es igual a la resistencia de un conductor dividida entre 2 o 3 o 4, según sea dúplex, tríplex o cuádruplex.

\subsection{Modelamiento de cargas}

La complejidad de un modelo lo determina fundamentalmente el grado de exactitud deseado y el análisis que se esté realizando. La cantidad de estudios a realizar en un sistema eléctrico es supremamente variada, por citar algunos: confiabilidad, estabilidad, reconfiguración de redes, estudios de cortocircuito, flujos de carga, entre otros.

Adicionalmente, las cargas son muy diferentes entre sí de acuerdo con su naturaleza. Por ejemplo, las cargas industriales son muy diferentes a las residenciales, ellas pueden tener un alto porcentaje de motores con carga casi constante y un ciclo de operación definido; por el contrario, una carga residencial consta de electrodomésticos monofásicos funcionando de manera aleatoria.

Sin embargo, cuando se agregan las cargas existe cierto patrón de comportamiento del conjunto de cargas en un transformador de distribución. A nivel de subestaciones, este efecto promedio es más acentuado $y$, a nivel de líneas de transmisión, se alcanza una situación muy predecible. 
Nuestro interés se centra fundamentalmente en el modelamiento de las cargas que intervienen en el análisis de flujos de carga en sistemas de distribución secundaria, los cuales son, en gran medida, desequilibrados. En general, las cargas de los alimentadores de distribución pueden ser modeladas como conexiones $Y$ o $\Delta$; pueden estar conectadas a tres, dos o una fase con cualquier grado de desbalance.

Las cargas en los sistemas de distribución son generalmente especificadas por la potencia compleja que es consumida. Esta demanda puede ser especificada como potencia aparente en kilovoltiamperios (kVA) y factor de potencia, o potencia activa en kilovatios $(\mathrm{kW})$ y potencia reactiva en kilovoltiamperio reactivo (kVAr). La tensión especificada siempre será la tensión en los terminales de baja tensión de la subestación (transformador de distribución).

Los modelos de carga son usados en el proceso iterativo del estudio de flujo de potencia donde, primeramente, se suponen conocidas las tensiones de la carga. Todos los modelos son definidos, inicialmente, por una potencia compleja por fase y se asume una tensión línea-neutro para cargas conectadas en $Y$ o línea-línea para cargas conectadas en $\Delta$. Las unidades de la potencia compleja pueden ser voltiamperios (VA) y voltios (V), o voltiamperios (VA) por unidad (pu) y voltios (V) por unidad (pu). Para todas las cargas, se requieren las corrientes de línea que entran a la carga para el análisis de flujo de potencia.

Para las cargas conectadas en $Y$, la notación para la potencia compleja y la tensión especificada es:

Fase $i:\left|\mathrm{S}_{\mathrm{i}}\right| \angle \theta_{\mathrm{i}}=\mathrm{P}_{\mathrm{i}}+\mathrm{Q}_{\mathrm{i}} \quad \mathrm{y} \quad\left|\mathrm{V}_{\mathrm{in}}\right| \angle \delta_{\mathrm{i}}$

donde $i$ varía para cada una de las fases $i=a, b, c$. 
Para las cargas conectadas en $\Delta$, la notación para la potencia compleja y la tensión especificada es:

Fase $i:\left|\mathrm{S}_{\mathrm{i}}\right| \angle \theta_{\mathrm{i}}=\mathrm{P}_{\mathrm{i}}+\mathrm{Q}_{\mathrm{i}} \quad$ y $\left|\mathrm{V}_{\mathrm{i}}\right| \angle \delta_{\mathrm{i}}$

donde $i$ varía para cada pareja de fases $i=a b, b c, c a$.

FIGURA NRO. 9. Conexiones de la carga en Y y $\Delta$.
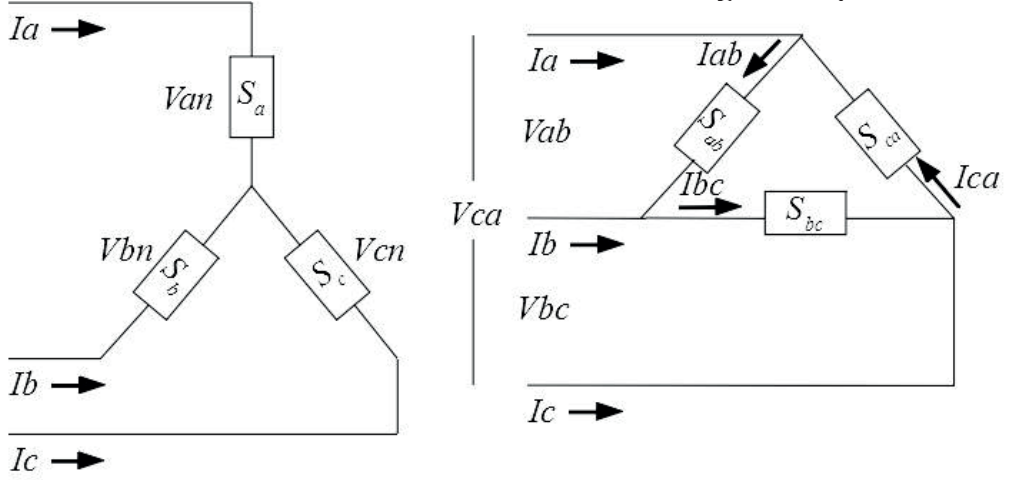

Las corrientes de línea entrando a una carga conectada en $\Delta$ son determinadas aplicando la primera ley de Kirchhoff en cada nodo. En forma matricial, las ecuaciones son:

$$
\left[\begin{array}{l}
\mathrm{I}_{\mathrm{a}} \\
\mathrm{I}_{\mathrm{b}} \\
\mathrm{I}_{\mathrm{c}}
\end{array}\right]=\left[\begin{array}{ccc}
1 & 0 & -1 \\
-1 & 1 & 0 \\
0 & -1 & 1
\end{array}\right]\left[\begin{array}{l}
\mathrm{I}_{\mathrm{ab}} \\
\mathrm{I}_{\mathrm{bc}} \\
\mathrm{I}_{\mathrm{ca}}
\end{array}\right]
$$

En el flujo de carga implementado, las cargas son modeladas como potencia activa y reactiva. Aquellas, al incluirse dentro del flujo de carga radial y dada la tensión, pueden entregar un valor de corriente de la siguiente manera:

$$
\begin{gathered}
\mathrm{S}_{\mathrm{i}}=\mathrm{S}_{\mathrm{n}} \\
\mathrm{I}_{\mathrm{i}}=\left(\frac{\mathrm{S}_{\mathrm{i}}}{\mathrm{V}_{\mathrm{i}}}\right)^{*}=\left(\frac{\mathrm{S}_{\mathrm{n}}}{\mathrm{V}_{\mathrm{i}}}\right)^{*}
\end{gathered}
$$


La expresión (2.33) debe ser interpretada adecuadamente para el caso en que la carga esté conectada en $Y$ o en $\Delta$, tal como se muestra en la TABLA NRO. 6.

TABLA NRO. 6. Corriente de carga según tipo de conexión (PQ constante).

\begin{tabular}{|c|c|}
\hline Conexión $Y$ & Conexión $\Delta$ \\
\hline $\mathrm{I}_{\mathrm{i}}=\left(\frac{\mathrm{S}_{\mathrm{i}}}{\mathrm{V}_{\text {in }}}\right)^{*}=\frac{\left|\mathrm{S}_{\mathrm{i}}\right|}{\left|\mathrm{V}_{\text {in }}\right|}\left|\delta_{\mathrm{i}}-\theta_{\mathrm{i}}=\right| \mathrm{I}_{\mathrm{i}}|| \alpha_{\mathrm{i}}$ & $\mathrm{I}_{\mathrm{i}}=\left(\frac{\mathrm{S}_{\mathrm{i}}}{\mathrm{V}_{\mathrm{i}}}\right)^{*}=\frac{\left|\mathrm{S}_{\mathrm{i}}\right|}{\left|\mathrm{V}_{\mathrm{i}}\right|}\left|\delta_{\mathrm{i}}-\theta_{\mathrm{i}}=\right| \mathrm{I}_{\mathrm{i}}|| \alpha_{\mathrm{i}}$ \\
\hline
\end{tabular}

donde $n$ es el neutro y el índice $i$ varía para cada una de las fases $(i=a, b, c)$ en la conexión en $Y$; y en la conexión en $\Delta$, varía para cada pareja de fases $(i=a b, b c, c a)$.

En sistemas secundarios de distribución, dado que hay neutro, todas las cargas se modelan en estrella $(Y)$. En el caso de cargas trifásicas, las cargas son $Y$ completas; y en el caso de cargas monofásicas, estas se modelan como $Y$ incompletas.

Las potencias activa, reactiva y aparente son expresadas por el fabricante para valores nominales de voltaje y frecuencia. Al variar estos valores, sus potencias se modifican; en especial, ante variaciones del voltaje en terminales del dispositivo. En un programa de flujo de carga, en cada iteración, se deberán recalcular las nuevas potencias de las cargas para los voltajes de dicha iteración.

Este comportamiento se ha modelado de diversas maneras; una de ellas ha consistido en expresar este en función de la relación del voltaje real al voltaje nominal, elevado a una potencia según se considere la carga como:

Potencia constante:

$$
S_{i}=S_{n}\left[\frac{V_{i}}{V_{n}}\right]^{0}
$$

Corriente constante:

$$
S_{i}=S_{n}\left[\frac{V_{i}}{V_{n}}\right]^{1}
$$


Impedancia constante: $\quad \mathrm{S}_{\mathrm{i}}=\mathrm{S}_{\mathrm{n}}\left[\frac{\mathrm{V}_{\mathrm{i}}}{\mathrm{V}_{\mathrm{n}}}\right]^{2}$

Donde, a valores nominales, $. S_{n}=P_{n}+Q_{n}$

A manera de ilustración, se muestra en la siguiente gráfica lo que le ocurre a $1 \mathrm{~kW}$ de carga cuando el voltaje en terminales de ella varía, utilizando las expresiones (2.34), (2.35) y (2.36).

FiguRA NRO. 10. Variación de la demanda ante cambios de voltaje para los diferentes modelamientos.

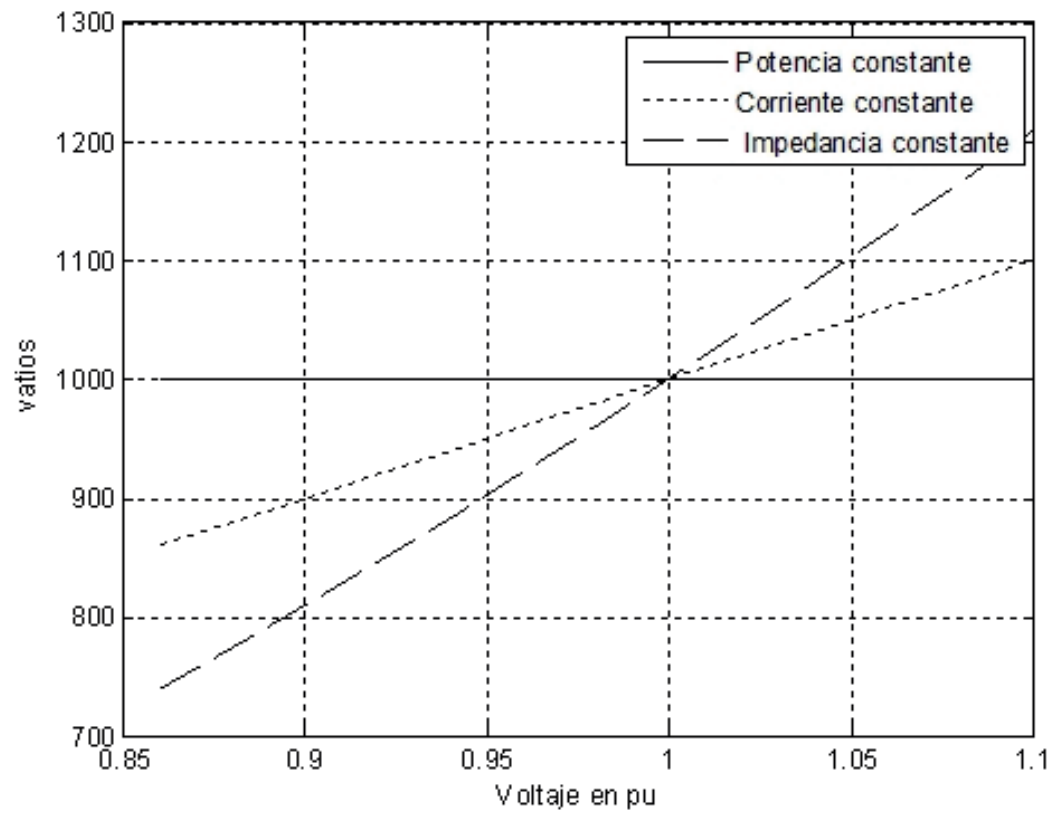

Se observa que, para un voltaje de 0.95 pu (regulación máxima en distribución), la demanda se reduce un $5 \%$, en el modelamiento de corriente constante y un $9 \%$, con impedancia constante. 


\subsubsection{Modelamiento como impedancia constant}

Este tipo de modelamiento corresponde generalmente a cargas de tipo residencial. Con,

$$
I_{n}=\frac{V_{n}}{Z_{n}}
$$

Si el voltaje real es $V_{i}$, entonces

$$
\begin{gathered}
I_{i}=\frac{V_{i}}{Z_{n}} \\
Z_{n}=\frac{V_{i}}{I_{i}}=\frac{V_{n}}{I_{n}} \\
I_{i}=I_{n}\left(\frac{V_{i}}{V_{n}}\right)
\end{gathered}
$$

Así,

$$
S_{i}=S_{n}\left[\frac{V_{i}}{V_{n}}\right]^{2}
$$

También,

$$
P_{i}=P_{n}\left[\frac{V_{i}}{V_{n}}\right]^{2}
$$

$\mathrm{Y}$

$$
Q_{i}=Q_{n}\left[\frac{V_{i}}{V_{n}}\right]^{2}
$$




\subsubsection{Modelamiento como corriente constante.}

Este tipo de modelamiento corresponde generalmente a cargas de tipo residencial. A saber,

$$
\begin{gathered}
I_{i}=\frac{V_{i}}{Z_{i}}=\frac{V_{n}}{Z_{n}} \\
Z_{i}=Z_{n}\left(\frac{V_{i}}{V_{n}}\right)
\end{gathered}
$$

La potencia aparente sería

$$
S_{i}=I_{i}{ }^{2} Z_{i}=I_{n}{ }^{2} Z_{n}\left(\frac{V_{i}}{V_{n}}\right)=S_{n}\left(\frac{V_{i}}{V_{n}}\right)
$$

Si el factor de potencia final es igual al original, el exponente se aplica a $P$ y a $Q$.

\subsubsection{Modelamiento como potencia constante: motores.}

Este tipo de modelamiento corresponde generalmente a cargas de motores,

$$
S_{i}=S_{n}
$$

dado que $S_{i}=S_{n}\left(\frac{V_{i}}{V_{n}}\right)^{0}$.

\subsubsection{Modelo Exponencial General}

Otra forma de modelar es hacerlo a través de un exponente general $n$ que pueda tomar diferentes valores. Con este se pretende modelar cualquier tipo de carga mixta. 


$$
S=S_{n}\left(\frac{V}{V_{n}}\right)^{x}
$$

Se puede establecer que, en sectores residenciales, el exponente general se estima que está entre 1 y 2 (intermedio entre impedancia y corriente constantes). Lo recomendable sería experimentalmente determinar el exponente adecuado para los sectores bajo estudio.

En [4] se plantea una expresión mucho más general aún, con exponentes diferentes para las potencias activas y para las reactivas del siguiente modo:

$$
P+\mathrm{j} Q=P_{n}\left(\frac{V}{V n}\right)^{k 1}+\mathrm{j} Q n\left(\frac{V}{V n}\right)^{k 2}
$$

Su utilización dependerá del conocimiento del sistema y de las herramientas computacionales que se posean.

\subsubsection{Modelo ZIP}

La tendencia actual es incluir en el modelo, con sus respectivos porcentajes, las componentes constantes de impedancia $(Z)$, de corriente $(I)$ y de potencia $(P)$. Modelamiento ZIP de la carga. Según [5]:

$$
P=P_{n}\left[\boldsymbol{a}_{p}\left(\frac{V}{V_{n}}\right)^{2}+\boldsymbol{b}_{p} \frac{V}{V_{n}}+\boldsymbol{c}_{p}\right]
$$

y

$$
Q=Q_{n}\left[\boldsymbol{a}_{q}\left(\frac{V}{V_{n}}\right)^{2}+\boldsymbol{b}_{q} \frac{V}{V_{n}}+\boldsymbol{c}_{q}\right]
$$


Donde los coeficientes $\boldsymbol{a}, \boldsymbol{b}$ y $\boldsymbol{c}$ de las ecuaciones (2.50) y (2.51) representan la porción de carga de impedancia constante, corriente constante y potencia constante, respectivamente. En nuestro caso, además, debido a la poca información los coeficientes: $a_{p}=a_{q} ; b_{p}=b_{q}$ y $c_{p}=c_{q}$. La tendencia también indica que el coeficiente $b=0$.

\subsubsection{Impedancia constante versus potencia constante}

A través de un ejemplo simple, se pueden observar algunas particularidades de ambos modelamientos. Una fuente $V_{s}$ alimenta, a través de una línea trifásica de $0.3+\mathrm{j} 0.4 \Omega$ por fase, una carga trifásica de $900 \mathrm{~kW}$ y factor de potencia 0.8 en atraso.

FIGURA NRO. 11. Circuito unifilar para modelar

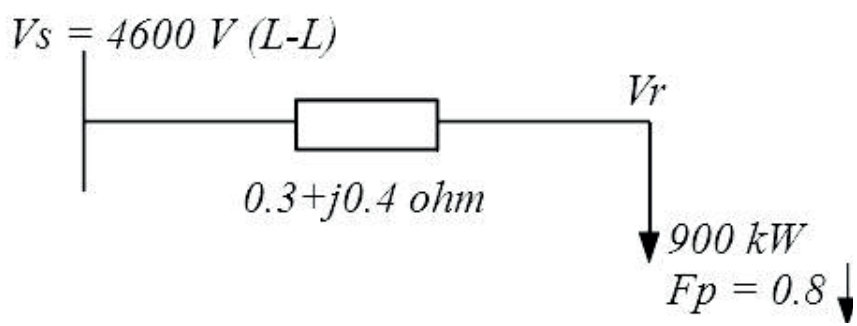

1) Este resuelve modelando la carga como impedancia constante para diferentes voltajes.

2) Se modela ahora la carga como de potencia constante. Su solución es un poco más dispendiosa. Dado que se conoce $V_{s}$ y la potencia en la carga, se deben hallar $V_{r}$ y la corriente para evaluar las pérdidas. Estese resuelve por fase y la solución se obtiene resolviendo la siguiente ecuación:

3)

$V_{r}^{4}+V_{r}^{2}\left[2(P R+Q X)-V_{s}^{2}\right]+S^{2} Z^{2}=0$ 
Donde,

$Z: \quad$ magnitud de la impedancia del conductor.

$R, X: \quad$ componente resistiva y reactiva de la impedancia del conductor

$S: \quad$ potencia aparente por fase de la carga.

$P, Q: \quad$ potencia activa y reactiva por fase de la carga.

FiguRA NRO. 12. Pérdidas de potencia en vatios para el ejemplo.

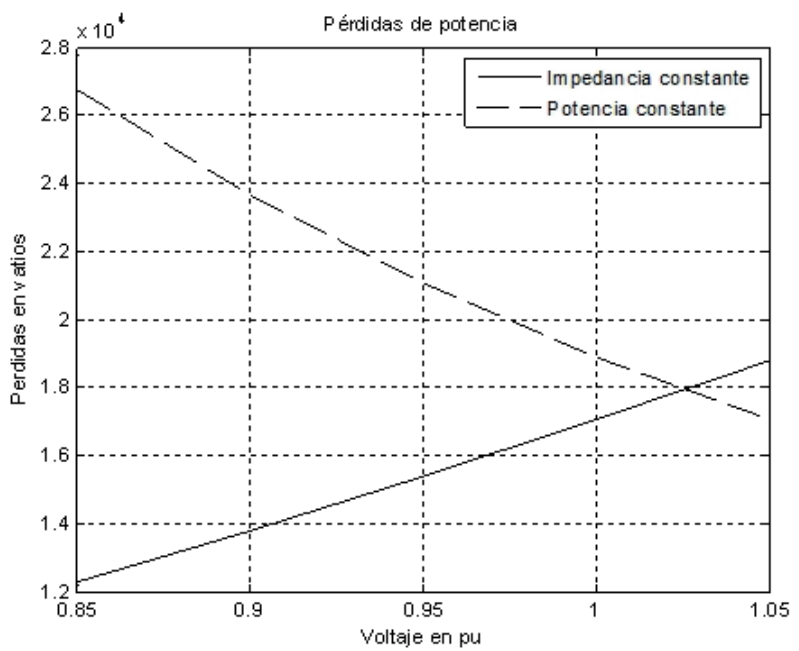

Figura NRo. 13. Porcentaje de pérdidas respecto de la fuente.

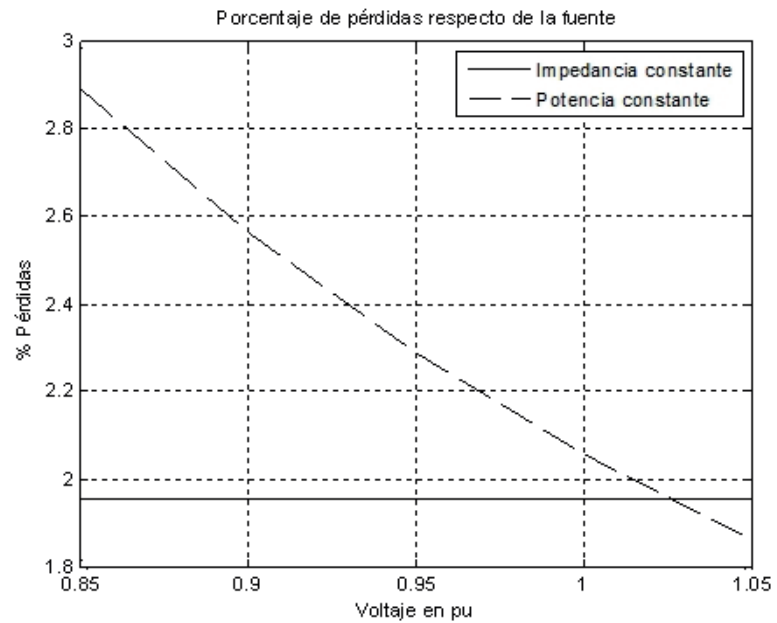


De las anteriores figuras se concluye lo siguiente:

1) Modelar a potencia constante arroja más pérdidas tanto en vatios como en porcentaje y, en especial, ante caídas de voltaje como es lo normal en redes secundarias.

2) Modelar a impedancia constante arroja que, ante reducciones de la tensión, las pérdidas disminuyen, pero en porcentaje respecto de la fuente son constantes; este porcentaje es insensible ante cambios de voltaje.

3) Nota. En la Figura NRO. 13 se puede observar que cuando el voltaje de la fuente es de $0.95 \mathrm{pu}$, las pérdidas en sus respectivas bases son de $1.95 \%$ para $\mathrm{Z}$ constante y de $2.3 \%$ para potencia constante. En este caso, la diferencia es de $0.35 \%$, la cual se incrementa para redes de pobre regulación de voltaje.

En redes secundarias, los estudios analíticos y programas digitales se pueden simplificar omitiendo la categoría de corriente constante (la más rara de los tres tipos) y usando solamente cargas de tipo impedancia constante y potencia constante, o el modelo ZIP. Este modelo ZIP es quizás el más recomendado pues recoge en uno solo los modelos más utilizados como los de impedancia constante y el de potencia constante dejando también la posibilidad de incluir algún efecto de corriente constante.

Se debe tener en cuenta que, al momento de la escribir este texto, según requerimiento de [1], las cargas se deben modelar como de impedancia constante.

\subsection{Modelamiento de transformadores}

Las pérdidas en un transformador pueden ser de potencia reactiva y potencia activa. En esta sección, nos ocuparemos fundamentalmente de las pérdidas activas, las cuales se evalúan en vacío y con carga. 


\subsubsection{Pérdidas en vacío}

Las pérdidas de potencia en vacío son iguales a las pérdidas nominales en vacío $\left(P_{N o}\right)$, las cuales corresponden aproximadamente a las pérdidas del hierro.

$$
P_{N o}=P_{f e}
$$

Puesto que estas pérdidas son - por propósitos prácticos - constantes y dadas por el fabricante, en un año, las pérdidas de energía en vacío $\left(P_{E o}\right)$ serán:

$$
P_{E o}=P_{N o} \times 8760[\mathrm{kWh}]
$$

Y, en un día,

$$
P_{E o}=P_{N o} \times 24[\mathrm{kWh}]
$$

\subsubsection{Pérdidas con carga}

Las pérdidas de potencia con carga $\left(P_{P c}\right)$ son básicamente $I^{2} R$ de los devanados. La pérdida de potencia con carga nominal $P_{P c n}$ (dadas por el fabricante) son:

Para el transformador trifásico:

$$
P_{P c n}=3 \times I_{n}^{2} \times R=3\left(\frac{k V A_{n}}{\sqrt{3} \times k V}\right)^{2} \times R
$$

Y, en general, a cualquier otra demanda:

$$
P_{P c}=3 \times I^{2} \times R=3\left(\frac{k V A}{\sqrt{3} \times k V}\right)^{2} \times R
$$


Así, si se divide (2.57) entre (2.56), se obtiene la expresión (2.58). Esta es general para transformadores monofásicos o trifásicos, a saber:

$$
\frac{P_{P c}}{P_{P c n}}=\left(\frac{k V A}{k V A_{n}}\right)^{2}
$$

Para cualquier estado de carga del transformador, las pérdidas con carga (en $\mathrm{kW}$ ) se pueden evaluar fácilmente con:

$$
P_{P c}=P_{P c n}\left(\frac{k V A}{k V A_{n}}\right)^{2}
$$

Donde,

kVA: demanda de la carga, en [kVA].

$k V A_{n}: \quad$ valor nominal del transformador, en [kVA].

$P_{P c n}: \quad$ pérdida de potencia a carga nominal (dada por el fabricante en $\mathrm{kW}$ ).

El cociente $\frac{k V A}{k V A_{n}}$ es una especie de índice de carga del transformador el cual, para demanda máxima del transformador, recibe el nombre de factor de utilización $(f u)$.

$$
f u=\frac{k V A_{\max }}{k V A_{n}}
$$

La pérdida de potencia se puede evaluar para cualquier estado de carga; sin embargo, es de interés evaluarlas en la condición de máxima demanda. Así, la ecuación (2.60) toma la forma de:

$$
P_{P c \max }=P_{P c n} \times f u^{2}
$$


Se debe recordar que:

$$
\text { Pérdidas de energía cu }=F P \times P_{P c n} \times(f u)^{2} \times T
$$

Donde el FP es el factor de pérdidas.

Si se reemplaza (2.61) en (2.62), se obtienen las pérdidas en carga (en el cobre):

$$
\text { Pérdidas de energía } c u=F P \times P_{P c n} \times(f u)^{2} \times T
$$

\subsubsection{Pérdidas Totales}

Las pérdidas de potencia en el pico son:

$$
\text { Pérdidas de potencia pico }=P_{f e}+P_{P c n} \times(f u)^{2}
$$

Las pérdidas totales de energía son:

Pérdidas de energía totales $=P_{f e} \times T+F P \times P_{P c n} \times(f u)^{2} \times T(2.65)$

En caso de transformadores monofásicos, se utiliza [6], [7] y en el caso de transformadores trifásicos, [8], [9]. De estas normas, se obtienen las pérdidas en el hierro $\left(P_{f e}\right)$ y las pérdidas en el cobre a carga nominal $\left(P_{p c}\right)$. Estos valores se expresan en vatios.

Para un día, las pérdidas en [kWh] se calculan así:

$$
\text { PETRA }=\left(P f e * 24+\sum_{i=1}^{24} P p c *\left\{\frac{I_{i}}{I_{n}}\right\}^{2}\right) / 1000
$$


Donde,

$I_{i}: \quad$ corriente que entrega el transformador en la hora $i$.

$I_{n}: \quad$ corriente nominal del secundario del transformador.

Para los transformadores sin carga (transformadores energizados cuya carga en el secundario es mínima o nula), únicamente se calculan las pérdidas en el hierro del transformador.

\subsection{Modelamiento de medidores}

Los medidores de energía están compuestos por un par de bobinas para cada fase; por lo tanto, los medidores monofásicos, bifásicos y trifásicos poseen, respectivamente, 1 par, 2 pares y 3 pares de bobinas. Cada elemento medidor está constituido por una bobina de potencial y una bobina de corriente, de las cuales, la pérdida en la bobina de potencial es asumida por el operador de red. Esta pérdida no varía a lo largo del día, ya que está sometida a una tensión prácticamente constante la cual permite asumir que la pérdida de energía en el medidor es independiente de la carga.

Para calcular la pérdida de energía en un medidor de energía, se necesita conocer el valor de pérdidas por elemento medidor y la cantidad total de medidores monofásicos, bifásicos y trifásicos.

Por lo tanto, las pérdidas de potencia activa $\mathrm{Pm}$ correspondientes a los medidores de energía de un circuito de distribución, se calculan como:

$$
P m=\sum_{i=1}^{N} W m_{i}
$$


Donde,

$N$ : número de medidores.

$W m_{i}$ : consumo de potencia según el tipo de medidor.

Para conocer el consumo de potencia de los medidores existen dos opciones: hacer un análisis del consumo de los medidores utilizados según su información técnica, o utilizar los consumos máximos permitidos por las normas técnicas colombianas [10], [11], [12] y [13] del ICONTEC (Instituto Colombiano de Normas Técnicas).

\subsubsection{Consumo de los medidores electromecánicos y electrónicos según su información técnica}

A continuación, se presenta un ejemplo de la información técnica de medidores usualmente utilizados por empresas de comercialización de energía eléctrica en Colombia. Para los medidores presentados, se muestra el consumo de cada uno, clasificándolo por tecnologías (electromecánicos y/o estáticos) y tipo de conexión (número de fases).

En las siguientes tablas, se puede observar el consumo de cada referencia y el respectivo promedio para cada tipo de conexión, dependiendo de la tecnología que implementen.

TABLA NRO. 7. Consumo de medidores electromecánicos para conexión directa de acuerdo con su tipo de conexión.

\begin{tabular}{|c|c|c|c|c|c|c|c|c|c|}
\hline \multicolumn{10}{|c|}{ Medidores electromecánicos para conexión directa } \\
\hline \multicolumn{10}{|c|}{ Monofásicos Bifilares } \\
\hline \multirow{2}{*}{ Marca } & \multirow{2}{*}{ Tipo de Energía } & \multirow{2}{*}{ Tipo } & \multirow{2}{*}{ Referencia } & \multirow{2}{*}{$\mathrm{F}[\mathrm{Hz}]$} & \multirow{2}{*}{$\mathrm{T}[\mathrm{V}]$} & \multicolumn{2}{|c|}{$\mathrm{I}[\mathrm{A}]$} & \multicolumn{2}{|c|}{ Consumo } \\
\hline & & & & & & $\mathrm{Ib}(\mathrm{min})$ & $\mathrm{Ib}(\max )$ & {$[\mathrm{W}]$} & [VA] \\
\hline CHINT & Activa & $\begin{array}{c}\text { Inducción } \\
\text { Monofásicos } \\
\text { Bifilares }\end{array}$ & DD701 & 60 & 120 & 10 & 60 & 1 & 5 \\
\hline Chint & Activa & $\begin{array}{l}\text { Inducción } \\
\text { Monofásicos } \\
\text { Trifilares }\end{array}$ & DD701P & 60 & $120 / 240$ & 10 & 60 & & \\
\hline Holley & Activa & $\begin{array}{c}\text { Inducción } \\
\text { Monofásicos } \\
\text { Bifilares }\end{array}$ & HLD03 & 60 & $120 / 240$ & 10 & 60 & 1 & 5 \\
\hline Holley & Activa & $\begin{array}{c}\text { Inducción } \\
\text { Monofásicos } \\
\text { Trifilares }\end{array}$ & HLD03-II & 60 & $120 / 240$ & 10 & 60 & 1 & 5 \\
\hline
\end{tabular}




\begin{tabular}{|c|c|c|c|c|c|c|c|c|c|}
\hline Tecun & Activa & $\begin{array}{c}\text { Monofásicos } \\
\text { Bifilares }\end{array}$ & DDS994 & 60 & 120 & 5 & 60 & 1 & 6 \\
\hline \multicolumn{8}{|c|}{ Promedio } & 1 & 5.25 \\
\hline \multicolumn{10}{|c|}{ Trifásicos Tetrafilares } \\
\hline \multirow{2}{*}{ Marca } & \multirow{2}{*}{ Tipo de Energía } & \multirow{2}{*}{ Tipo } & \multirow{2}{*}{ Referencia } & \multirow{2}{*}{$\mathrm{F}[\mathrm{Hz}]$} & \multirow{2}{*}{$\mathrm{T}[\mathrm{V}]$} & \multicolumn{2}{|c|}{$\mathrm{I}[\mathrm{A}]$} & \multicolumn{2}{|c|}{ Consumo } \\
\hline & & & & & & $\mathrm{Ib}(\mathrm{min})$ & $\mathrm{Ib}(\max )$ & {$[\mathrm{W}]$} & {$[\mathrm{VA}]$} \\
\hline Holley & Activa & $\begin{array}{c}\text { Inducción } \\
\text { Trifásicos } \\
\text { Tetrafilares }\end{array}$ & DT58CT & 60 & $3 * 120 / 208$ & 5 & 10 & 1 & 6 \\
\hline Holley & Activa & $\begin{array}{l}\text { Inducción } \\
\text { Trifásicos } \\
\text { Tetrafilares }\end{array}$ & DT58 & 60 & $3 * 120 / 208$ & 20 & 100 & 1 & 6 \\
\hline \multicolumn{8}{|c|}{ Promedio } & 1 & 6 \\
\hline
\end{tabular}

TABLA NRO. 8. Consumo de medidores estáticos monofásicos bifilares para conexión directa.

\begin{tabular}{|c|c|c|c|c|c|c|c|c|c|}
\hline \multicolumn{10}{|c|}{ Medidores estáticos para conexión directa } \\
\hline \multicolumn{10}{|c|}{ Monofásicos Bifilares } \\
\hline \multirow[b]{2}{*}{ Marca } & \multirow[b]{2}{*}{$\begin{array}{l}\text { Tipo de } \\
\text { Energía }\end{array}$} & \multirow[b]{2}{*}{ Tipo } & \multirow[b]{2}{*}{ Referencia } & \multirow[b]{2}{*}{$\begin{array}{c}\mathrm{F}[\mathrm{H} \\
\mathrm{z}]\end{array}$} & \multirow[b]{2}{*}{$\mathrm{T}[\mathrm{V}]$} & \multicolumn{2}{|c|}{$\mathrm{I}[\mathrm{A}]$} & \multicolumn{2}{|c|}{ Consumo } \\
\hline & & & & & & $\mathrm{Ib}(\min )$ & $\begin{array}{l}\mathrm{Ib} \\
(\max )\end{array}$ & [W] & $\begin{array}{l}{[\mathrm{V}} \\
\mathrm{A}]\end{array}$ \\
\hline Inhemeter & Activa & $\begin{array}{c}\text { Electrónicos } \\
\text { Monofásicos } \\
\text { Trifilares }\end{array}$ & DDZ1513 & 60 & $120 / 240$ & 5 & 80 & 0.8 & 4.5 \\
\hline Hexing & Activa & $\begin{array}{c}\text { Estáticos } \\
\text { Monofásicos } \\
\text { Bifilares }\end{array}$ & HXE12R & 60 & 120 & 5 & 100 & 1 & 10 \\
\hline Huyu & Activa & $\begin{array}{c}\text { Estáticos } \\
\text { Monofásicos } \\
\text { Bifilares }\end{array}$ & DDS881 & 60 & 120 & 5 & 60 & 1 & 6 \\
\hline Linyang & Activa & $\begin{array}{l}\text { Electrónicos } \\
\text { Monofásicos } \\
\text { Bifilares }\end{array}$ & $\begin{array}{l}\text { LY-BM11 - } \\
\text { LY-BM12 }\end{array}$ & 60 & 120 & 5 & 60 & 1 & 10 \\
\hline $\begin{array}{l}\text { Applied } \\
\text { Meters }\end{array}$ & Activa & $\begin{array}{c}\text { Electrónicos } \\
\text { Monofásicos } \\
\text { Bifilares } \\
\end{array}$ & $\begin{array}{c}\text { AMS B1B- } \\
\text { OA1 }\end{array}$ & 60 & 120 & 5 & 60 & 0.4 & 7.5 \\
\hline $\begin{array}{l}\text { Applied } \\
\text { Meters }\end{array}$ & Activa & $\begin{array}{c}\text { Electrónicos } \\
\text { Monofásicos } \\
\text { Trifilares }\end{array}$ & $\begin{array}{l}\text { AMS B1B- } \\
\text { OA3 }\end{array}$ & 60 & $120 / 240$ & 5 & 60 & 0.4 & 7.5 \\
\hline CHINT & Activa & $\begin{array}{c}\text { Electrónicos } \\
\text { monofásicos } \\
\text { Bifilares }\end{array}$ & DDS666 & 60 & 120 & 10 & 100 & 0.4 & 4 \\
\hline CHINT & Activa & $\begin{array}{c}\text { Electrónicos } \\
\text { Monofásicos } \\
\text { Trifilares }\end{array}$ & DDS666P & 60 & $120 / 240$ & 10 & 100 & 0.1 & 2.6 \\
\hline Elster & Activa & $\begin{array}{c}\text { Estáticos } \\
\text { Monofásicos } \\
\text { Bifilares }\end{array}$ & $\begin{array}{c}\text { SC-MR } \\
\text { (Escorpión } \\
\text { Registrador } \\
\text { Mecánico) o } \\
\text { SC-LCD } \\
\text { (Registrados } \\
\text { LCD) }\end{array}$ & 60 & 120 & 5 & 60 & 0.5 & 6 \\
\hline Elster & Activa & $\begin{array}{c}\text { Estáticos } \\
\text { Monofásicos } \\
\text { Bifilares }\end{array}$ & $\begin{array}{c}\text { SC-MR } \\
\text { (Escorpión } \\
\text { Registrador } \\
\text { Mecánico) o } \\
\text { SC-LCD } \\
\text { (Registrados } \\
\text { LCD) }\end{array}$ & 60 & 120 & 5 & 100 & 0.5 & 6 \\
\hline HOLLEY & Activa & $\begin{array}{c}\text { Electrónicos } \\
\text { Monofásicos } \\
\text { Bifilares }\end{array}$ & DDS28-II & 60 & 120 & 10 & 80 & 1 & 10 \\
\hline HOLLEY & Activa & $\begin{array}{c}\text { Electrónicos } \\
\text { Monofásicos } \\
\text { Bifilares }\end{array}$ & DDS28-I & 60 & 120 & 10 & 80 & 1 & 10 \\
\hline
\end{tabular}




\begin{tabular}{|c|c|c|c|c|c|c|c|c|c|}
\hline Mega IP & Activa & $\begin{array}{c}\text { Electrónico } \\
\text { Monofäsico } \\
\text { Bifilar }\end{array}$ & SGM71 & 60 & 120 & 10 & 100 & & \\
\hline Israel & Activa & $\begin{array}{c}\text { Estático } \\
\text { Monofásico } \\
\text { Trifilar }\end{array}$ & DSS-8888 & 60 & $120 / 240$ & 5 & 60 & 2 & 10 \\
\hline Mega IP & Activa & $\begin{array}{c}\text { Electrónico } \\
\text { Monofásico } \\
\text { Bifilar }\end{array}$ & $\begin{array}{c}\text { SGM71-60 } \\
\text { SGM71L- } \\
60\end{array}$ & 60 & 120 & 5 & 60 & 1 & 5 \\
\hline \multicolumn{70}{|c|}{ Promedio } \\
\hline
\end{tabular}

TABLA NRO. 9. Consumo de medidores estáticos bifásicos bifilares para conexión directa.

\begin{tabular}{|c|c|c|c|c|c|c|c|c|c|}
\hline \multicolumn{10}{|c|}{ Medidores estáticos para conexión directa } \\
\hline \multicolumn{10}{|c|}{ Bifásicos Bifilares } \\
\hline \multirow[b]{2}{*}{ Marca } & \multirow{2}{*}{$\begin{array}{l}\text { Tipo de } \\
\text { Energía }\end{array}$} & \multirow[b]{2}{*}{ Tipo } & \multirow[b]{2}{*}{ Referencia } & \multirow{2}{*}{$\begin{array}{c}\mathrm{F}[\mathrm{H} \\
\mathrm{z}]\end{array}$} & \multirow[b]{2}{*}{$\mathrm{T}[\mathrm{V}]$} & \multicolumn{2}{|c|}{$\mathrm{I}[\mathrm{A}]$} & \multicolumn{2}{|c|}{ Consumo } \\
\hline & & & & & & $\mathrm{Ib}(\min )$ & $\begin{array}{c}\mathrm{Ib} \\
(\max )\end{array}$ & {$[\mathrm{W}]$} & $\begin{array}{c}\text { [VA } \\
]\end{array}$ \\
\hline $\begin{array}{l}\text { Applied } \\
\text { Meters }\end{array}$ & Activa & $\begin{array}{c}\text { Electrónicos } \\
\text { Bifásicos } \\
\text { Trifilares } \\
\end{array}$ & $\begin{array}{c}\text { AMT B1E- } \\
\text { OR2T }\end{array}$ & 60 & $2 * 120 / 208$ & 5 & 100 & 0.4 & 1 \\
\hline $\begin{array}{l}\text { Applied } \\
\text { Meters }\end{array}$ & Activa & $\begin{array}{c}\text { Electrónicos } \\
\text { Bifásicos } \\
\text { Trifilares } \\
\end{array}$ & $\begin{array}{c}\text { AMT B1A- } \\
\text { OA2T }\end{array}$ & 60 & $2 * 120 / 208$ & 10 & 100 & 0.4 & 1 \\
\hline Elster & Activa & $\begin{array}{c}\text { Electrónicos } \\
\text { Bifásicos } \\
\text { Trifilares }\end{array}$ & A 1200 & 60 & $\begin{array}{c}2 * 120 / 208 \\
\ldots 2 * 277 / 4 \\
80 \\
\end{array}$ & 5 & 100 & 2 & 10 \\
\hline Elster & Activa & $\begin{array}{c}\text { Electrónico } \\
\text { Bifásico } \\
\text { Bifilar }\end{array}$ & A1100 & 60 & $2 * 120 / 208$ & 5 & 100 & 0.9 & 9 \\
\hline Elster & $\begin{array}{l}\text { Activa - } \\
\text { Reactiva }\end{array}$ & $\begin{array}{l}\text { Estáticos, } \\
\text { Bifásicos } \\
\text { Trifilares y } \\
\text { Trifásicos } \\
\text { Tetrafilares }\end{array}$ & A 1052 & 60 & $\begin{array}{c}2 * 120 / 208 \\
\ldots 2 * 277 / 4 \\
80 \\
3 * 120 / 208 \\
\ldots \\
3 * 277 / 480 \\
\end{array}$ & $1 / 5$ & 10 & 0.66 & 9.1 \\
\hline Estrom & Activa & $\begin{array}{c}\text { Electrónico } \\
\text { Trifásico } \\
\text { Tetrafilar }\end{array}$ & DTS353 & 60 & $2 * 120 / 208$ & 5 & 100 & 1 & 7 \\
\hline Génesis II & $\begin{array}{l}\text { Activa - } \\
\text { Reactiva }\end{array}$ & $\begin{array}{c}\text { Electrónico } \\
\text { Bifásico } \\
\text { Trifilar }\end{array}$ & EBM23 & 60 & $2 * 120 / 208$ & 5 & 100 & 0.3 & 2.6 \\
\hline Israel & Activa & $\begin{array}{c}\text { Estático } \\
\text { Bifásico } \\
\text { Trifilar }\end{array}$ & DSS- 8888 & 60 & $2 * 120 / 208$ & 5 & 100 & 2 & 10 \\
\hline Itron/Actaris & Activa & $\begin{array}{c}\text { Electrónicos, } \\
\text { Bifásicos } \\
\text { Trifilar } \\
\end{array}$ & ACE 3000 & 60 & $\begin{array}{l}2 * 120 / 220 \\
2 * 120 / 208\end{array}$ & 5 & 100 & 1 & 2.5 \\
\hline Itron/Actaris & Activa & $\begin{array}{c}\text { Electrónicos } \\
\text { Bifásicos } \\
\text { Trifilares } \\
\end{array}$ & ACE 3000 & 60 & $\begin{array}{l}2 * 127 / 220 \\
2 * 120 / 208\end{array}$ & 5 & 100 & 1 & 2.5 \\
\hline Tecun & Activa & $\begin{array}{c}\text { Estáticos } \\
\text { Bifásico } \\
\text { Trifilar } \\
\end{array}$ & DSS949 & 60 & $2 * 120 / 208$ & 5 & 100 & 1 & 10 \\
\hline & & & Promedio & & & & & 1 & 5.88 \\
\hline
\end{tabular}


TABLA NRO. 10. Consumo de medidores estáticos trifásicos tetrafilares para conexión directa.

\begin{tabular}{|c|c|c|c|c|c|c|c|c|c|}
\hline \multicolumn{10}{|c|}{ Medidores estáticos para conexión directa } \\
\hline \multicolumn{10}{|c|}{ Trifásicos Tetrafilares } \\
\hline \multirow[b]{2}{*}{ Marca } & \multirow[b]{2}{*}{$\begin{array}{l}\text { Tipo de } \\
\text { Energía }\end{array}$} & \multirow[b]{2}{*}{ Tipo } & \multirow[b]{2}{*}{ Referencia } & \multirow[b]{2}{*}{$\begin{array}{c}\mathrm{F}[\mathrm{H} \\
\mathrm{z}]\end{array}$} & \multirow[b]{2}{*}{$\mathrm{T}[\mathrm{V}]$} & \multicolumn{2}{|c|}{$\mathrm{I}[\mathrm{A}]$} & \multicolumn{2}{|c|}{ Consumo } \\
\hline & & & & & & $\begin{array}{c}\mathrm{Ib} \\
\text { (Min) }\end{array}$ & $\begin{array}{c}\mathrm{Ib} \\
(\max )\end{array}$ & $\begin{array}{c}{[\mathrm{W}} \\
]\end{array}$ & $\begin{array}{c}{[\mathrm{VA}} \\
]\end{array}$ \\
\hline Hexing & Activa & $\begin{array}{c}\text { Estáticos } \\
\text { Trifásicos } \\
\text { Tetrafilares }\end{array}$ & HXE34K & 60 & $\begin{array}{c}2 * 120 / 2 * 20 \\
8\end{array}$ & 5 & 100 & 0.6 & 0.6 \\
\hline $\begin{array}{l}\text { Applied } \\
\text { Meters }\end{array}$ & Activa & $\begin{array}{c}\text { Electrónicos } \\
\text { Trifásicos } \\
\text { Tetrafilares }\end{array}$ & $\begin{array}{l}\text { AMT B1E- } \\
\text { OR4T }\end{array}$ & 60 & $3 * 120 / 208$ & 5 & 100 & 0.4 & 1 \\
\hline Elgama & $\begin{array}{l}\text { Activa - } \\
\text { Reactiva }\end{array}$ & $\begin{array}{c}\text { Estáticos } \\
\text { Trifásicos } \\
\text { Tetrafilares }\end{array}$ & GAMA 300 & 60 & $\begin{array}{c}3 * 57,7 / 100 \\
\ldots \\
277 / 480\end{array}$ & 5 & 120 & 0.5 & 1 \\
\hline Elster & $\begin{array}{l}\text { Activa - } \\
\text { Reactiva }\end{array}$ & $\begin{array}{c}\text { Electrónicos } \\
\text { Trifásicos } \\
\text { Tetrafilares }\end{array}$ & $\mathrm{A} 1800$ & 60 & $\begin{array}{c}* 57,7 / 100 \\
\ldots \\
277 / 480\end{array}$ & 5 & 120 & 3 & 4.4 \\
\hline Elster & $\begin{array}{l}\text { Activa - } \\
\text { Reactiva }\end{array}$ & $\begin{array}{c}\text { Electrónicos } \\
\text { Trifásicos } \\
\text { Tetrafilares }\end{array}$ & A1350 & 60 & $3 * 120 / 208$ & 5 & 100 & 3 & 4.4 \\
\hline Elster & Activa & $\begin{array}{c}\text { Electrónico } \\
\text { Trifásico } \\
\text { Tetrafilar } \\
\end{array}$ & A 1100 & 60 & $3 * 120 / 208$ & 5 & 100 & 0.9 & 9 \\
\hline Elster & $\begin{array}{l}\text { Activa - } \\
\text { Reactiva }\end{array}$ & $\begin{array}{l}\text { Estáticos, } \\
\text { Trifásicos } \\
\text { Tetrafilares }\end{array}$ & A1052 & 60 & $\begin{array}{c}3 * 120 / 208 \\
\ldots \\
3 * 277 / 480\end{array}$ & 5 & 120 & $\begin{array}{c}0.6 \\
6\end{array}$ & 9.1 \\
\hline Génesis II & $\begin{array}{l}\text { Activa - } \\
\text { Reactiva }\end{array}$ & $\begin{array}{l}\text { Electrónico } \\
\text { Trifásico } \\
\text { Tetrafilar }\end{array}$ & ETM34 & 60 & $3 * 120 / 208$ & 5 & 100 & 0.2 & 2.8 \\
\hline Iskra & $\begin{array}{l}\text { Activa - } \\
\text { Reactiva }\end{array}$ & $\begin{array}{c}\text { Electrónico } \\
\text { Trifásico } \\
\text { Tetrafilar } \\
\end{array}$ & MT-174 & 60 & $3 * 120 / 208$ & 5 & 120 & 0.6 & 10 \\
\hline Itron/Actaris & Activa & $\begin{array}{c}\text { Electrónicos } \\
\text { Trifásicos } \\
\text { Tetrafilares }\end{array}$ & ACE 3000 & 60 & $\begin{array}{l}3 * 120 / 220 \\
3 * 120 / 208\end{array}$ & 10 & 100 & 1 & 2.5 \\
\hline Itron/Actaris & Activa & $\begin{array}{l}\text { Trifásico } \\
\text { Tetrafilar }\end{array}$ & ACE 3000 & 60 & $\begin{array}{l}2 * 127 / 220 \\
2 * 120 / 208\end{array}$ & 5 & 100 & 0.4 & 3 \\
\hline Inhemeter & Activa & $\begin{array}{c}\text { Estáticos } \\
\text { Trifásicos } \\
\text { Tetrafilares }\end{array}$ & DTZ1513 & 60 & $3 * 120 / 208$ & 5 & 100 & 1.5 & 4 \\
\hline Landis GYR & $\begin{array}{l}\text { Activa - } \\
\text { Reactiva }\end{array}$ & $\begin{array}{c}\text { Estático } \\
\text { Trifásico } \\
\text { Tetrafilar }\end{array}$ & ZMG 310 & 60 & $\begin{array}{c}* 110190 \\
\ldots \\
3 * 277 / 480\end{array}$ & 5 & 120 & \begin{tabular}{|l}
0.8 \\
\end{tabular} & 5 \\
\hline Tecun & Activa & $\begin{array}{c}\text { Estáticos } \\
\text { Trifásicos } \\
\text { Tetrafilares } \\
\end{array}$ & DTS949 & 60 & $3 * 120 / 208$ & 5 & 100 & 2 & 10 \\
\hline Tecun & Activa & $\begin{array}{c}\text { Estáticos } \\
\text { Trifásicos } \\
\text { Tetrafilares }\end{array}$ & DST949 & 60 & $3 * 120 / 208$ & 5 & 100 & 2 & 10 \\
\hline & & & Promedio & & & & & 1.6 & 5.55 \\
\hline
\end{tabular}


TABLA NRO. 11. Consumo de medidores estáticos trifásicos tetrafilares para conexión semidirecta

\begin{tabular}{|c|c|c|c|c|c|c|c|c|c|}
\hline \multicolumn{10}{|c|}{ Medidores estáticos para conexión semidirecta e indirecta } \\
\hline \multicolumn{10}{|c|}{ Trifásicos Tetrafilares } \\
\hline \multirow{2}{*}{ Marca } & \multirow{2}{*}{$\begin{array}{l}\text { Tipo de } \\
\text { Energía }\end{array}$} & \multirow{2}{*}{ Tipo } & \multirow{2}{*}{ Referencia } & \multirow{2}{*}{$\begin{array}{c}\mathrm{F}[\mathrm{Hz} \\
]\end{array}$} & \multirow{2}{*}{$\mathrm{T}[\mathrm{V}]$} & \multicolumn{2}{|c|}{$\mathrm{I}[\mathrm{A}]$} & \multicolumn{2}{|c|}{ Consumo } \\
\hline & & & & & & $\mathrm{Ib}(\mathrm{min})$ & $\mathrm{Ib}(\max )$ & [W] & {$[\mathrm{VA}]$} \\
\hline Elgama & $\begin{array}{l}\text { Activa - } \\
\text { Reactiva }\end{array}$ & $\begin{array}{c}\text { Estáticos } \\
\text { Trifásicos } \\
\text { Tetrafilares }\end{array}$ & GAMA 300 & 60 & $\begin{array}{c}3 * 57,7 / 100 \\
\ldots \\
277 / 480 \\
\end{array}$ & 1 & 10 & 0.5 & 1 \\
\hline $\begin{array}{c}\text { Elgama- } \\
\text { Elektronika }\end{array}$ & $\begin{array}{l}\text { Activa - } \\
\text { Reactiva }\end{array}$ & $\begin{array}{c}\text { Estáticos } \\
\text { Trifásicos } \\
\text { Tetrafilares }\end{array}$ & EPQS & 60 & $\begin{array}{c}3 * 57,7 / 100 \\
\ldots \\
277 / 480\end{array}$ & 1 & 10 & 0.5 & 1 \\
\hline $\begin{array}{l}\text { Elgama- } \\
\text { Elektronika }\end{array}$ & $\begin{array}{l}\text { Activa - } \\
\text { Reactiva }\end{array}$ & $\begin{array}{c}\text { Estáticos } \\
\text { Trifásicos } \\
\text { Tetrafilares } \\
\end{array}$ & EPQS & 60 & $\begin{array}{c}* 57,7 / 100 \\
\ldots \\
254 / 440\end{array}$ & $1-1.2$ & $5 / 6$ & 0.5 & 1 \\
\hline Elster & $\begin{array}{l}\text { Activa - } \\
\text { Reactiva }\end{array}$ & $\begin{array}{c}\text { Estáticos } \\
\text { Trifásicos } \\
\text { Tetrafilares } \\
\end{array}$ & AS 1440 & 60 & $3 * 120 / 208$ & 1 & 6 & 0.7 & 0.8 \\
\hline Elster & Activa & $\begin{array}{c}\text { Electrónico } \\
\text { Trifásico } \\
\text { Tetrafilar }\end{array}$ & A1200 & 60 & $\begin{array}{c}3 * 120 / 208 \\
\ldots \\
3 * 277 / 480 \\
\end{array}$ & 5 & 6 & 2 & 10 \\
\hline Elster & $\begin{array}{l}\text { Activa - } \\
\text { Reactiva }\end{array}$ & $\begin{array}{c}\text { Electronicos } \\
\text { Multifuncio } \\
\text { nales, } \\
\text { Trifásicos } \\
\text { Tetrafilares }\end{array}$ & A1800 & 60 & $\begin{array}{c}3 * 57,7 / 100 \\
\ldots \\
277 / 480\end{array}$ & 1 & 10 & 3 & - \\
\hline Elster & $\begin{array}{l}\text { Activa - } \\
\text { Reactiva }\end{array}$ & $\begin{array}{c}\text { Electronicos } \\
\text { Multifuncio } \\
\text { nales, } \\
\text { Trifásicos } \\
\text { Tetrafilares }\end{array}$ & A1800 & 60 & $\begin{array}{c}3 * 57,7 / 100 \\
\ldots \\
277 / 480\end{array}$ & 1 & 10 & 3 & - \\
\hline Elster & $\begin{array}{l}\text { Activa - } \\
\text { Reactiva }\end{array}$ & $\begin{array}{c}\text { Estáticos } \\
\text { Trifásicos } \\
\text { Tetrafilares } \\
\end{array}$ & A1350 & 60 & $3 * 120 / 208$ & 5 & 6 & 1.5 & 2.2 \\
\hline Elster & Activa & $\begin{array}{c}\text { Electrónico } \\
\text { Trifásico } \\
\text { Tetrafilar } \\
\end{array}$ & A1100 & 60 & $3 * 120 / 208$ & 1 & 10 & 0.9 & 9 \\
\hline EMH & $\begin{array}{l}\text { Activa - } \\
\text { Reactiva }\end{array}$ & $\begin{array}{c}\text { Electrónico } \\
\text { Trifásico } \\
\text { Tetrafilar }\end{array}$ & LZQJ-XC & 60 & $\begin{array}{c}3 * 57,7 / 100 \\
\ldots \\
3 * 240 / 415\end{array}$ & 1 & 10 & 0.8 & 1.2 \\
\hline Inhemeter & $\begin{array}{l}\text { Activa - } \\
\text { Reactiva }\end{array}$ & $\begin{array}{c}\text { Estáticos } \\
\text { Trifásicos } \\
\text { Tetrafilares }\end{array}$ & DTZ1513 & 60 & $3 * 120 / 208$ & 1 & 10 & 1.5 & 4 \\
\hline Iskra & $\begin{array}{l}\text { Activa - } \\
\text { Reactiva }\end{array}$ & $\begin{array}{c}\text { Electrónico } \\
\text { Trifásico } \\
\text { Tetrafilar }\end{array}$ & MT-174 & 60 & $3 * 120 / 208$ & 1 & 6 & 0.6 & 10 \\
\hline Landis GYR & Activa & $\begin{array}{c}\text { Electrónicos } \\
\text { Trifásicos } \\
\text { Tetrafilares } \\
\end{array}$ & ZMD405 & 60 & $\begin{array}{c}3 * 58 / 100 \\
\ldots \\
3 * 240 / 415 \\
\end{array}$ & 1 & 10 & 0.8 & 3.6 \\
\hline Landis GYR & $\begin{array}{l}\text { Activa - } \\
\text { Reactiva }\end{array}$ & $\begin{array}{c}\text { Electrónicos } \\
\text { Trifásicos } \\
\text { Tetrafilares }\end{array}$ & ZMG 405 & 60 & $\begin{array}{c}3 * 58 / 100 \\
\ldots \\
3 * 240 / 415\end{array}$ & 5 & 10 & 0.8 & 5 \\
\hline Tecun & $\begin{array}{l}\text { Activa - } \\
\text { Reactiva }\end{array}$ & $\begin{array}{c}\text { Estáticos } \\
\text { Trifásicos } \\
\text { Tetrafilares } \\
\end{array}$ & DTS949 & 60 & $3 * 120 / 208$ & 1.5 & 6 & 2 & 10 \\
\hline Tecun & $\begin{array}{l}\text { Activa - } \\
\text { Reactiva }\end{array}$ & $\begin{array}{c}\text { Estáticos } \\
\text { Trifásicos } \\
\text { Tetrafilares }\end{array}$ & DTS 949 & 60 & $3 * 120 / 208$ & 5 & 10 & 2 & 10 \\
\hline Tecun & $\begin{array}{l}\text { Activa - } \\
\text { Reactiva }\end{array}$ & $\begin{array}{c}\text { Estáticos } \\
\text { Trifásicos } \\
\text { Tetrafirares }\end{array}$ & DST949 & 60 & $3 * 120 / 208$ & 1.5 & 6 & 2 & 10 \\
\hline & & & Promedio & & & & & 1.4 & 5.37 \\
\hline
\end{tabular}




\subsubsection{Consumo de los medidores electromecánicos y electrónicos según las normas NTC 2147, NTC 22882147 y NTC 4052}

De acuerdo con [11], los medidores electromecánicos deben tener un consumo máximo de potencia entre $2 \mathrm{~W}$ y $3 \mathrm{~W}$. Para los medidores estáticos el consumo estipulado, sin importar la clase de precisión y el tipo de conexión, es de $2 \mathrm{~W}$ según las normas [10] y [12], las cuales toman como referencia la norma IEC-62053-21. La TABLA NRO. 12 presenta un resumen de esta información.

TABLA NRO. 12. Consumo de potencia en circuitos de tensión según las NTC correspondientes.

\begin{tabular}{|c|c|c|c|c|c|}
\hline & Clase & $\begin{array}{l}\text { NTC } 2147 \\
\text { Medidores } \\
\text { estáticos }\end{array}$ & $\begin{array}{c}\text { NTC } 2288 \\
\text { Medidores } \\
\text { electromecánicos }\end{array}$ & $\begin{array}{c}\text { NTC } 4052 \\
\text { Medidores } \\
\text { estáticos }\end{array}$ & $\begin{array}{c}\text { NTC } 4649 \\
\text { Equipo para } \\
\text { medidores de } \\
\text { energía }\end{array}$ \\
\hline \multirow{4}{*}{$\begin{array}{c}\text { Fuente de alimentación } \\
\text { conectada al circuito de tensión }\end{array}$} & $0.2 \mathrm{~S}$ & $2 \mathrm{~W}$ & ------ & ------ & ------ \\
\hline & $0.5 \mathrm{~S}$ & $2 \mathrm{~W}$ & ------ & ------ & ------ \\
\hline & 1 & ------ & ------ & $2 W$ & ------ \\
\hline & 2 & ------ & ------ & $2 \mathrm{~W}$ & ------ \\
\hline \multirow{4}{*}{$\begin{array}{c}\text { Fuente de alimentación no } \\
\text { conectada al circuito de tensión }\end{array}$} & $0.2 \mathrm{~S}$ & $0.5 \mathrm{VA}$ & ----- & ----- & ----- \\
\hline & $0.5 \mathrm{~S}$ & $0.5 \mathrm{VA}$ & ------ & ------ & ------ \\
\hline & 1 & ----- & ------ & $0.5 \mathrm{VA}$ & ------ \\
\hline & 2 & ------ & ----- & $0.5 \mathrm{VA}$ & ----- \\
\hline \multirow{3}{*}{ Monofásicos y polifásicos } & 0.5 & ------ & $3 \mathrm{~W}$ & ------ & ------ \\
\hline & 1 & ----- & $3 \mathrm{~W}$ & ----- & ----- \\
\hline & 2 & ----- & $2 \mathrm{~W}$ & ----- & ------ \\
\hline \multirow{3}{*}{ Multienergía } & $1 \varphi$ & ------ & ------ & ------ & $3.0 \mathrm{~W}$ \\
\hline & $2 \varphi$ & -.--- & ----- & ------ & $2.5 \mathrm{~W}$ \\
\hline & $3 \varphi$ & ------ & ------ & ------ & $2.0 \mathrm{~W}$ \\
\hline \multirow{3}{*}{ Multifunción } & $1 \varphi$ & ----- & ------ & ------ & $5.0 \mathrm{~W}$ \\
\hline & $2 \varphi$ & ------ & ------ & ------ & $3.5 \mathrm{~W}$ \\
\hline & $3 \varphi$ & ----- & ---.-- & ------ & $3.0 \mathrm{~W}$ \\
\hline
\end{tabular}

\subsubsection{Análisis comparativo de los consumos de los} medidores a partir de su información técnica y las normas nacionales

La Tabla NRo. 13 presenta un resumen del comportamiento de los consumos consultados en catálogos comerciales y los máximos permitidos por la norma. 
TABLA NRO. 13. Resumen del consumo promedio para todos los medidores versus consumo estipulado por la norma.

\begin{tabular}{|l|l|c|c|}
\cline { 3 - 4 } \multicolumn{2}{c|}{} & \multicolumn{2}{c|}{ Consumo Promedio [W] } \\
\hline \multirow{2}{*}{ Tipo de medidores } & \multirow{2}{*}{ Cantidad de hilos } & $\begin{array}{c}\text { Catálogo de } \\
\text { Medidores }\end{array}$ & Norma \\
\hline \multirow{2}{*}{ Electromecánicos para conexión directa } & Monofásicos bifilares & 1 & {$[2-3]$} \\
\cline { 3 - 4 } & Trifásicos tetrafilares & 1 & {$[2-3]$} \\
\hline \multirow{3}{*}{ Estáticos para conexión directa } & Monofásicos bifilares & 1.1 & 2 \\
\cline { 3 - 4 } & Bifásicos bifilares & 1 & 2 \\
\cline { 2 - 4 } & Trifásicos tetrafilares & 1.6 & 2 \\
\hline Estáticos para conexión semidirecta e indirecta & Trifásicos tetrafilares & 1.4 & 2 \\
\hline
\end{tabular}

Observando la información consignada en la TABLA NRO. 7, el consumo promedio para medidores electromecánicos, tanto para los monofásicos como los trifásicos, es de $1 \mathrm{~W}$. Comparando este valor con lo establecido en [11] (es decir, 2.5 W), se evidencia que los medidores comerciales presentan un consumo menor.

Observando la información consignada desde la TABLA NRO. 8 hasta la TABLA NRO. 11, el consumo promedio máximo para medidores estáticos monofásicos, bifásicos y trifásicos es de 1.6 W. Comparando este promedio con lo establecido en [10] y [12] (es decir, $2 \mathrm{~W}$ ), se verifica que los medidores comerciales presentan un consumo menor.

Es aconsejable que cada operador de red investigue el valor medio de pérdidas de energía por elemento medidor a través de ensayos debido a que el valor de pérdidas de potencia activa en este elemento puede variar dependiendo del tipo de medidor usado. De este modo, este puede influir de forma significativa en los resultados de pérdidas técnicas del sistema.

\subsection{Modelamiento de acometidas}

El modelamiento de las acometidas y, en consecuencia, el cálculo de las pérdidas en las acometidas depende del grado de información que se posea, tal como: 
- El consumo detallado de energía de cada usuario y su ubicación (nodo de conexión dentro del circuito), calibre de acometida y longitud.

- La información de cada nodo. Normalmente, solo hay información de cuántos usuarios hay, más no de cuáles.

- Los circuitos sin red.

La herramienta computacional desarrollada para calcular las pérdidas en nivel de tensión 1 aplica el primer caso, donde se posee información completa.

\subsubsection{Redes con información detallada}

Este es el mejor escenario porque las redes de distribución secundaria tienen para cada usuario su facturación, número de hilos, calibre y longitud de la acometida. Aquí, el flujo de carga determina el valor de la carga de cada usuario y realiza el cálculo de pérdidas con los datos reales de corriente y parámetros del conductor. Este es el caso que aplica para la herramienta desarrollada.

\subsubsection{Redes donde solo se conoce la cantidad de usuarios por poste}

Para los casos en que solo se conoce cuántos usuarios hay por poste, pero no se tiene información del tipo de servicio, calibre de acometida, longitud de acometida y facturación anual; una manera de modelar las acometidas puede ser utilizando la curva de demanda máxima diversificada y estimando la demanda para el grupo de usuarios conectados a dicho nodo. Se considera una longitud promedio de acometida, un calibre típico y se evalúa la impedancia equivalente $(R)$ del grupo de usuarios. Con la carga obtenida del nodo, se evalúan las pérdidas en estas acometidas. 


\subsubsection{Circuitos que no tienen red}

Para el caso de circuitos de distribución secundaria que no tienen red, se obtiene la carga pico en [kVA], se divide entre el número de fases y se obtiene la corriente pico. Se asume una longitud promedio y un calibre típico. Luego, se evalúa la impedancia equivalente del grupo de usuarios. En este orden de ideas, las pérdidas a corriente pico $\left(P_{\text {pico }}\right)$ serán,

$I^{2} \times(3 \times R)$ : si el número de fases es 3 o 2 en un sistema trifásico.

$$
\begin{aligned}
I^{2} \times(2 \times R): & \text { en los casos restantes }(1 \text { fase de sistema monofásico; } \\
& 1 \text { fase de sistema trifásico o } 2 \text { fases de sistema } \\
& \text { monofásico }) .
\end{aligned}
$$

En un día, las pérdidas se atenúan con el factor de pérdidas $(F P)$, expresado en $[\mathrm{kWh}]$. Este se obtiene de la curva de carga:

$$
P E A C O=\left(P_{\text {pico }} * 24 * F P\right) / 1000
$$

Donde $P_{\text {pico }}$ son las pérdidas en las acometidas a corriente pico. 


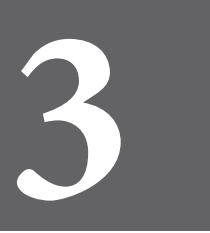

CAPÍTULO

TRES 


\section{Metodología para el cálculo de pérdidas técnicas de redes radiales a partir de flujo de carga de barrido iterativo}

En este capítulo, se presenta una metodología de flujo de carga para el cálculo de pérdidas técnicas en sistemas de distribución secundarios. La metodología considera un modelo trifásico del sistema (Figura Nro. 14) y utiliza, como información primaria, la curva de carga del transformador; es decir, tal y como ocurre en los sistemas de distribución reales, se parte del desconocimiento de la potencia consumida por la carga (usuarios), a través de la curva de carga del transformador, y de la facturación de energía de los usuarios. De esta índole, se realiza un proceso de barrido iterativo que permita estimar la potencia consumida por los usuarios y la potencia activa perdida en la red. La suma entre la potencia consumida por los usuarios y las pérdidas de potencia activa en la red debe coincidir con la curva de carga vista en el transformador.

\subsection{Fundamentos de la metodología}

Figura NRo. 14. Modelo trifásico de la red secundaria radial.

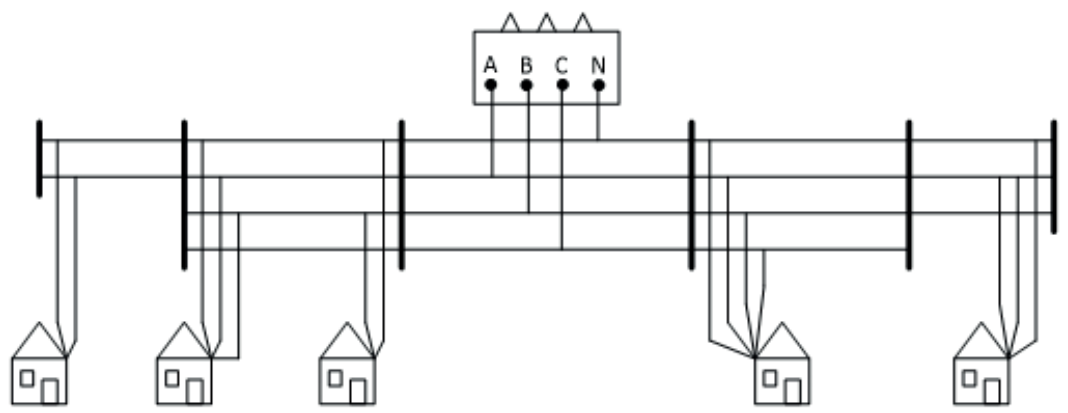


La metodología desarrollada considera un modelo trifásico del sistema, tal como se muestra en la FigURA NRO. 14, e incorpora aspectos operativos reales como los siguientes:

- El desbalance del circuito en faseo y tipo de conexión de los usuarios (bifilar, trifilar, tetrafilar), es decir, el desbalance en potencia de la red de distribución.

- Las características distintivas de cada usuario: tipo de conexión, longitud de acometida, tipo de medidor y consumo de energía.

- La curva de carga diaria del transformador (24 horas), suministrada por el flujo de carga en el nivel de tensión 2, y discriminada para un día típico ordinario y un día típico festivo.

- La energía consumida por las luminarias conectadas al circuito secundario.

Así, para el modelamiento, se considera una red trifásica tetrafilar o monofásica trifilar, según sea el caso. Dicho de otro modo, no se consideran equivalentes monofásicos. Los tipos de red considerados son:

- Aéreas o subterráneas.

- Abiertas o trenzadas.

- Con conductores desnudos o aislados.

- Con conductores de cobre o aluminio.

Se considera un máximo de 3 fases por tramo con 1 neutro en sistemas trifásicos, y 2 fases por tramo con 1 neutro en sistemas monofásicos. Aunque los tramos pueden contener conductores en paralelo, es decir, varios conductores por fase. 
Para cada hora de un día típico (ordinario y festivo), se realiza un flujo de carga para obtener las pérdidas de potencia activa y verificar las condiciones operativas de cargabilidad de red y regulación de tensión. Con las 24 horas evaluadas, se obtienen las pérdidas técnicas de energía para el día típico ordinario y el día típico festivo. Las pérdidas de energía para el día ordinario y el día festivo se multiplican por la cantidad de días ordinarios y festivos que tenga el año con el fin de obtener las pérdidas de energía anual.

Los resultados de las pérdidas de energía anual se presentan en relación con el circuito completo y sus componentes (transformador, red, acometidas y contadores).

\subsection{Flujo de carga}

Las redes de distribución presentan características muy particulares como topologías radiales, múltiples conexiones, cargas de distinta naturaleza, líneas con resistencia mayor a la reactancia, líneas sin transposiciones, y combinación de líneas aéreas y subterráneas.

En el análisis de flujo de potencia de un sistema de distribución, se conoce la tensión en la fuente (subestación o transformadores de distribución), la impedancia de cada tramo de red, la potencia compleja de todas las cargas y el modelo de cada una de las cargas (potencia constante, impedancia constante, corriente constante o una combinación de las anteriores).

El análisis de flujo de potencia de un sistema de distribución determina la magnitud de las tensiones y los ángulos en todos los nodos del alimentador. Usando estos resultados es posible calcular, entre otros, los siguientes valores:

- Flujo de potencia en cada sección de línea, corrientes (magnitud y ángulo) y factor de potencia. 
- Pérdidas de potencia en cada sección de línea.

- Potencia total inyectada al alimentador.

El flujo de carga empleado está basado en el método de barrido iterativo. Este método supone un ordenamiento de las barras del sistema de acuerdo con lo que se presenta en 0 . Una vez la red ha sido ordenada, el método de barrido iterativo se realiza en dos etapas consecutivas. La primera consiste en aplicar la primera ley de Kirchhoff en cada nodo. Se inicia desde los terminales hasta llegar a la subestación (barrido hacia arriba). Para esto, es necesario suponer un perfil de tensión inicial. La segunda etapa consiste en aplicar la segunda ley de Kirchhoff para obtener las caídas de tensión en cada línea. Esta comienza en la subestación hasta llegar a los nodos terminales (barrido hacia abajo). Para ello, se utilizan los datos de corrientes encontrados en la primera etapa.

Este proceso permite obtener nuevos valores para las tensiones nodales. La realización de las dos etapas anteriores constituye una iteración del proceso global. El método converge cuando la diferencia de pérdidas activas entre dos iteraciones sucesivas es menor que un error establecido.

Debido a que los sistemas de distribución no son balanceados, es necesario que el estudio de flujo de potencia considere el modelamiento trifásico de los diferentes componentes de la red. El flujo de carga para sistemas de distribución desbalanceados permite el adecuado estudio de problemas más complejos como balance de cargas, estudios de caída de tensión, compensación capacitiva y reconfiguración, entre otros. 


\subsection{Procedimiento}

Figura NRo. 15. Diagrama de flujo de la metodología propuesta.

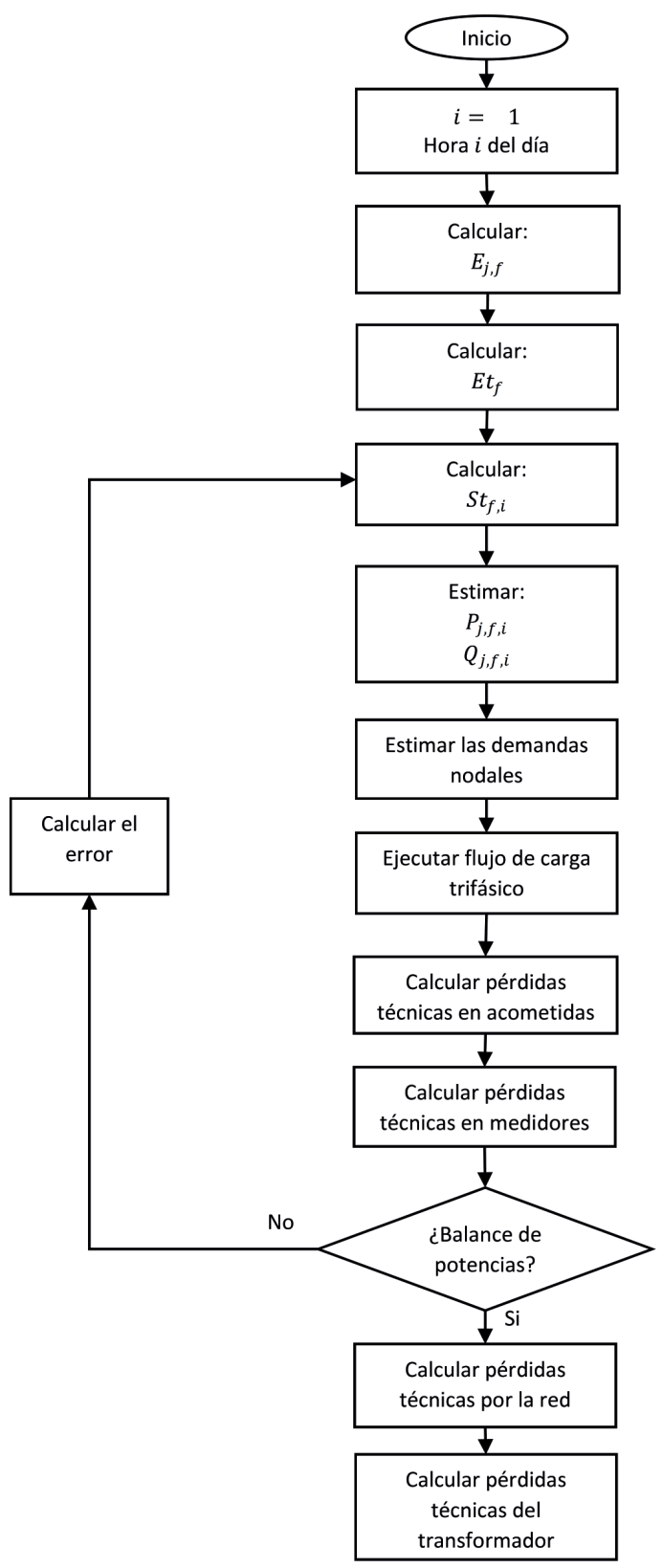


Las pérdidas se calculan según el procedimiento presentado en el diagrama de flujo de la Figura NRO. 15, así:

a. Se calcula la energía consumida por fase de cada usuario $j\left(E_{j, f}\right)$. Se asumen condiciones balanceadas según el tipo de conexión. Es decir, para un usuario trifilar (F-F-N) se reparte la energía consumida entre las dos fases.

b. Se calcula la energía total consumida por fase $\left(E t_{f}\right)$ del circuito secundario, según la energía consumida por fase de cada usuario. La sumatoria se realiza por fase, teniendo en cuenta los usuarios conectados a cada una de estas.

$$
E t_{f}=\sum_{\substack{j=1 \\ U \in f}}^{n u} E_{j, f}
$$

Donde,

nu: $\quad$ número de usuarios.

$f: \quad$ indica las fases del sistema $(A, B, C$ caso trifásico o $A, B$ para el caso monofásico).

c

De acuerdo con la potencia entregada a la hora $i$ del día $\left(S_{i}\right)$, se calcula la potencia total entregada en cada fase por el transformador.

$$
S t_{f, i}=S_{i} \cdot \frac{E t_{f}}{\sum_{f=1}^{N f} E t_{f}}
$$

Donde,

Nf: $\quad$ indica la cantidad de fases del circuito. 
d Se estima la demanda por fase de cada usuario para la hora $i\left(S_{j_{i}}\right)$, mediante la siguiente expresión:

$$
S_{j, f, i}=S t_{f, i} \cdot \frac{E_{j, f}}{E t_{f}}
$$

e $\quad$ Se estima la potencia activa y reactiva consumida por cada usuario en la hora $i\left(P_{j, f, i}\right.$ y $\left.Q_{j, f, i}\right)$.

$$
\begin{aligned}
& P_{j, f, i}=S_{j, f, i} \cdot f p_{i} \\
& Q_{j, f, i}=S_{j, f, i} \cdot \operatorname{sen}\left(\cos ^{-1}\left(f p_{i}\right)\right)
\end{aligned}
$$

Donde,

$f p_{i}: \quad$ indica el factor de potencia en la hora $i$

f. Se suman las potencias de los usuarios conectados a un mismo nodo para obtener la potencia nodal del sistema.

g. Con los datos de demanda estimados en el numeral anterior, se ejecuta un flujo de carga desbalanceado y se obtienen las tensiones $\left(V c_{f, i}\right)$ y corrientes $\left(I c_{f, i}\right)$ por fase del sistema.

h. Se calcula la potencia por fase, entregada por el transformador $\left(S c_{f, i}\right)$.

$$
S c_{f, i}=V c_{f, i} \cdot / c_{f, i}{ }^{*}
$$

i. Con las tensiones nodales, se calcula la corriente en cada fase consumida por cada usuario y se calculan las pérdidas por los conductores de la acometida $\left(\operatorname{Saco}_{j, i}\right)$.

j. Se calculan pérdidas de los medidores $\left(\right.$ Smed $\left._{j, i}\right)$ considerando los límites de pérdidas establecidos en [10], [11], [12] y [13] del ICONTEC.

k. Se verifica el balance de potencia de acuerdo con la siguiente expresión: 


$$
\sum_{f=1}^{n f} S c_{f, i}+\sum_{j=1}^{n u} S a c o_{j, i}+\sum_{j=1}^{n u} \operatorname{Smed}_{j, i}=\sum_{f=1}^{n f} S t_{f, i}
$$

1. Si la diferencia es menor a $1 \mathrm{~W}$, el proceso se detiene; de lo contrario, se modifica el valor $S_{i}$ según la diferencia obtenida en el literal anterior, y se vuelve al paso c.

Una vez el proceso ha convergido, se calculan las pérdidas técnicas en los tramos de red secundaria y las pérdidas en el transformador con los datos de corrientes por fase del sistema, de acuerdo con la siguiente expresión:

$$
P_{\text {energía }}=P_{f e} \times T+F P \times P_{p c n} \times f u^{2} \times T
$$

Donde,

FP: $\quad$ factor de pérdidas.

fu: factor de utilización.

$P_{f e}$ y $P_{p c n}$ se toman de [6] y [8] del ICONTEC.

Después de realizar el cálculo de pérdidas técnicas para un circuito, se determina cuál fue, durante las 24 horas del análisis, el tramo de red secundaria con mayor cargabilidad y el nodo con mayor caída de tensión. La máxima cargabilidad de la red se expresa en porcentaje de su límite térmico; la máxima caída de tensión en el circuito se expresa en porcentaje de tensión nominal de la siguiente forma:

$$
\% \Delta V=\left[\frac{\mathrm{V}_{\text {nom }}-\mathrm{V}_{\text {min }}}{\mathrm{V}_{\text {nom }}}\right] \times 100
$$

Donde,

$\% \Delta V$ : máxima caída de tensión en el sistema.

$V_{\text {nom }}: \quad$ tensión fase-neutro nominal del sistema.

$V_{\min }: \quad$ mínima tensión presente en el sistema. 


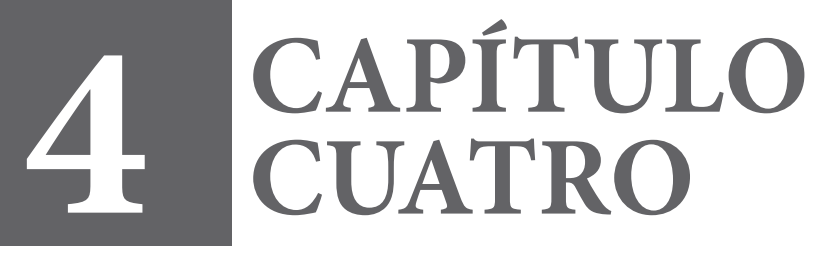




\section{Metodología para el Cálculo} DE PÉRDIDAS TÉCNICAS DE REDES EN MALLA A PARTIR DE FLUJO DE CARGA DE BARRIDO ITERATIVO

\subsection{Fundamentos de la metodología}

Con el fin de cálcular las pérdidas técnicas de potencia activa, se propone una metodología de flujo de carga para redes en malla que mantiene la topología de la red original (FIgurA NRO. 16) sin recurrir a equivalentes radiales. La metodología desarrollada considera un modelo trifásico del sistema e incorpora los mismos aspectos operativos contemplados en las redes radiales, tales como desbalance en la carga, faseo de los ramos de red, tipo de medidor, entre otros.

En la Figura NRo. 16 se muestra una red en malla típica, cuyos tramos y cargas tienen diferentes configuraciones de fases. Así, para el modelamiento, se considera una red trifásica tetrafilar o monofásica trifilar según sea el caso, es decir, no se consideran equivalentes monofásicos.

Figura NRO. 16. Modelo trifásico de red secundaria en malla.

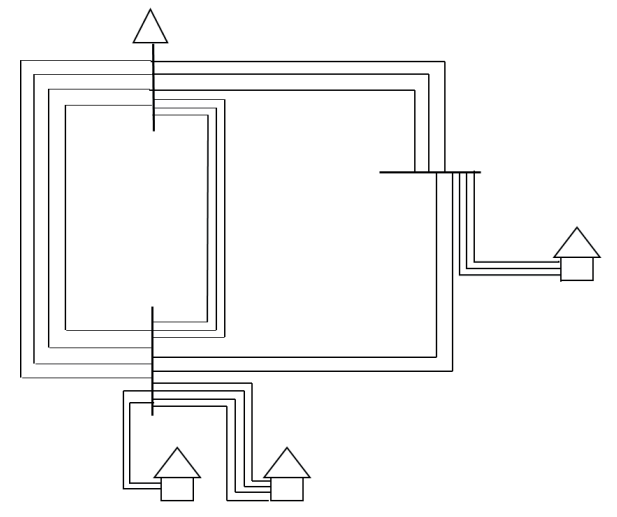


Entre las dificultades técnicas que se han encontrado para utilizar técnicas clásicas de flujo de carga - como el flujo de carga propuesto por Newton Raphson-, se destaca el hecho de que, debido a la alta radialidad de los circuitos, la matriz jacobiana presente dos situaciones:

i) Matrices muy grandes con pocos elementos diferentes de cero (matrices muy dispersas).

ii) Una diagonal débil debido a que tengan lugar pequeñas aberturas angulares entre nodos en algunos sistemas de distribución.

\subsection{Flujo de carga}

La base fundamental del método es un análisis circuital utilizando la segunda ley de Kirchhoff. Estanos lleva a la generalizada y bien conocida ley de Ohm en términos de tensiones y corrientes nodales. Aquella se expresa así:

$$
\left[I_{b u s}\right]=\left[Y_{b u s}\right]\left[V_{b u s}\right]
$$

La metodología consiste básicamente de tres pasos:

i) Aplicar segunda ley de Kirchhoff para encontrar las tensiones nodales.

ii) Calcular los flujos de corriente en todos los tramos.

iii) Calcular el error de corrección para corregir las cargas.

Estos tres pasos se aplican iterativamente hasta que el error sea minimizado, razón por la cual de aquí en adelante esta metodología es llamada «de aproximaciones sucesivas». 
Fundamentalmente, el método parte del análisis de un sistema desequilibrado y toma en cuenta los siguientes aspectos:

a. Se trabaja con la impedancia en forma matricial de cada tramo, considerando el material, calibre y montaje.

b. Se conforma la matriz $Y_{b u s}$ convencional del sistema, considerando todos los nodos y todas las fases.

$$
Y_{b u s}=\left[\begin{array}{ll}
{\left[Y_{a}\right]} & {\left[Y_{b}\right]} \\
{\left[Y_{c}\right]} & {\left[Y_{d}\right]}
\end{array}\right]
$$

Para el caso de circuitos alimentados por transformadores trifásicos, la matriz $Y_{b u s}$ es una matriz cuadrada de $(3 \times$ número de nodos $) \times(3 \times$ número de nodos $)$. Para circuitos alimentados por transformadores monofásicos, la matriz $Y_{\text {bus }}$ es una matriz cuadrada de $(2 \times$ número de nodos $) \times(2$ $\times$ número de nodos).

Donde,

$$
\begin{aligned}
Y_{a}=\left[Y_{1,1}\right]: \quad & \text { submatriz de tres filas por } \\
& \text { tres columnas, o dos filas } \\
& \text { por dos columnas según el } \\
& \text { transformador. } \\
& \\
& \text { submatriz de tres filas por } \\
& \text { tres (número de nodos }-1) \\
& \text { columnas o dos filas por } \\
& \text { dos (número de nodos }-1) \\
& \text { columnas según el tipo de } \\
& \text { transformador. }
\end{aligned}
$$




$$
\begin{gathered}
Y_{c}=\left[Y_{2,1}, Y_{3,1}, \ldots, Y_{n, 1}\right]^{T}: \begin{array}{l}
\text { submatriz de tres (número } \\
\text { de nodos }-1 \text { ) filas por tres } \\
\text { columnas o dos (número } \\
\text { de nodos }-1 \text { ) filas por dos } \\
\text { columnas según el tipo de } \\
\text { transformador. }
\end{array} \\
Y_{d}=\left[\begin{array}{ccc}
Y_{2,2} & \cdots & Y_{2, n} \\
\vdots & \ddots & \vdots \\
Y_{n, 2} & \cdots & Y_{n, n}
\end{array}\right]: \begin{array}{l}
\text { submatriz de tres (número } \\
\text { de nodos }-1 \text { ) filas por tres } \\
\text { (número de nodos }-1) \\
\text { columnas o dos (número } \\
\text { de nodos }-1 \text { ) filas por dos } \\
\text { (número de nodos }-1 \text { ) }
\end{array} \\
\begin{array}{ll}
\text { columnas según el tipo de } \\
\text { transformador. }
\end{array}
\end{gathered}
$$

La ecuación básica en términos de submatrices es:

$$
\left[\begin{array}{c}
I_{1} \\
I_{n c}
\end{array}\right]=\left[\begin{array}{ll}
{\left[Y_{a}\right]} & {\left[Y_{b}\right]} \\
{\left[Y_{c}\right]} & {\left[Y_{d}\right.}
\end{array}\right]\left[\begin{array}{c}
V_{1} \\
V_{n c}
\end{array}\right]
$$

Donde,

Subíndice 1: se refiere a la oscilante o barra del transformador.

nc: $\quad$ se refiere a los nodos de carga exceptuando el nodo del transformador.

c. Para cada nodo del circuito, se asigna un valor de carga en forma proporcional a la facturación; en forma similar a como se hace en el flujo radial, explicado en el capítulo anterior.

d. Inicialmente, se asumen todas las tensiones nodales en sus valores nominales. 
e. Con las cargas y las tensiones nodales, se obtiene las corrientes en los nodos de carga $I_{n c}$.

f. Se resuelve el sistema aplicando la ecuación (4.3) para encontrar las diferentes tensiones nodales de los nodos de carga.

$$
V_{n c}=\left(Y_{d}\right)^{-1}\left(I_{n c}-Y_{c} * V_{1}\right)
$$

g. Conocidas las tensiones nodales, se evalúa las corrientes del transformador de todas las fases, o $I_{l}$.

$$
I_{1}=Y_{a} V_{1}+Y_{b} V_{n c}
$$

h. Teniendo las corrientes del transformador, se calcula la potencia entregada por este, el cual se compara con un valor de referencia y este error se utiliza para modificar las cargas, e iniciar de nuevo el proceso (calcular las corrientes, las tensiones nodales y la corriente en el transformador). El proceso se repite hasta que el error sea menor a un valor preespecificado.

Para cada hora de un día típico (ordinario y festivo), se realiza un flujo de carga con el fin de obtener las pérdidas de potencia activa y verificar las condiciones operativas de cargabilidad de red y regulación de tensión. Con las 24 horas evaluadas, se obtienen las pérdidas técnicas de energía para el día típico ordinario y el día típico festivo. Las pérdidas de energía para el día ordinario y el día festivo se multiplican por la cantidad de días ordinarios y festivos que tenga el año. Esto dará como resultado las pérdidas de energía anual.

Dichos resultados de las pérdidas de energía anual se presentarán en relación con el circuito completo y sus componentes: transformador, red, acometidas y contadores. 


\subsection{Procedimiento}

Figura NRo. 17. Diagrama del flujo de carga para redes en malla.

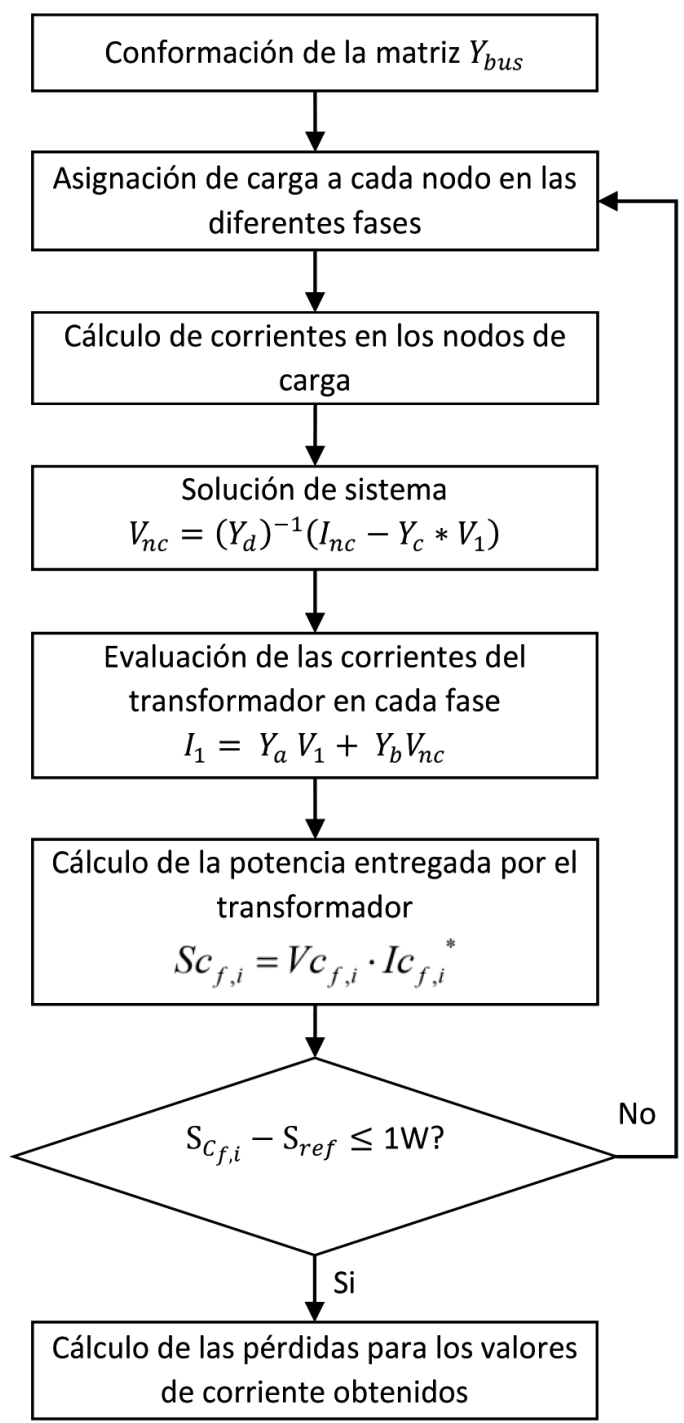

Una vez el proceso ha convergido, se calculan las pérdidas técnicas en los tramos de red secundaria con los datos de corrientes por fase del sistema; y las pérdidas en el transformador, los medidores y las acometidas. 


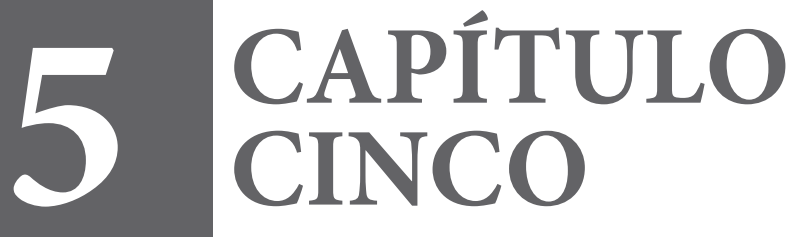




\section{Herramientas computacionales desarrolladas}

Las herramientas computacionales desarrolladas tienen procedimientos de revisión de datos y procedimientos de cálculo. Estas herramientas están constituidas por varios módulos funcionales que realizan subprocesos. Dichos módulos se elaboraron en Delphi ${ }^{\mathrm{Tx}}$ y Visual Studio ${ }^{\mathrm{Tm}}$. Los datos de entrada corresponden a la información constructiva y operativa de las redes secundarias (nivel de tensión 1) y sus usuarios. Estos deben estar descritos en archivos de Microsoft Excel $^{\oplus}$, con extensión .csv.

Estas herramientas se consolidan en dos tipos de software:

DETERED UTP: este aplicativo analiza la información constructiva de las redes secundarias para identificar inconsistencias que deben ser revisadas por el operador de red (OR) y así ejecutar flujos de carga solo en circuitos con información coherente.

PESIDIBT UTP: este aplicativo permite realizar los flujos de carga en circuitos radiales y en malla con información coherente. De este modo, muestra los resultados de pérdidas técnicas por circuito, totales o por componentes (red, transformadores, acometidas y medidores), y la información operativa útil para los análisis posteriores (cargabilidad del circuito, nodo con peor regulación de tensión).

La sección 5.1 muestra el diagrama de flujo general con el que se calculan las pérdidas técnicas en redes secundarias y las secciones posteriores muestran el detalle de cada uno de los subprocesos. 


\subsection{Diagrama de proceso}

En la FiguRA NRO. 18 se presenta el diagrama de proceso para la ejecución del cálculo de pérdidas en redes de nivel de tensión 1.

FigURA NRO. 18. Herramientas computacionales para el cálculo de pérdidas en el nivel 1.

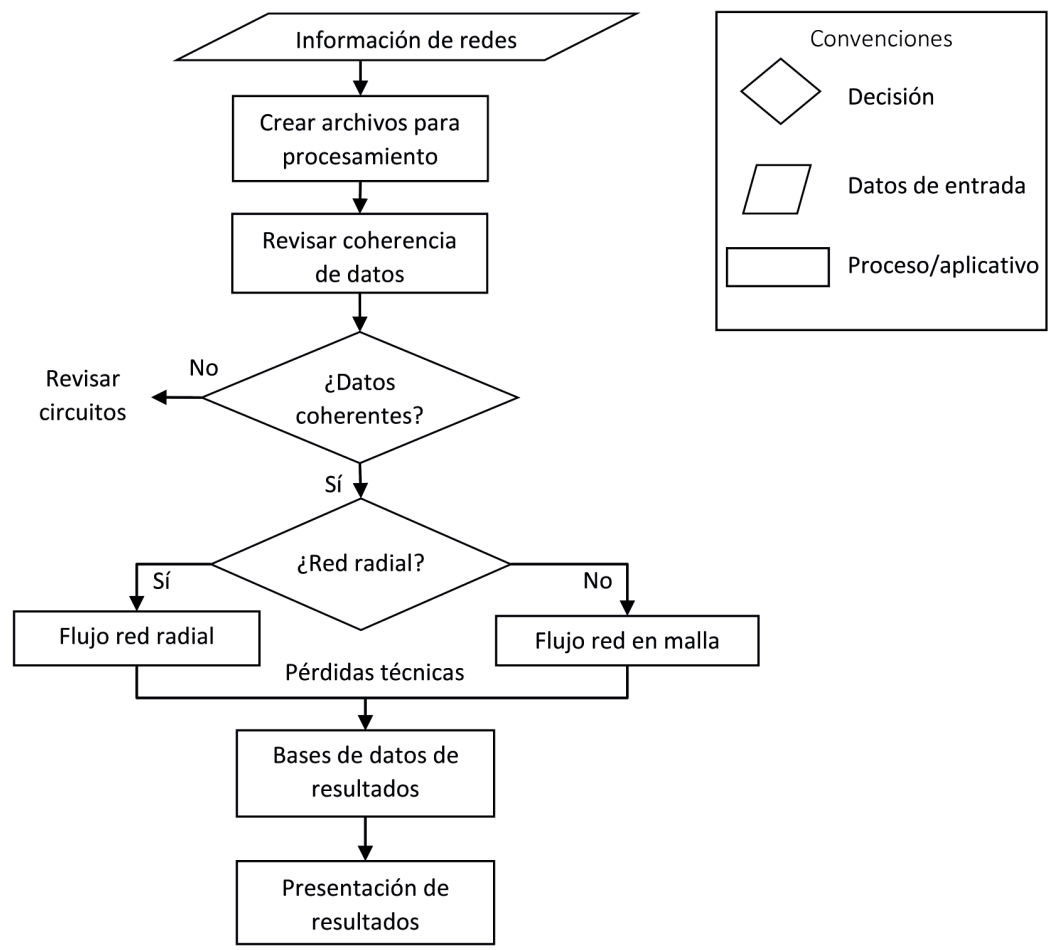

En la Figura NRO. 18 se presenta el diagrama de proceso para la ejecución del cálculo de pérdidas en redes de distribución secundaria. Los datos de entrada corresponden a la información constructiva y operativa de las redes secundarias y sus usuarios. Estos deben estar descritos en archivos de Microsoft Excel ${ }^{\circledR}$, en el formato que se describe en la sección 5.2. 
Un aplicativo es un software o código de computador que realiza transformación de los datos, procedimientos de revisión de los datos o de cálculo. Cada aplicativo está constituido por uno o más módulos funcionales que realizan subprocesos y se describirán en este capítulo conforme se vayan desarrollando. Estos aplicativos se elaboraron en $\mathrm{Microsoft}^{E^{2}}{ }^{\oplus}{ }^{\oplus}$, Delphi $^{\mathrm{TM}}$ y Visual Studio $^{\mathrm{Tx}}$.

Para redes secundarias, el proceso entrega como salida:

- El cálculo de pérdidas técnicas, total y por componentes de la red secundaria.

- Las condiciones operativas de la red (regulación, cargabilidad y desbalance).

\subsection{Estructura de los datos de entrada}

Aquello que se presentará a continuación aplica para las redes secundarias radiales o en malla que contienen información completa. A estas se les aplicará el procedimiento de cálculo de pérdidas técnicas mediante el flujo de carga.

La información se ingresa como cinco archivos de Microsoft Excel $^{\circledast}$ en formato delimitado por comas (.csv). Estos corresponden a curvas de carga de día típico ordinario, curvas de carga de día típico festivo, información de transformadores, tramos de red y usuarios conectados. Se utilizará el código o identificador del transformador como llave para asociar la información de curvas, usuarios y tramos a un circuito secundario. El contenido de cada archivo se explica en la TABLA NRO. 14. 
TABLA NRO. 14. Archivo de datos para redes. ${ }^{1}$

\begin{tabular}{|c|c|}
\hline \multicolumn{2}{|c|}{ Curvas_Ord.csvy Curvas_Fest.csv } \\
\hline Código del transformador & Código del transformador al cual se asociará la curva. \\
\hline$P$ trifásico horario (Hora1_P) & Potencia activa trifásica en la hora 1. \\
\hline $\bar{\vdots}$ & $\bar{\vdots}$ \\
\hline$P$ trifásico horario (Hora24_P) & Potencia activa trifásica en la hora 24. \\
\hline$f p$ horario (Hora1_fp) & Factor de potencia en la hora 1. \\
\hline$\vdots$ & $\vdots$ \\
\hline$f p$ horario (Hora24_fp) & Factor de potencia en la hora 24. \\
\hline \multicolumn{2}{|r|}{ Transformadores.csv } \\
\hline Código del transformador & \multirow{2}{*}{$\begin{array}{l}\text { Código que identifica el transformador. } \\
\text { Nodo donde está ubicado el transformador. Este nodo debe también estar } \\
\text { en la hoja tramos. }\end{array}$} \\
\hline Nodo & \\
\hline kVA nominales & Potencia nominal del transformador. \\
\hline Tipo (Monofásico / Trifásico) & Código: Monofásico (1) Trifásico (3). \\
\hline V. Primario & Tensión línea-línea en el primario [V]. \\
\hline V. Secundario & Tensión línea-línea en el secundario [V]. \\
\hline Topología red & ¿Es radial? / Código: \\
\hline Zona operativa* & Zona donde está ubicado / Código: 1 a 6 \\
\hline ID. municipio* & Código que identifica el municipio donde está ubicado el transformador. \\
\hline Urbano/Rural & 1 (Urbano) 2 (Rural). \\
\hline Coordenada $X^{*}$ & Coordenada $X$ de ubicación. \\
\hline Coordenada $Y^{*}$ & Coordenada $Y$ de ubicación. \\
\hline Altitud & Distancia vertical respecto al nivel del mar u otra referencia. \\
\hline Macromedición anual & Macromedición para anual. \\
\hline Indicador de diferencia anual & $\%$ anual. \\
\hline Consumo A.P. anual & Consumo de alumbrado público anual \\
\hline \multicolumn{2}{|r|}{ Tramos.csv } \\
\hline Código del transformador & Código del transformador al cual se asociará cada tramo. \\
\hline Nodo inicial & Nodo inicial de cada tramo de red. \\
\hline Nodo final & Nodo final de cada tramo de red. \\
\hline Faseo & $\begin{array}{l}\text { Fases que van por el tramo. Se asume que siempre va neutro. } \\
\text { Código: } \mathrm{AN}(1) \mathrm{BN}(2) \mathrm{CN}(3) \text {. } \\
\text { ABN (4) BCN (5) CAN (6). } \\
\text { ABCN (7). }\end{array}$ \\
\hline Longitud & Longitud en metros de cada tramo. \\
\hline Montaje & $\begin{array}{l}\text { Disposición del tramo de red. } \\
\text { Código: Abierta (1). } \\
\text { Junto (2) (Múltiples, o juntos por ducto) }\end{array}$ \\
\hline Calibre Fase & $\begin{array}{l}\text { Calibre de las fases. } \\
\text { Código: } 4 \text { - } 2-1 / 0(10)-2 / 0(20) \text {. }\end{array}$ \\
\hline Material Fase & $\begin{array}{l}\text { Material de las fases. } \\
\text { Código: Cobre (1) Aluminio (2). }\end{array}$ \\
\hline Calibre Neutro & $\begin{array}{l}\text { Calibre del neutro. } \\
\text { Código: } 4-2-1 / 0(10)-2 / 0(20) \text {. }\end{array}$ \\
\hline Material Neutro & $\begin{array}{l}\text { Material del neutro. } \\
\text { Código: Cobre (1) Aluminio (2). }\end{array}$ \\
\hline \multicolumn{2}{|r|}{ Usuarios.csv } \\
\hline Código del transformador & Código del transformador al cual se asociará cada usuario. \\
\hline Matricula & Identificador del usuario. \\
\hline Nodo & Nodo donde está ubicado el usuario. Debe existir en la hoja tramos. \\
\hline Calibre de acometida & Calibre de la acometida. \\
\hline Longitud de acometida & ngitud en metros de la acometida. \\
\hline
\end{tabular}

1 El asterisco $\left(^{*}\right)$ en los ítems de la primera columna hace referencia a la información que no es estrictamente necesaria para realizar los flujos de carga y calcular las pérdidas técnicas en el sistema de distribución secundaria; sin embargo, es información útil para realizar análisis del sistema cuando se conozcan sus pérdidas. 


\begin{tabular}{|c|c|}
\hline Fases de conexión & $\begin{array}{l}\text { Fases a la que está conectado el usuario. } \\
\text { Código: } \mathrm{AN}(1) \mathrm{BN}(2) \mathrm{CN}(3) \\
\text { ABN (4) BCN (5) CAN (6). } \\
\text { ABCN (7). }\end{array}$ \\
\hline Tipo de medidor & $\begin{array}{l}\text { Tipo de medidor. } \\
\text { Código: Electrónico (1) Mecánico (2). }\end{array}$ \\
\hline Estrato* & Estrato socioeconómico (1-6) - Si no es residencial (0). \\
\hline Clase de servicio* & $\begin{array}{l}\text { Clase de servicio. } \\
\text { Código: Residencial (1). } \\
\text { Comercial (2). } \\
\text { Industrial (3). } \\
\text { Oficial (4). }\end{array}$ \\
\hline Coordenada $X^{*}$ & Coordenada $X$ de ubicación. \\
\hline Coordenada $Y^{*}$ & Coordenada $Y$ de ubicación. \\
\hline Consumo anual & Consumo anual de energía. \\
\hline Cantidad de usuarios & Especifica la cantidad de usuarios. \\
\hline
\end{tabular}

Las figuras nro. 19, 20 y 21 muestran el entorno gráfico del software PESIDIBT UTP, en el cual se carga la información de circuitos en nivel de tensión 1 . Inicialmente, se abre un cuadro diálogo con el cual se accede a la carpeta donde están los archivos. Luego, el aplicativo realiza el proceso de carga de la información. Finalmente, el aplicativo muestra la información cargada para iniciar el proceso de revisión de la información y el respectivo cálculo de las pérdidas técnicas.

FIGURA NRO. 19. Botón y entorno gráfico para cargar la información en el software PESIDIBT UTP.

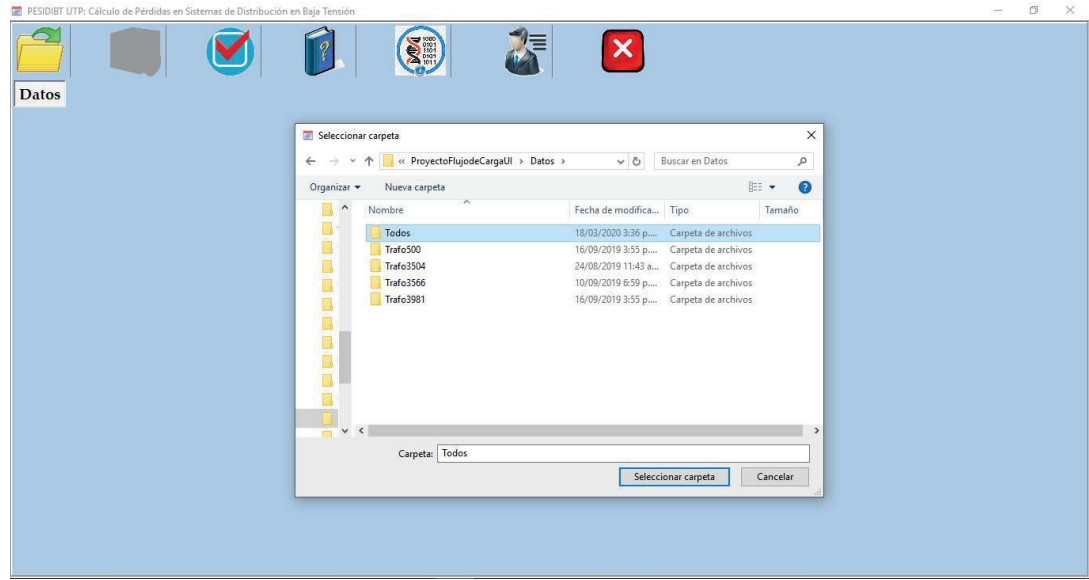


FIGURA NRO. 20. Entorno gráfico para cargar la información en el software PESIDIBT UTP.

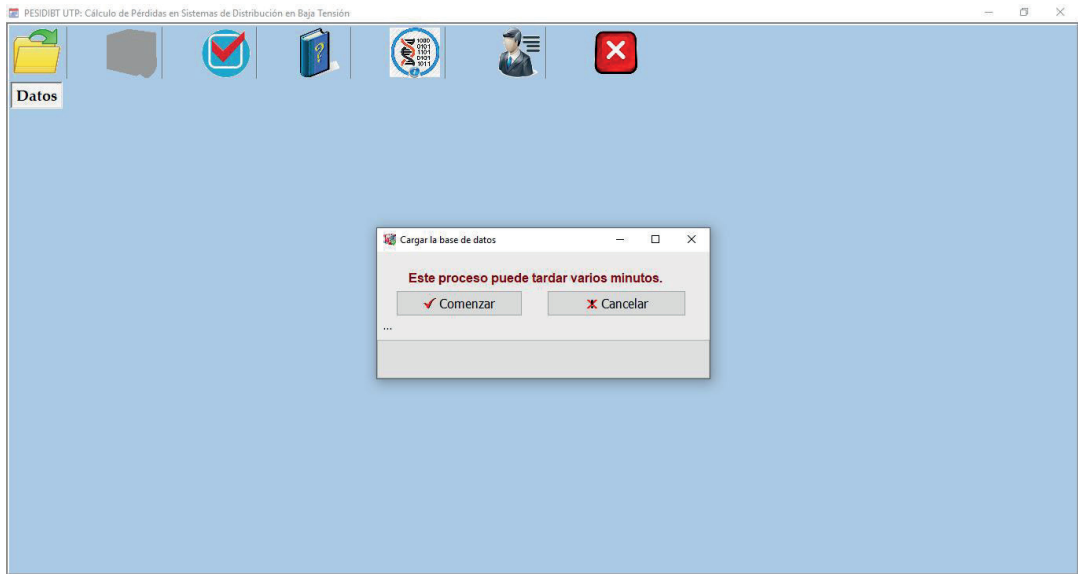

FIGURA NRO. 21. Entorno gráfico en que se muestra la información cargada en el software PESIDIBT UTP.

\begin{tabular}{|c|c|c|c|c|c|c|c|c|c|c|}
\hline 8 & 01 & & & & & & & & $-\quad 0$ & $x$ \\
\hline \multicolumn{11}{|l|}{ Datos } \\
\hline A Tramos & $\vartheta^{0}$ Ustatios & Trafos & $\rho^{9}$ curvas & & & & & & & \\
\hline Cod_Trafol & Nodo_Iniciall 1 & Nodo_Finall & Faseo & Longitud & Montaje & CalibreF ase & MaterialFase & CalibreNeutitc MaterialNeut & & A \\
\hline 14069 & 263961 & 263962 & 4 & 106.8 & 1 & 4 & 2 & 4 & & \\
\hline 14069 & 263962 & 288702 & 4 & 36.4 & 1 & 4 & 2 & 4 & & \\
\hline 14069 & 263963 & 288702 & 4 & 115.9 & 1 & 4 & 2 & 4 & & \\
\hline 14069 & 263964 & 263965 & 4 & 105.1 & 1 & 4 & 2 & 4 & & \\
\hline 14069 & 263965 & 263966 & 4 & 73.2 & 1 & 4 & 2 & 4 & & \\
\hline 14069 & 263966 & 263967 & 4 & 68.1 & 1 & 4 & 2 & 4 & & \\
\hline 14069 & 263967 & 263968 & 4 & 71.7 & 1 & 4 & 2 & 4 & & \\
\hline 14069 & 263968 & 264053 & 4 & 62.6 & 1 & 4 & 2 & 4 & & \\
\hline 14069 & 263967 & 263969 & 4 & 130.7 & 1 & 4 & 2 & 4 & & \\
\hline 14069 & 263969 & 299740 & 4 & 72.3 & 1 & 4 & 2 & 4 & & \\
\hline 14069 & 263967 & 263970 & 4 & 104.5 & 1 & 4 & 2 & 4 & & \\
\hline 14069 & 263970 & 275398 & 4 & 92.5 & 1 & 4 & 2 & 4 & & \\
\hline 14069 & 263970 & 288406 & 4 & 31 & 1 & 4 & 2 & 4 & & \\
\hline 14069 & 263970 & 263971 & 4 & 72.2 & 1 & 4 & 2 & 4 & & \\
\hline 14069 & 263971 & 288405 & 4 & 104.5 & 1 & 4 & 2 & 4 & & \\
\hline 14069 & 263971 & 288407 & 4 & 155.5 & 1 & 4 & 2 & 4 & & \\
\hline 14069 & 263971 & 263972 & 4 & 166 & 1 & 4 & 2 & 4 & & \\
\hline 14069 & 263972 & 293641 & 4 & 86.9 & 1 & 4 & 2 & 4 & & \\
\hline 14069 & 263971 & 275542 & 4 & 108.6 & 1 & 4 & 2 & 4 & & \\
\hline 14163 & 263995 & 263996 & 1 & 956 & 1 & 4 & 2 & 4 & & $\checkmark$ \\
\hline
\end{tabular}




\subsection{Procedimiento para revisar coherencia de los datos}

FiguRA NRO. 22. Diagrama de flujo del proceso para revisar coherencia de los datos.

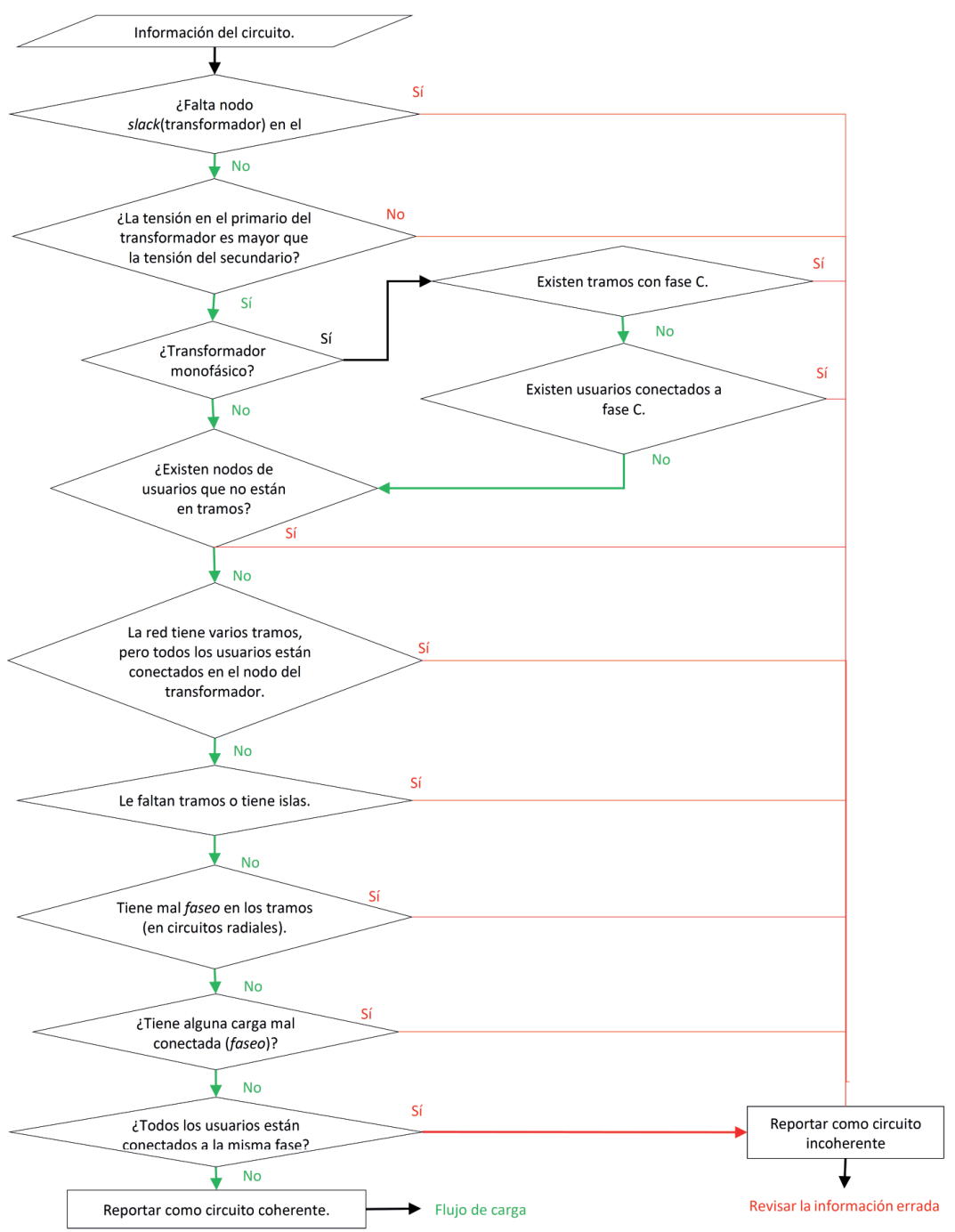

En la Figura NRO. 22 se presenta el diagrama de flujo del procedimiento del proceso para revisar la coherencia de la información constructiva y operativa de los circuitos secundarios. 
En este procedimiento, se realizan verificaciones tales como coherencia en faseo de tramos, usuarios y tipo de transformador; conectividad de cargas en cada circuito - es decir, que no falten tramos y por esto se generen islas-; y coherencia en nodos a fin de obtener dos listas de circuitos. A saber:

Circuitos con inconsistencias encontradas: estos circuitos deben ser revisados por el OR para corregir la información inconsistente.

Circuitos coherentes: estos circuitos quedan listos para la ejecución en el flujo de carga y con ellos calcular sus pérdidas.

En la Tabla NRO. 15 se listan las inconsistencias encontradas por el programa y su número de identificación.

TABLA NRO. 15. Lista de inconsistencias.

\begin{tabular}{|c|l|}
\hline $\begin{array}{c}\text { Código de } \\
\text { identificación }\end{array}$ & \\
\hline 0 & No hay información de tramos y tampoco usuarios. \\
\hline 1 & No hay información de usuarios. \\
\hline 2 & No hay información de curvas días ordinarios. \\
\hline 3 & No hay información de curvas días festivos. \\
\hline 4 & La curva de carga del día ordinario está en cero todas las horas. \\
\hline 5 & La curva de factor de potencia del día ordinario está en cero todas las horas. \\
\hline 6 & La curva de carga del día festivo está en cero todas las horas. \\
\hline 7 & La curva de factor de potencia del día festivo está en cero todas las horas. \\
\hline 8 & El nodo slack (transformador) no aparece en tramos. \\
\hline 9 & No hay tramos, pero si usuarios en el nodo slack. \\
\hline 10 & Existen nodos de usuarios que no están en tramos. \\
\hline 11 & Tiene tramos y todos los usuarios están en el transformador. \\
\hline 12 & Consumo total de usuarios del circuito en cero. \\
\hline 13 & Existen tramos cuyo nodo inicial y nodo final es el mismo. \\
\hline 14 & El valor nominal del transformador no coincide con el tipo. \\
\hline 15 & Existen códigos desconocidos en el tipo de transformador. \\
\hline 16 & El voltaje del primario del transformador es menor al voltaje secundario del \\
transformador. \\
\hline 17 & Existen códigos desconocidos en la topología del transformador. \\
\hline 18 & Existen códigos desconocidos en faseo de tramos. \\
\hline 19 & Existen códigos desconocidos en el montaje de tramos. \\
\hline 20 & Existen códigos desconocidos en el material de fases. \\
\hline 21 & Existen códigos desconocidos en el material de neutro. \\
\hline 22 & Calibres desconocidos en las fases. \\
\hline & \\
\hline
\end{tabular}




\begin{tabular}{|l|l|}
\hline 23 & Calibres desconocidos en el neutro. \\
\hline 24 & Existen códigos desconocidos en el tipo de medidor. \\
\hline 25 & Existen códigos desconocidos en el estrato (1 a 6). \\
\hline 26 & Existen códigos desconocidos en la clase de servicio (0 a 4). \\
\hline 27 & Consumo del usuario en cero. \\
\hline 28 & Ramas desconectadas. \\
\hline 29 & Nodos desconectados. \\
\hline 30 & Es radial y viene marcado como en malla. \\
\hline 31 & Tiene anillos y no debería porque está marcado como radial. \\
\hline 32 & El tramo anterior no tiene las fases para el actual. \\
\hline 33 & Tramo de la red no coinciden con el tipo de transformador. \\
\hline 34 & La acometida del usuario no corresponde con la red existente. \\
\hline 35 & Todos los usuarios están conectados a una sola fase. \\
\hline
\end{tabular}

La Figura NRO. 23 muestra un ejemplo de la visualización del reporte en el aplicativo DETERED UTP. Allí, se puede observar cómo el aplicativo muestra el código del transformador (circuito) que presenta incoherencia en la información y el tipo de error que presenta, junto con una breve descripción del error.

FIGURA NRO. 23. Visualización de DETERED UTP para ubicar inconsistencias en la información de circuitos en nivel de tensión 1.

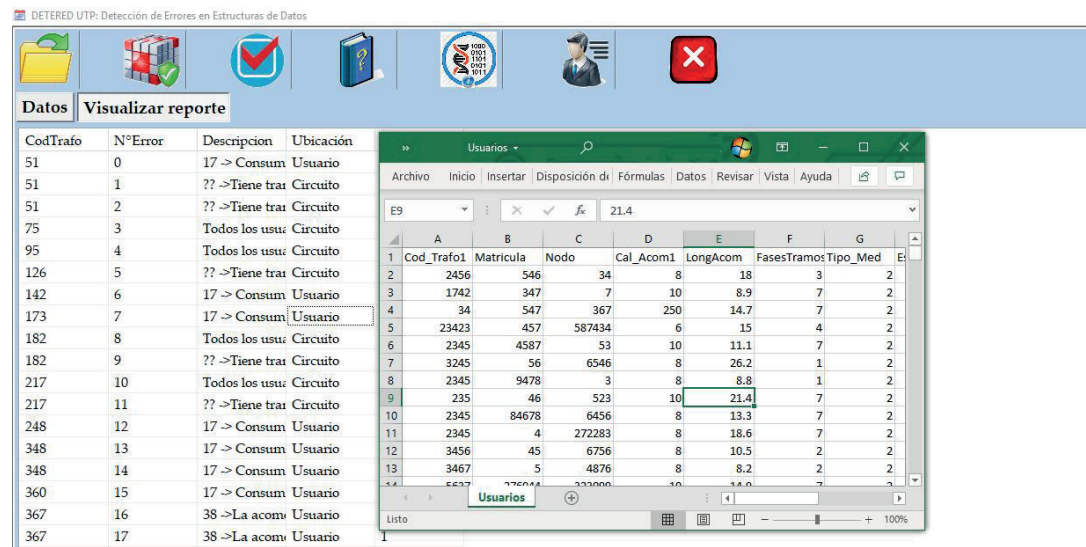

\subsection{Aplicativo para flujo de carga radial desbalanceado}

En la Figura NRO. 24, se presenta el diagrama de flujo del aplicativo de flujo de carga radial desbalanceado (modelamiento trifásico). 
Figura NRO. 24. Procedimiento del aplicativo para flujo de carga radial.

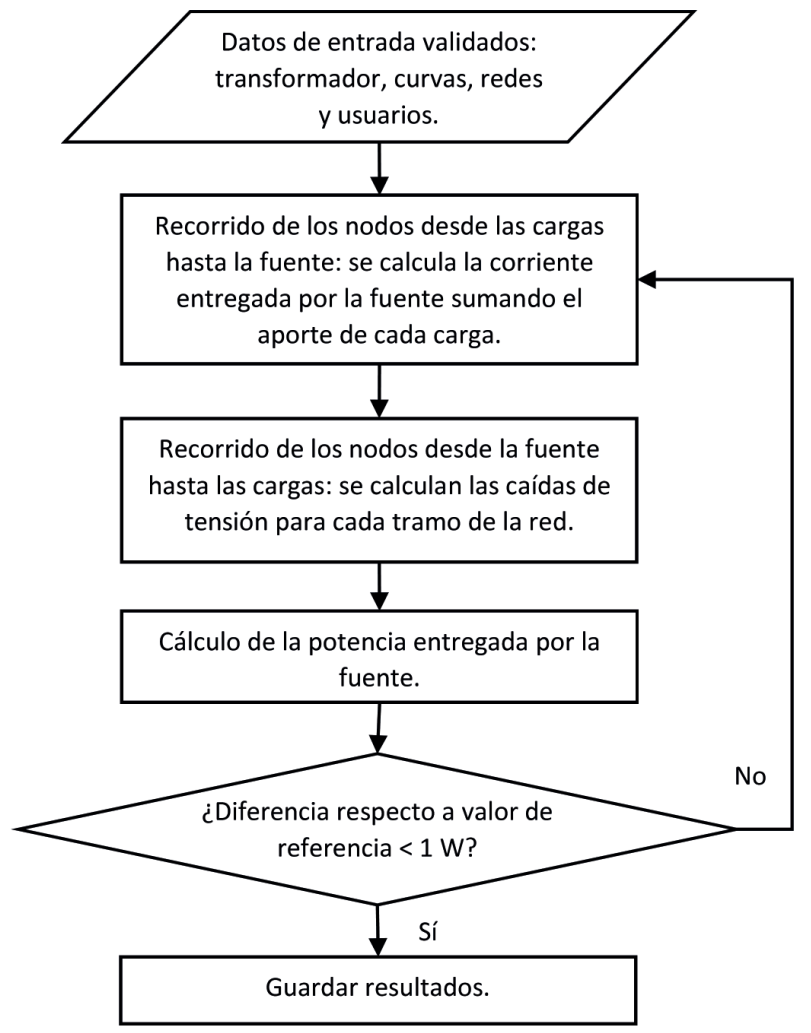

Este aplicativo está elaborado en Delphi ${ }^{\mathrm{rx}}$. Los datos de entrada (Curvas.csv, Tramos.csv, Trafos.csv y Usuarios.CSV) corresponden a los circuitos coherentes reportados en el proceso descrito en la Figura NRO. 22. Los resultados obtenidos de cada transformador quedan registrados en un archivo llamado CircuitosNormales. Csv. Allí, se consolidan los resultados de todos los transformadores.

\subsection{Aplicativo para flujo de carga de red en malla}

En la Figura NRo. 25 se presenta el diagrama de flujo del aplicativo para calcular pérdidas en circuitos con red en malla. Este aplicativo se desarrolla en Delphi ${ }^{\mathrm{Tm}} \mathrm{y}$ toma archivos de 
usuarios, transformadores, curvas, tramos y entrega resultados de pérdidas.

Figura NRO. 25. Procedimiento del aplicativo para flujo de red en malla.

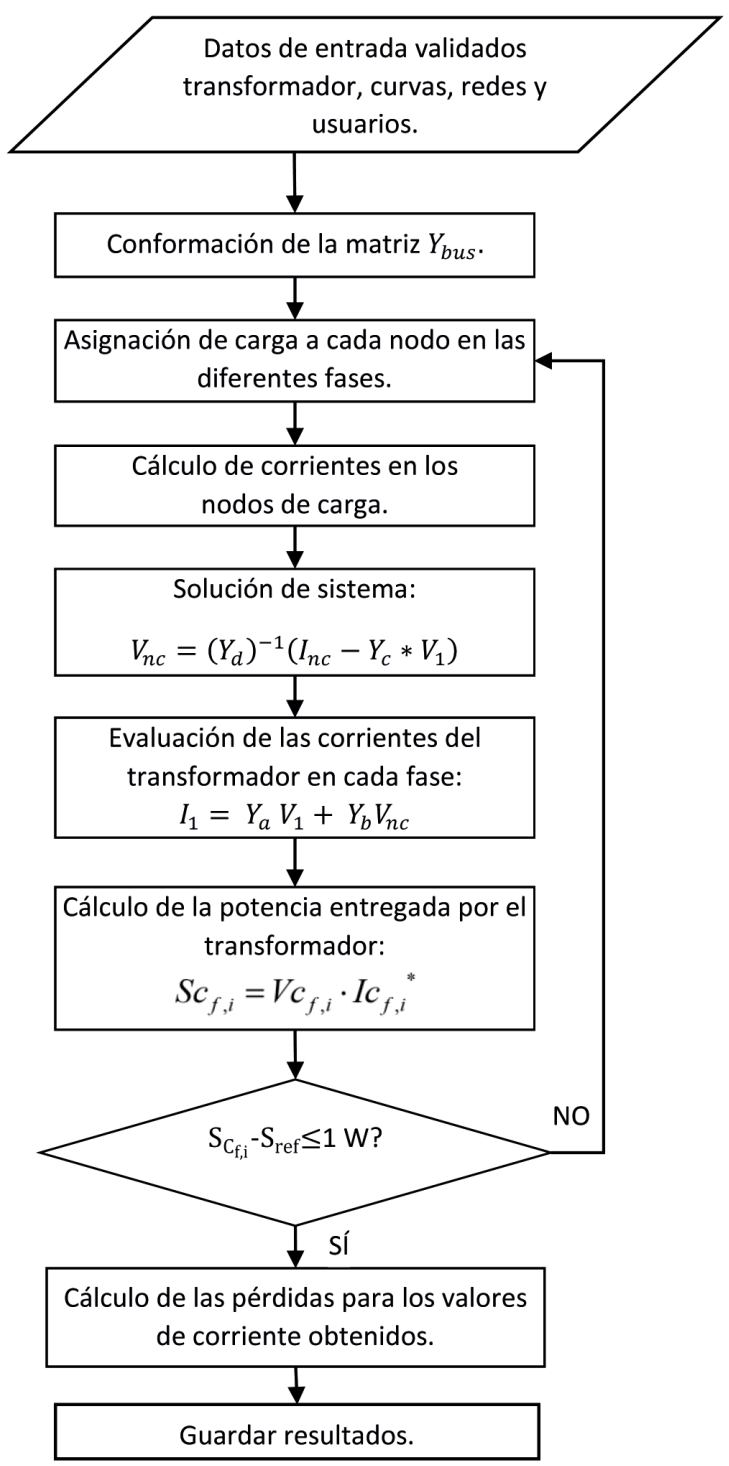




\subsection{Software y muestra de resultados}

El software desarrollado se llama PESIDIBT UTP, la FIGURA NRO. 26 muestra el botón con el que se realiza la ejecución del flujo de carga para el cálculo de las pérdidas técnicas. Asimismo, la FIgURA NRO. 27 muestra una ventana emergente en la cual se configura la cantidad de días festivos y ordinarios que se quieren utilizar en el cálculo de pérdidas técnicas anuales.

Figura NRo. 26. Botón para realizar la ejecución del cálculo de pérdidas técnicas.

PESIDIBT UTP: Cálculo de Pérdidas en Sistemas de Distribución en Baja Tensión

Figura NRo. 27. Ejecución del cálculo de pérdidas técnicas.

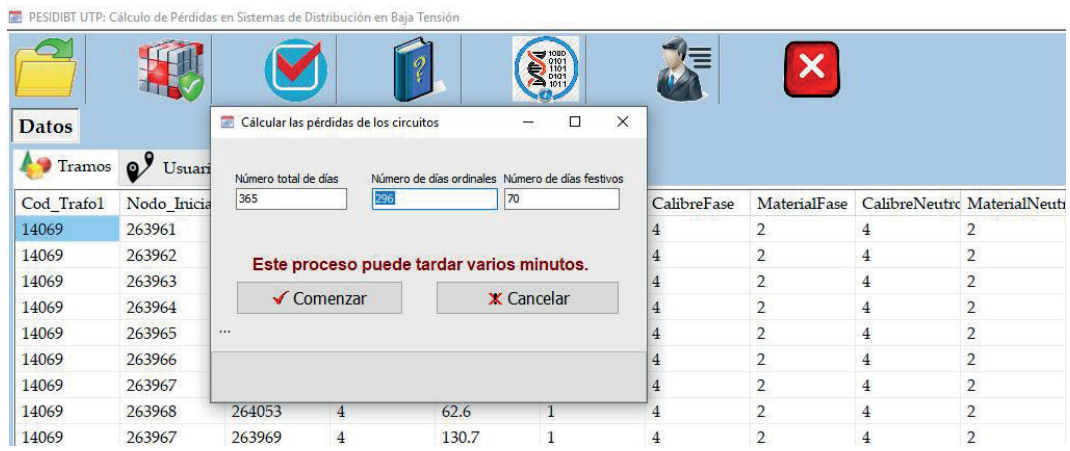

Los resultados del programa se almacenan en una base de datos, en Access ${ }^{\circledast}$, y se dispone de un aplicativo desarrollado en Visual Studio ${ }^{\text {Tx }}$ que permite consultar, para un circuito secundario, los resultados de pérdidas técnicas totales y por componentes. También, se pueden consultar las condiciones operativas resultantes del flujo de carga. El propósito de esta base de datos y de su programa de consulta consiste en poderse revisar con detalle la información de los circuitos secundarios (utilizados para realizar el estudio) y sus resultados. Adicionalmente, el interesado puede realizar consultas en Access ${ }^{\circledast}$ mediante instrucciones SQL. 
Este aplicativo se ejecuta desde el software PESIDIBT UTP. La Figura NRO. 28 muestra el botón de consulta del aplicativo y la ventana emergente donde se visualizan los resultados del programa. Allí, no solo se pueden consultar los resultados inmediatamente obtenidos tras correr el flujo de carga, sino que además se pueden observar los resultados guardados de secuencias anteriores.

FIGURA NRO. 28. Botón y ventana de los resultados de pérdidas técnicas en nivel de tensión 1.

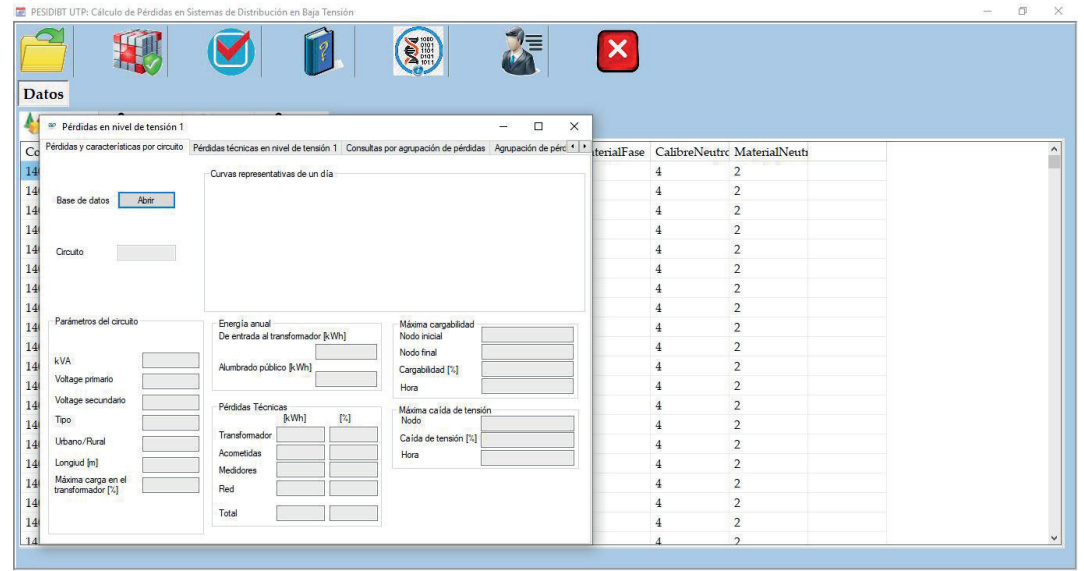

En la pestaña «Pérdidas y características por circuito» se puede consultar la información individual de cada circuito de nivel de tensión 1, tal como lo muestra la Figura NRO. 28.

Se muestran características del circuito como la capacidad nominal, el tipo de transformador, la localización (urbano/rural), la longitud del circuito, la máxima carga del transformador, la curva de carga para un día ordinario y festivo, la energía de entrada al circuito, las pérdidas técnicas, el tramo de red con máxima cargabilidad y el nodo con máxima caída de tensión. 
Figura NRO. 29. Visualización de los resultados de pérdidas técnicas N1 por circuito.

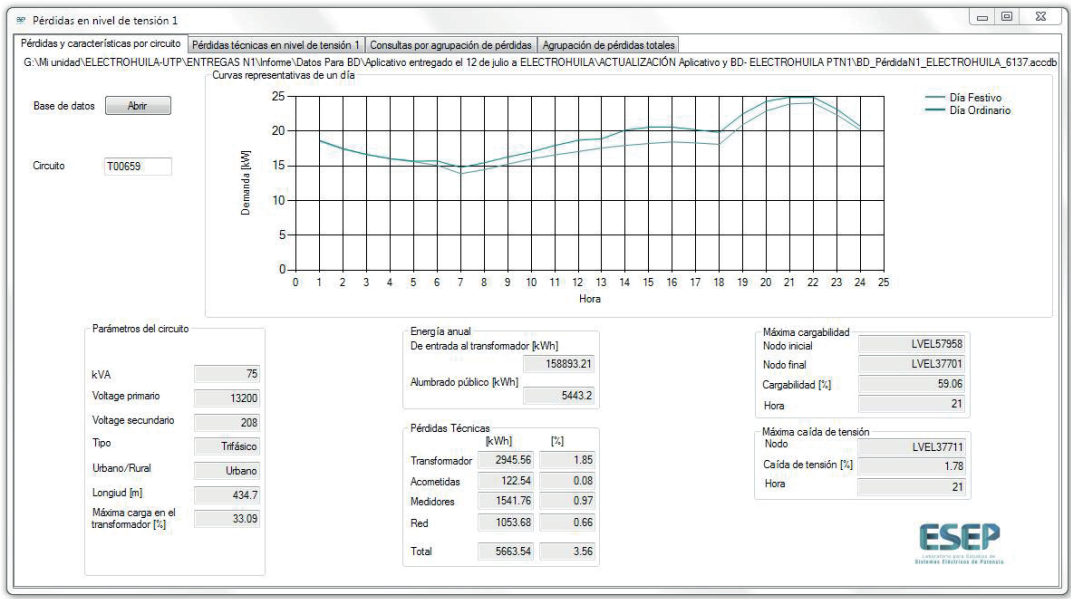

En la pestaña «Pérdidas técnicas en nivel de tensión 1», se pueden consultar los índices de pérdidas técnicas totales del estudio y por componente (ver FigURA NRO. 30).

FiguRA NRO. 30. Visualización de los resultados totales de pérdidas técnicas N1.

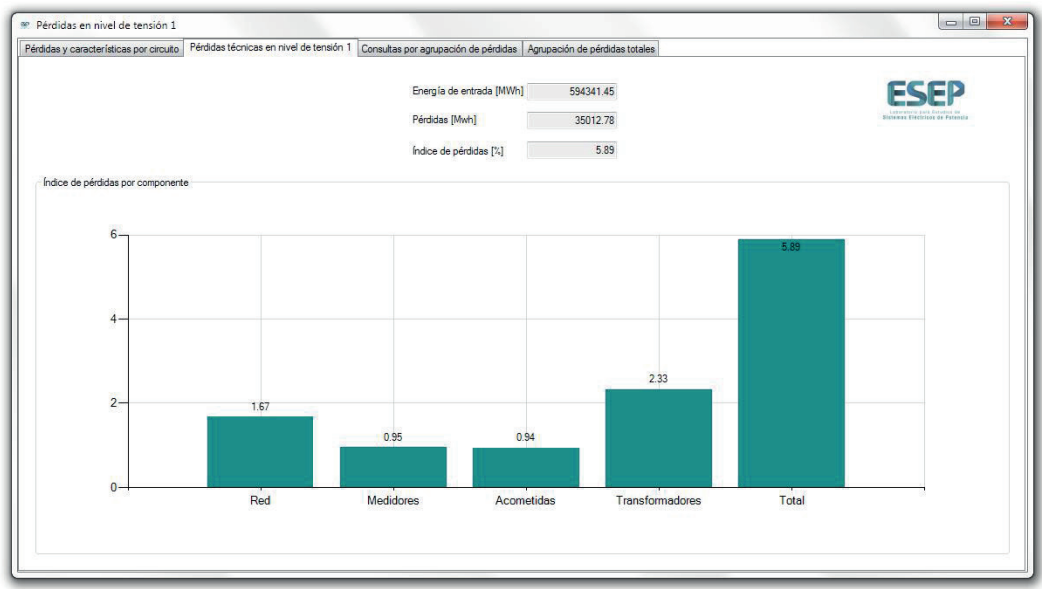


En la pestaña «Consulta por agrupación de pérdidas», se pueden realizar consultas para conocer las pérdidas técnicas de un conjunto de transformadores en particular. Así mismo, se pueden conocer los índices de pérdidas por componente según la combinación por tipo de transformador, localización, zona y municipio escogida por el usuario. Por ejemplo, se podría consultar las pérdidas de circuitos monofásicos, urbanos, de un municipio en concreto, tal como lo muestra la FIGURA NRO. 31.

FIGURA NRO. 31. Visualización de los resultados de pérdidas técnicas N1 por agrupación de localización, tipo de transformador, zona geográfica y municipio.

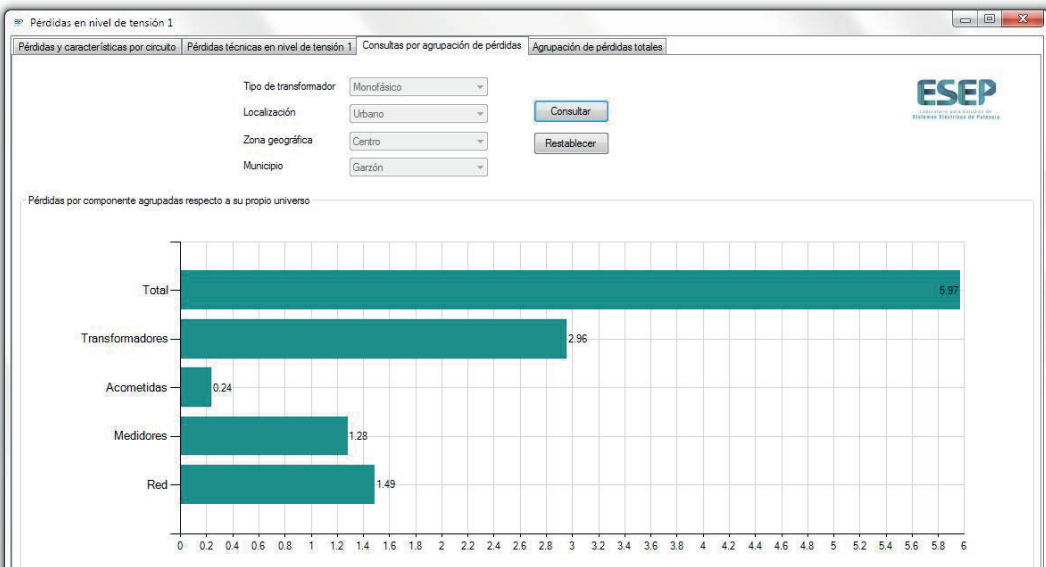

A través de la pestaña «Agrupación de pérdidas totales», se pueden comparar los índices de las pérdidas totales por localización, el tipo de transformador, la topología de red, la zona geográfica o el municipio, como se muestra en la FIGURA NRO. 32. 
FigURA NRO. 32. Visualización de los resultados de pérdidas técnicas N1 por agrupación de localización, tipo de transformador, topología de red, zona geográfica o municipio.

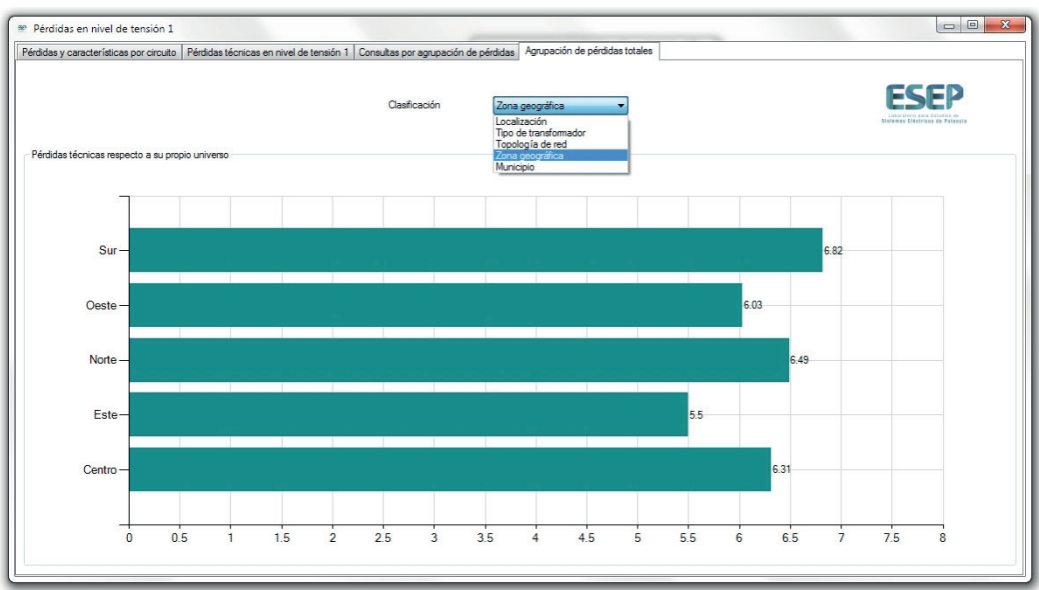




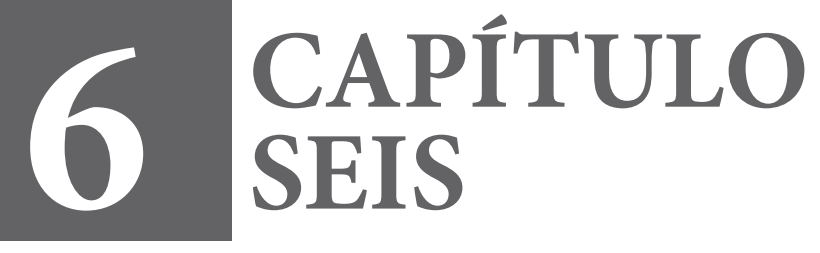




\section{Ejemplos y casos de prueba}

Con el fin de mostrar los resultados obtenidos con la metodología propuesta y el software desarrollado, se creó un sistema de distribución ficticio el cual tiene cuatro circuitos de distribución. A estos circuitos de prueba se les calculó las pérdidas técnicas con la herramienta computacional desarrollada (PESIDITB UTP).

Los circuitos elegidos tienen las siguientes características (TABLA NRO. 16) un circuito radial alimentado por un transformador trifásico, un circuito radial alimentado por un transformador monofásico, un circuito mallado alimentado por un transformador monofásico y un circuito mallado alimentado por un transformados trifásico. Se consideran distintos usos de fases en usuarios y en los tramos de red con el fin mostrar la versatilidad que tiene la metodología y el software desarrollado.

TABLA NRO. 16. Circuitos de prueba para cálculo de pérdidas técnicas.

\begin{tabular}{|c|l|l|c|c|c|c|c|}
\hline Circuito & $\begin{array}{c}\text { Tipo de } \\
\text { transformador }\end{array}$ & Topología & $\begin{array}{c}\text { Zona } \\
\text { operativa }\end{array}$ & $\begin{array}{c}\text { Longitud } \\
{[\mathbf{m}]}\end{array}$ & $\begin{array}{c}\text { Número } \\
\text { de } \\
\text { usuarios }\end{array}$ & $\begin{array}{c}\text { Tiene } \\
\text { AP }\end{array}$ & $\begin{array}{c}\text { Número de } \\
\text { tramos }\end{array}$ \\
\hline 1 & Trifásico & Radial & 2 & 414 & 22 & Sí & 23 \\
\hline 2 & Monofásico & Radial & 2 & 19.2 & 10 & No & 3 \\
\hline 3 & Monofásico & Mallado & 3 & 182.9 & 30 & No & 12 \\
\hline 4 & Trifásico & Mallado & 1 & 531.6 & 30 & Sí & 26 \\
\hline
\end{tabular}

Inicialmente, se presentan los resultados de pérdidas técnicas para cada uno delos circuitos propuestos. Posteriormente, se presentan los resultados a nivel de sistema, considerando que el sistema de distribución ficticio tiene cuatro transformadores de distribución, ubicados en tres zonas operativas ficticias. Así, se presentarán los resultados de las pérdidas técnicas del sistema de distribución, clasificadas de acuerdo con características comunes de los circuitos. 


\subsection{Circuito de prueba 1}

La Figura NRo. 33 muestra la topología del circuito simulado y la TABLA NRO. 17 presenta la información de los usuarios conectados al circuito de distribución 1.

Figura NRO. 33. Topología del circuito 1.

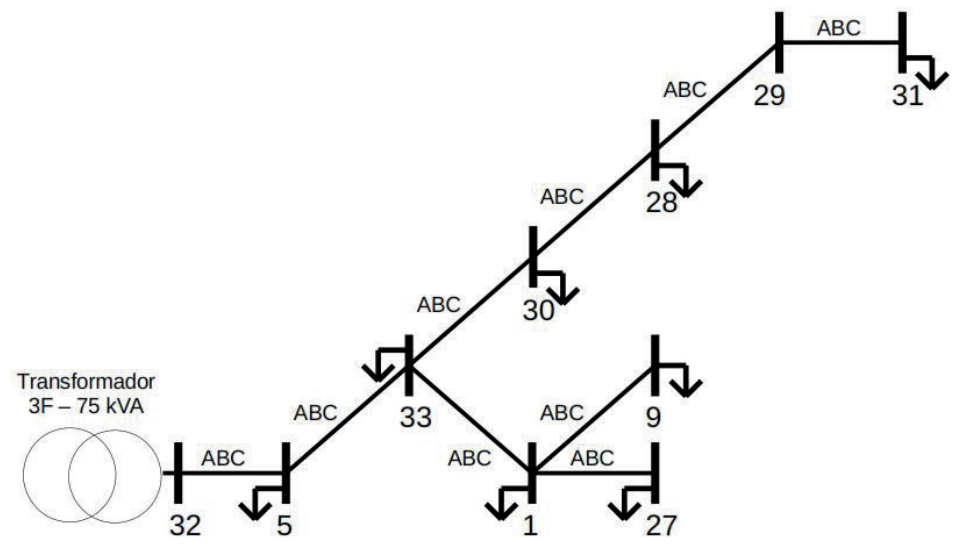

TABLA NRO. 17. Información de usuarios conectados al circuito 1.

\begin{tabular}{|c|c|c|c|c|c|}
\hline Nodo & $\begin{array}{c}\text { Calibre } \\
\text { Acometida }\end{array}$ & $\begin{array}{c}\text { Longitud } \\
\text { Acometida }[\mathbf{m}]\end{array}$ & Conexión & $\begin{array}{c}\text { Tipo de } \\
\text { Medidor }\end{array}$ & $\begin{array}{c}\text { Consumo } \\
\text { anual } \\
\mathbf{k W h}\end{array}$ \\
\hline 9 & 8 & 12.2 & $\mathrm{AN}$ & Mecánico & 4159.5 \\
\hline 9 & 8 & 8.7 & $\mathrm{BN}$ & Mecánico & 91.5 \\
\hline 30 & 8 & 12.6 & $\mathrm{CN}$ & Mecánico & 2016 \\
\hline 28 & 8 & 16.5 & $\mathrm{AN}$ & Mecánico & 3844.5 \\
\hline 31 & 8 & 17.9 & $\mathrm{AN}$ & Electrónico & 1996.5 \\
\hline 31 & 8 & 11.1 & $\mathrm{AN}$ & Mecánico & 1837.5 \\
\hline 31 & 8 & 16.3 & $\mathrm{AN}$ & Mecánico & 1530 \\
\hline 9 & 8 & 18.3 & $\mathrm{AN}$ & Mecánico & 4750.5 \\
\hline 5 & 8 & 16.1 & $\mathrm{AN}$ & Mecánico & 2287.5 \\
\hline 5 & 8 & 15.8 & $\mathrm{ABCN}$ & Mecánico & 6732 \\
\hline 28 & 8 & 11.9 & $\mathrm{BCN}$ & Mecánico & 5583 \\
\hline 30 & 8 & 8.7 & $\mathrm{ABCN}$ & Mecánico & 56155.5 \\
\hline 33 & 8 & 9.2 & $\mathrm{ABCN}$ & Mecánico & 12556.5 \\
\hline 31 & 8 & 14.1 & $\mathrm{ABCN}$ & Mecánico & 5070 \\
\hline 1 & 8 & 24 & $\mathrm{CN}$ & Mecánico & 871.5 \\
\hline 27 & 8 & 17.8 & $\mathrm{ABCN}$ & Mecánico & 5940 \\
\hline 30 & 8 & 9.7 & $\mathrm{CN}$ & Electrónico & 4687.5 \\
\hline 30 & 8 & 13.4 & $\mathrm{AN}$ & Mecánico & 1851 \\
\hline
\end{tabular}




\begin{tabular}{|c|c|c|c|c|c|}
\hline 28 & 8 & 4.1 & $\mathrm{ABCN}$ & Mecánico & 30285 \\
\hline 28 & 8 & 15.2 & $\mathrm{AN}$ & Mecánico & 4080 \\
\hline 1 & 8 & 11.1 & $\mathrm{AN}$ & Mecánico & 1336.5 \\
\hline 5 & 8 & 8 & $\mathrm{ABCN}$ & Electrónico & 13000.5 \\
\hline
\end{tabular}

La Figura NRO. 34 muestra algunos parámetros descriptivos del circuito de prueba 1 .

Figura NRO. 34. Parámetros descriptivos del circuito 1.

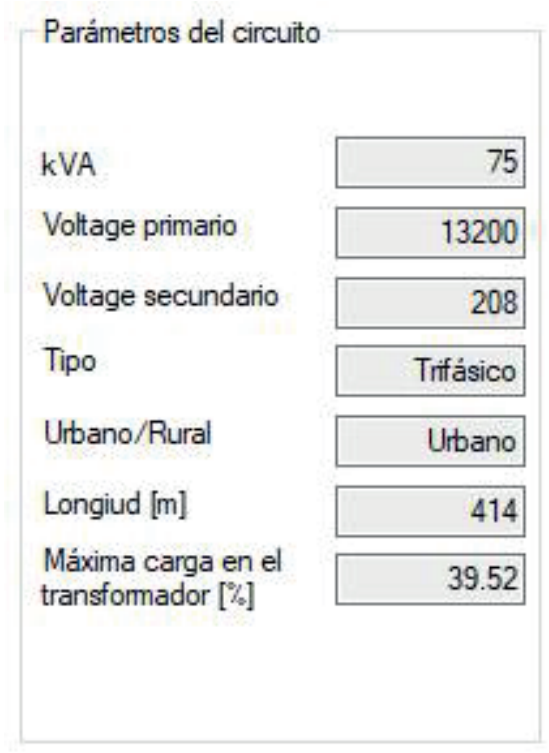

La FIgURA NRO. 34 muestra que el transformador presenta una cargabilidad del $39.52 \%$ en la hora de demanda máxima. Debido a que el transformador no tiene alta cargabilidad, este presentará un porcentaje de pérdidas representativo en el circuito.

En la Figura NRo. 35 se observa la energía anual de entrada al transformador, el consumo por alumbrado público y las pérdidas técnicas del circuito, desglosadas por componente (en valores reales y en porcentaje). 
FiguRA NRO. 35. Energía anual y pérdidas técnicas del circuito 1.

\begin{tabular}{|c|c|c|}
\hline \multicolumn{3}{|c|}{$\begin{array}{l}\text { Energia anual } \\
\text { De entrada al transformador }[k W h]\end{array}$} \\
\hline & & 195577.64 \\
\hline \multicolumn{3}{|c|}{ Alumbrado público [kWh] } \\
\hline Pérdidas Técni & & \\
\hline & [kWh] & [\%] \\
\hline Transformador & 3247.65 & 1.66 \\
\hline Acometidas & 439.77 & 0.22 \\
\hline Medidores & 468.66 & 0.24 \\
\hline Red & 4292.19 & 2.19 \\
\hline Total & 8448.27 & 4.32 \\
\hline
\end{tabular}

Como se puede observar, el elemento con mayor porcentaje de pérdidas técnicas es la red de distribución con un porcentaje del $2.19 \%$. En segundo lugar, se encuentra el transformador con un porcentaje del $1.66 \%$, y finalmente las acometidas y medidores con un porcentaje de pérdidas del $0.22 \%$ y $0.24 \%$, respectivamente.

En la Figura NRO. 36 se observa la máxima cargabilidad y la máxima caída de tensión para el circuito 1 .

FIGURA NRO. 36. Máxima cargabilidad y caída de tensión para el circuito 1.

\begin{tabular}{|c|c|}
\hline \multicolumn{2}{|l|}{ Máxima cargabilidad } \\
\hline Nodo inicial & 5 \\
\hline Nodo final & 33 \\
\hline Cargabilidad [\%] & 58.92 \\
\hline Hora & 21 \\
\hline \multicolumn{2}{|c|}{ Máxima caida de tensión } \\
\hline Nodo & 31 \\
\hline Caida de tensión [\%] & 5.22 \\
\hline Hora & 21 \\
\hline
\end{tabular}


Como se puede observar, la hora de mayor cargabilidad para el circuito es la 21. A esta hora, la cargabilidad máxima de la red ocurre entre los nodos 5 y 33 con un valor del $58.92 \%$. Este tramo de red es el más cargado ya que alimenta la mayor parte del circuito y tiene un calibre nro. 2, comparado con la salida del transformador (tramo entre los nodos 32 y 5 ), el cual se encuentra en calibre $1 / 0$. Por otra parte, la caída de tensión máxima es del $5.22 \%$ en el nodo 31 , el cual corresponde a una cola del circuito.

\subsection{Circuito de prueba 2}

La Figura NRO. 37 muestra la topología del circuito simulado y la TABLA NRO. 28 presenta la información de los usuarios conectados al circuito de distribución 2

FiguRA NRO. 37. Topología del circuito 2.

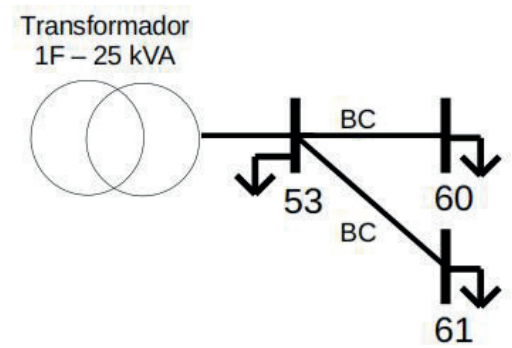

TABLA NRO. 18. Información de usuarios conectados al circuito 2.

\begin{tabular}{|c|c|c|c|c|c|}
\hline Nodo & $\begin{array}{c}\text { Calibre } \\
\text { Acometida }\end{array}$ & $\begin{array}{c}\text { Longitud } \\
\text { Acometida } \\
{[\mathbf{m}]}\end{array}$ & Conexión & $\begin{array}{c}\text { Tipo de } \\
\text { Medidor }\end{array}$ & $\begin{array}{c}\text { Consumo } \\
\text { anual } \\
\mathbf{k W h}\end{array}$ \\
\hline 60 & 8 & 6 & $\mathrm{BN}$ & Mecánico & 938 \\
\hline 61 & 8 & 8.3 & $\mathrm{BN}$ & Electrónico & 15400 \\
\hline 60 & 8 & 5 & $\mathrm{CN}$ & Electrónico & 29680 \\
\hline 60 & 8 & 7.8 & $\mathrm{CN}$ & Mecánico & 9884 \\
\hline 61 & 8 & 8 & $\mathrm{BN}$ & Mecánico & 4816 \\
\hline 61 & 8 & 6.1 & $\mathrm{BN}$ & Mecánico & 7098 \\
\hline 61 & 8 & 11.5 & $\mathrm{CN}$ & Mecánico & 6384 \\
\hline 53 & 8 & 8.5 & $\mathrm{CN}$ & Mecánico & 6244 \\
\hline 53 & 8 & 12 & $\mathrm{BN}$ & Mecánico & 5348 \\
\hline 61 & 8 & 1.5 & $\mathrm{CN}$ & Mecánico & 24276 \\
\hline
\end{tabular}


En la Figura NRo. 38 se observa una ventana de salida que muestra algunos parámetros descriptivos del circuito 2.

FIgURA NRO. 38. Parámetros descriptivos del circuito 2.

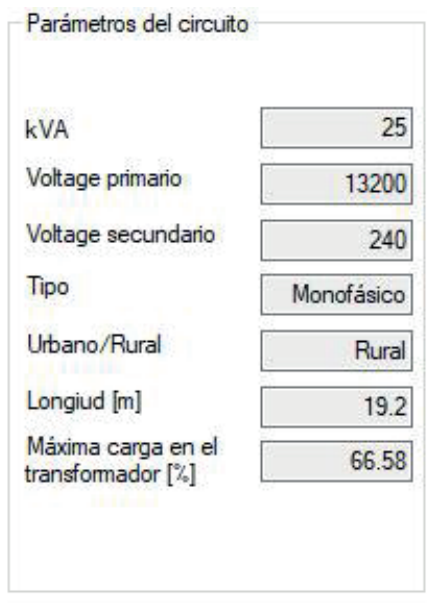

En la Figura NRO. 38 se puede observar que el circuito presenta una cargabilidad del $66.58 \%$ en la hora de demanda máxima y en la FiguRa NRO. 39 se observa la energía anual de entrada al transformador, el consumo por alumbrado público y las pérdidas técnicas del circuito 2 con su respectivo porcentaje de pérdidas desglosadas por componente.

FiguRA NRO. 39. Energía anual y pérdidas técnicas del circuito 2.

\begin{tabular}{|c|c|c|}
\hline \multicolumn{3}{|c|}{$\begin{array}{l}\text { Energia anual } \\
\text { De entrada al trans }\end{array}$} \\
\hline & & 114781.92 \\
\hline \multicolumn{3}{|c|}{ Alumbrado público [kWh] } \\
\hline \multicolumn{3}{|c|}{ Pérdidas Técnicas } \\
\hline & [kWh] & {$[\%]$} \\
\hline Transformador & 1686.77 & 1.47 \\
\hline Acometidas & 474.44 & 0.41 \\
\hline Medidores & 210.24 & 0.18 \\
\hline Red & 366.92 & 0.32 \\
\hline Total & 2738.37 & 2.39 \\
\hline
\end{tabular}


En este caso, se observa que el elemento con mayor porcentaje de pérdidas técnicas es el transformador de distribución con un porcentaje del $1.47 \%$. Esto se debe a la topología del circuito el cual consta de pocos tramos de red dado que los usuarios están conectados directamente a la salida del transformador. En segundo lugar, se encuentran las acometidas con un $0.41 \%$, y finalmente la red de distribución y los medidores con un porcentaje de pérdidas del $0.32 \%$ y $0.18 \%$, respectivamente.

En la Figura NRO. 40 se observa la máxima cargabilidad y la máxima caída de tensión para el circuito 2 .

Figura NRO. 40. Máxima cargabilidad y caída de tensión para el circuito 2.

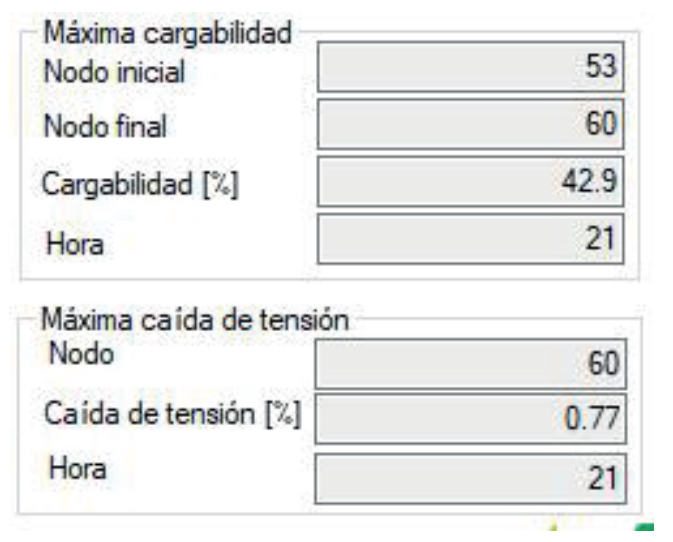

Como se puede observar, la hora de mayor cargabilidad para el circuito es la hora 21. En esta, se alcanza un valor del 42.9 $\%$ entre los nodos 53 y 60 . Por otra parte, la caída de tensión máxima es del $0.77 \%$ en el nodo 60 , el cual corresponde al nodo final del tramo de red más cargado.

\subsection{Circuito de prueba 3}

La Figura NRO. 41 muestra la topología del circuito simulado y la TABLA NRO. 19 presenta la información de los usuarios conectados al circuito de distribución 3. 
FIGURA NRO. 41. Topología del circuito 3.

Transformador $1 \mathrm{~F}-37.5 \mathrm{kVA}$

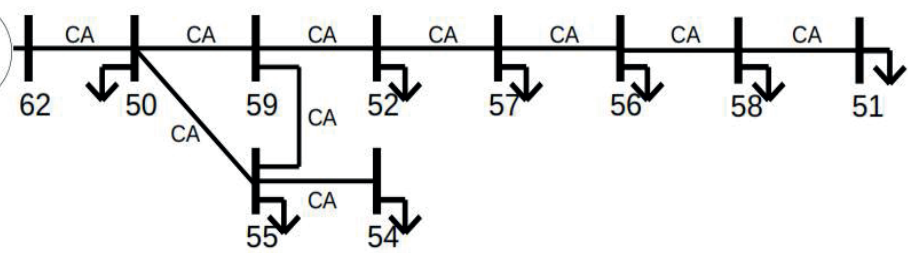

TABLA NRO. 19. Información de usuarios conectados al circuito 3.

\begin{tabular}{|c|c|c|c|c|c|}
\hline Nodo & $\begin{array}{c}\text { Calibre } \\
\text { Acometida }\end{array}$ & $\begin{array}{c}\text { Longitud } \\
\text { Acometida } \\
\text { [m] }\end{array}$ & Conexión & $\begin{array}{l}\text { Tipo de } \\
\text { Medidor }\end{array}$ & $\begin{array}{c}\text { Consumo } \\
\text { anual } \\
\text { kWh }\end{array}$ \\
\hline 50 & 8 & 17.1 & $\mathrm{BN}$ & Mecánico & 534 \\
\hline 50 & 8 & 7.5 & $\mathrm{CN}$ & Mecánico & 318 \\
\hline 51 & 8 & 13.6 & $\mathrm{BN}$ & Mecánico & 6600 \\
\hline 51 & 8 & 16.6 & $\mathrm{BN}$ & Mecánico & 4914 \\
\hline 52 & 8 & 6.2 & $\mathrm{BN}$ & Mecánico & 5112 \\
\hline 55 & 8 & 9.5 & $\mathrm{BN}$ & Mecánico & 15420 \\
\hline 55 & 8 & 6 & $\mathrm{BN}$ & Mecánico & 7566 \\
\hline 55 & 8 & 6.1 & $\mathrm{BN}$ & Mecánico & 8706 \\
\hline 56 & 8 & 4.1 & $\mathrm{BN}$ & Mecánico & 3486 \\
\hline 56 & 8 & 5.4 & $\mathrm{BN}$ & Mecánico & 5592 \\
\hline 58 & 8 & 8.2 & $\mathrm{BN}$ & Mecánico & 10026 \\
\hline 58 & 8 & 6.7 & $\mathrm{BN}$ & Mecánico & 8340 \\
\hline 58 & 8 & 10.2 & $\mathrm{BN}$ & Mecánico & 8802 \\
\hline 50 & 8 & 6 & $\mathrm{BN}$ & Mecánico & 6414 \\
\hline 50 & 8 & 4.6 & $\mathrm{BN}$ & Mecánico & 7164 \\
\hline 50 & 8 & 3.9 & $\mathrm{BN}$ & Mecánico & 8616 \\
\hline 50 & 8 & 3.5 & $\mathrm{BN}$ & Mecánico & 12894 \\
\hline 50 & 8 & 2.9 & $\mathrm{BN}$ & Mecánico & 3024 \\
\hline 55 & 8 & 12 & $\mathrm{CN}$ & Mecánico & 1560 \\
\hline 56 & 8 & 12 & $\mathrm{CN}$ & Electrónico & 5106 \\
\hline 56 & 8 & 14 & $\mathrm{CN}$ & Mecánico & 3444 \\
\hline 57 & 8 & 11 & $\mathrm{CN}$ & Mecánico & 3738 \\
\hline 56 & 8 & 18 & $\mathrm{BN}$ & Mecánico & 4608 \\
\hline 56 & 8 & 15 & $\mathrm{CN}$ & Mecánico & 3066 \\
\hline 56 & 8 & 18 & $\mathrm{CN}$ & Mecánico & 7296 \\
\hline 55 & 8 & 15 & $\mathrm{BN}$ & Mecánico & 9288 \\
\hline 54 & 8 & 12 & $\mathrm{BN}$ & Mecánico & 5718 \\
\hline 55 & 8 & 15 & $\mathrm{BN}$ & Mecánico & 7134 \\
\hline 54 & 8 & 10.2 & $\mathrm{BN}$ & Mecánico & 4500 \\
\hline 51 & 8 & 12 & $\mathrm{CN}$ & Mecánico & 156 \\
\hline
\end{tabular}


En la Figura NRO. 42 se observa una ventana de salida que muestra algunos parámetros descriptivos del circuito 3 .

Figura NRO. 42. Parámetros descriptivos del circuito 3.

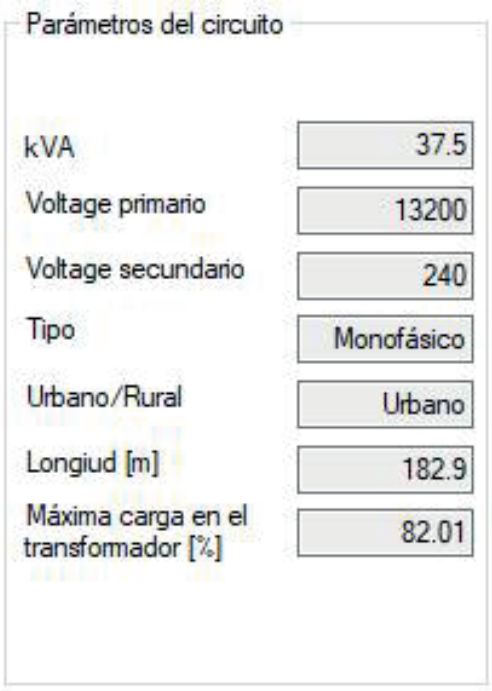

En la Figura NRO. 42 se puede observar que el transformador alcanza una cargabilidad del $82.01 \%$ en la hora de demanda máxima y en la FiguRA NRO. 43 se observa la energía anual de entrada al transformador, el consumo por alumbrado público y las pérdidas técnicas del circuito 3 con su respectivo porcentaje de pérdidas desglosadas por componente.

FiguRA NRO. 43. Energía anual y pérdidas técnicas del circuito 3.

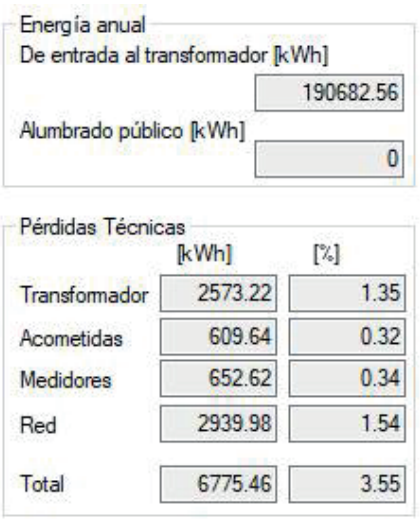


En el circuito 3 se observa que el elemento con mayor porcentaje de pérdidas técnicas es la red de distribución con un porcentaje del $1.54 \%$. En segundo lugar, se encuentran el transformador de distribución con un $1.35 \%$, y finalmente los medidores y acometidas con un porcentaje de pérdidas del 0.34 $\%$ y $0.32 \%$, respectivamente.

En la Figura NRO. 44 se observa la máxima cargabilidad y la máxima caída de tensión para el circuito 3.

FiguRA NRO. 44. Máxima cargabilidad y caída de tensión para el circuito 3.

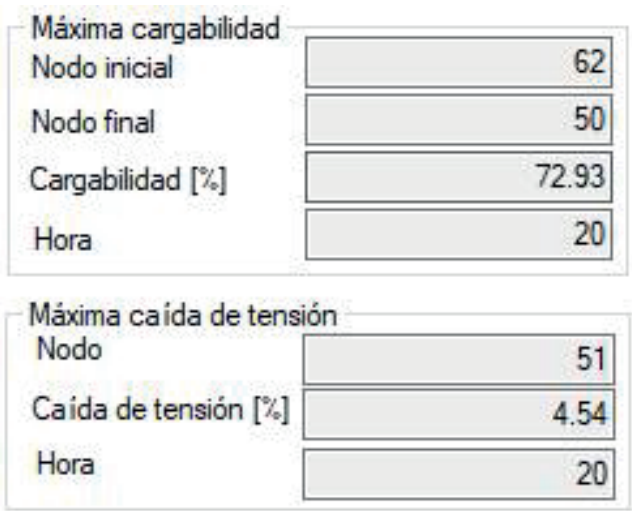

Como se puede observar, la hora de mayor cargabilidad para el circuito es la 20. A esta hora, la cargabilidad de la red es del $72.93 \%$ entre los nodos 62 y 50, los cuales corresponden al tramo de red a la salida del transformador. Por otra parte, la caída de tensión máxima es del $4.54 \%$ en el nodo 51, el cual es la cola del circuito. El circuito se encuentra con buena carga y las pérdidas técnicas se concentran en la red y el transformador.

\subsection{Circuito de prueba 4}

La Figura NRO. 45 muestra la topología del circuito simulado y la TABLA NRO. 20 presenta la información de los usuarios conectados al circuito de distribución 4 . 
Figura NRO. 45. Topología del circuito 4.

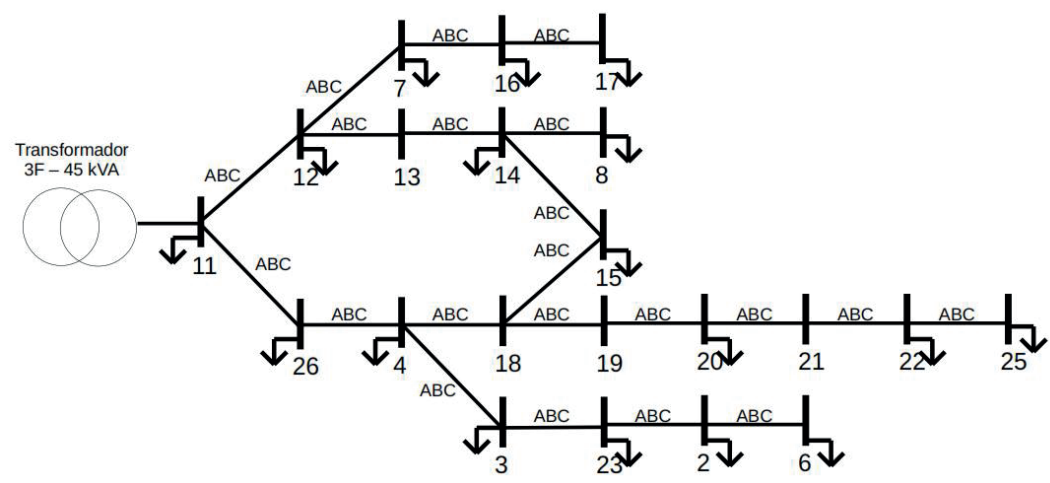

TABLA NRO. 20. Información de usuarios conectados al circuito 4.

\begin{tabular}{|c|c|c|c|c|c|}
\hline Nodo & $\begin{array}{c}\text { Calibre } \\
\text { Acometida }\end{array}$ & $\begin{array}{c}\text { Longitud } \\
\text { Acometida } \\
{[\mathbf{m}]}\end{array}$ & Conexión & $\begin{array}{c}\text { Tipo de } \\
\text { Medidor }\end{array}$ & $\begin{array}{c}\text { Consumo } \\
\text { anual } \\
\mathbf{k W h}\end{array}$ \\
\hline 2 & 8 & 12.7 & BN & Mecánico & 3807 \\
\hline 3 & 8 & 10.8 & BN & Mecánico & 3397.5 \\
\hline 3 & 8 & 18 & AN & Mecánico & 3816 \\
\hline 4 & 8 & 16.2 & CN & Mecánico & 7717.5 \\
\hline 4 & 8 & 12 & ABC & Mecánico & 8104.5 \\
\hline 6 & 8 & 6.2 & AN & Electrónico & 5058 \\
\hline 7 & 8 & 8.9 & AN & Mecánico & 1647 \\
\hline 7 & 8 & 16.5 & BN & Mecánico & 5206.5 \\
\hline 7 & 8 & 9.2 & CN & Electrónico & 3618 \\
\hline 7 & 8 & 3.1 & AN & Mecánico & 3730.5 \\
\hline 8 & 8 & 4.5 & AN & Mecánico & 4500 \\
\hline 11 & 8 & 5.3 & BN & Mecánico & 4801.5 \\
\hline 12 & 8 & 4.2 & BN & Mecánico & 6273 \\
\hline 12 & 8 & 6.3 & AN & Mecánico & 3699 \\
\hline 12 & 8 & 8.6 & CN & Mecánico & 5067 \\
\hline 14 & 8 & 4.9 & AN & Mecánico & 1935 \\
\hline 14 & 8 & 5.5 & CN & Mecánico & 2988 \\
\hline 15 & 8 & 3.5 & CN & Electrónico & 3186 \\
\hline 16 & 8 & 15.7 & AN & Mecánico & 9369 \\
\hline 16 & 8 & 11.4 & AN & Mecánico & 6889.5 \\
\hline 16 & 8 & 16.1 & CN & Mecánico & 3681 \\
\hline 17 & 8 & 13.4 & AN & Mecánico & 2587.5 \\
\hline 17 & 8 & 5.4 & AN & Mecánico & 7510.5 \\
\hline 17 & 8 & 14.1 & BN & Mecánico & 4077 \\
\hline 20 & 8 & 7.1 & BN & Mecánico & 4419 \\
\hline & & & & & \\
\hline
\end{tabular}


En la Figura NRo. 46 se observa una ventana de salida que muestra algunos parámetros descriptivos del circuito 4 .

FiguRA NRO. 46. Parámetros descriptivos del circuito 4.

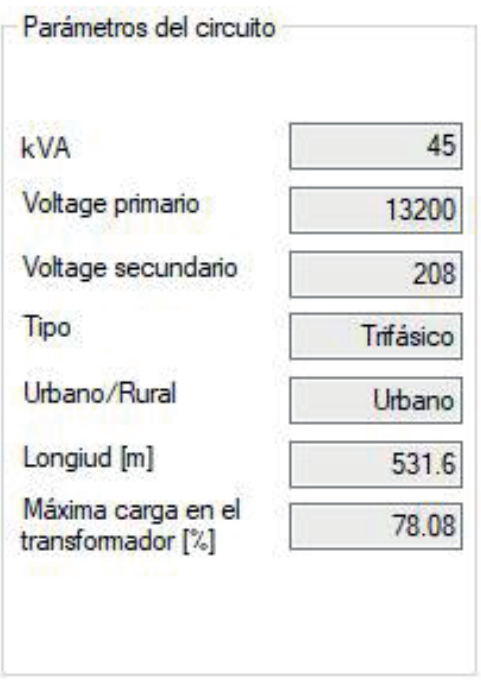

En la Figura NRO. 46 se observa que el transformador presenta una cargabilidad del $78.08 \%$ en la hora de demanda máxima y en la Figura NRO. 47 se observa la energía anual de entrada al transformador, el consumo por alumbrado público y las pérdidas técnicas del circuito 4 con su respectivo porcentaje de pérdidas desglosadas por componente.

FigURA NRO. 47. Energía anual y pérdidas técnicas del circuito 4.

\begin{tabular}{|c|c|c|}
\hline $\begin{array}{l}\text { Energia anual } \\
\text { De entrada al } t\end{array}$ & nsformador & Wh] \\
\hline & & 194383.16 \\
\hline Alumbrado púb & o [kWh] & \\
\hline & & 5775.6 \\
\hline Pérdidas Técni & & \\
\hline & [kWh] & {$[\%]$} \\
\hline Transformador & 3222.72 & 1.66 \\
\hline Acometidas & 1415.34 & 0.73 \\
\hline Medidores & 643.86 & 0.33 \\
\hline Red & 2539.2 & 1.31 \\
\hline Total & 7821.12 & 4.02 \\
\hline
\end{tabular}


En el circuito 4, se observa que el elemento con mayor porcentaje de pérdidas técnicas es el transformador de distribución con un porcentaje del $1.66 \%$. En segundo lugar, se encuentra la red de distribución con un $1.31 \%$, y finalmente las acometidas y los medidores con un porcentaje de pérdidas del $0.73 \%$ y $0.33 \%$, respectivamente.

En la Figura NRO. 48 se observa la máxima cargabilidad y la máxima caída de tensión para el circuito 4 .

FIgURA NRO. 48. Máxima cargabilidad y caída de tensión para el circuito 4.

\begin{tabular}{|c|c|}
\hline \multicolumn{2}{|l|}{ Máxima cargabilidad } \\
\hline Nodo inicial & 11 \\
\hline Nodo final & 26 \\
\hline Cargabilidad [\%] & 36.27 \\
\hline Hora & 20 \\
\hline \multicolumn{2}{|c|}{ Máxima caida de tensión } \\
\hline Nodo & 25 \\
\hline Caida de tensión [\%] & 5.96 \\
\hline Hora & 20 \\
\hline
\end{tabular}

Como se puede observar, la hora de mayor cargabilidad para el circuito es la 20. A esta hora la cargabilidad de la red es del $36.27 \%$ entre los nodos 11 y 26 . Por otra parte, la caída de tensión máxima es del $5.96 \%$ en el nodo 25 .

\subsection{Resultados a nivel del sistema de distribución secundaria}

Se consideró un sistema de distribución con solamente 4 transformadores. El año simulado tendrá 295 días ordinarios y 70 días festivos. Los resultados entregados por los flujos de carga se muestran en la TABLA NRO. 21. 
TABLA NRO. 21. Resultados de los circuitos para los días festivos y ordinarios.

\begin{tabular}{|c|c|c|c|c|c|c|c|c|}
\hline \multirow[b]{2}{*}{ Circuito } & \multicolumn{4}{|c|}{ Días festivos } & \multicolumn{4}{|c|}{ Días ordinarios } \\
\hline & 1 & 2 & 3 & 4 & 1 & 2 & 3 & 4 \\
\hline kVA & 75 & 25 & 37.5 & 45 & 75 & 25 & 37.5 & 45 \\
\hline Tipo & Trifásico & Monofásico & Monofásico & Trifásico & Trifásico & Monofásico & Monofásico & Trifásico \\
\hline Topología & Radial & Radial & Mallado & Mallado & Radial & Radial & Mallado & Mallado \\
\hline Ubicación & Urbano & Rural & Urbano & Urbano & Urbano & Rural & Urbano & Urbano \\
\hline Zona operativa & 2 & 2 & 3 & 1 & 2 & 2 & 3 & 1 \\
\hline Longitud de red & 414 & 19.2 & 182.9 & 531.6 & 414 & 19.2 & 182.9 & 531.6 \\
\hline $\begin{array}{l}\text { Energía de } \\
\text { entrada }[\mathrm{kWh}]\end{array}$ & 422.23 & 306.86 & 501.17 & 532.04 & 562.78 & 316.27 & 527.45 & 532.67 \\
\hline Per. Red [kWh] & 7.32 & 0.96 & 7.53 & 6.97 & 12.81 & 1.01 & 8.17 & 6.95 \\
\hline Per. Med. [kWh] & 1.284 & 0.576 & 1.788 & 1.764 & 1.284 & 0.576 & 1.788 & 1.764 \\
\hline Per. Aco. [kWh] & 0.74 & 1.25 & 1.57 & 3.89 & 1.32 & 1.31 & 1.7 & 3.87 \\
\hline Per. Trans. [kWh] & 7.92 & 4.53 & 6.75 & 8.82 & 9.13 & 4.64 & 7.12 & 8.83 \\
\hline $\begin{array}{l}\text { \% Pérdidas } \\
\text { circuito }\end{array}$ & 4.01 & 2.35 & 3.47 & 3.97 & 4.29 & 2.35 & 3.51 & 3.96 \\
\hline \% Per. Red & 1.7 & 0.31 & 1.48 & 1.29 & 2.24 & 0.32 & 1.53 & 1.28 \\
\hline $\begin{array}{l}\% \text { Per. } \\
\text { Acometidas }\end{array}$ & 0.17 & 0.4 & 0.31 & 0.72 & 0.23 & 0.41 & 0.32 & 0.72 \\
\hline$\%$ Per. Medidores & 0.3 & 0.18 & 0.35 & 0.33 & 0.22 & 0.18 & 0.33 & 0.33 \\
\hline$\%$ Per. Transf. & 1.84 & 1.45 & 1.33 & 1.63 & 1.6 & 1.45 & 1.33 & 1.63 \\
\hline $\begin{array}{l}\text { Máxima } \\
\text { Cargabilidad del } \\
\text { Transformador }\end{array}$ & 31.45 & 65.42 & 78.39 & 76.18 & 39.52 & 66.58 & 82.01 & 78.08 \\
\hline Peor Regulación & 4.18 & 0.76 & 4.35 & 5.86 & 5.22 & 0.77 & 4.54 & 5.96 \\
\hline $\begin{array}{l}\text { Nodo con peor } \\
\text { regulación }\end{array}$ & 31 & 60 & 51 & 25 & 31 & 60 & 51 & 25 \\
\hline $\begin{array}{l}\text { Hora de peor } \\
\text { regulación }\end{array}$ & 21 & 21 & 20 & 20 & 21 & 21 & 20 & 20 \\
\hline $\begin{array}{l}\text { Máxima } \\
\text { cargabilidad de } \\
\text { línea }\end{array}$ & 47 & 42.16 & 69.72 & 35.4 & 58.92 & 42.9 & 72.93 & 36.27 \\
\hline Nodo inicial & 5 & 5 & 5 & 5 & 5 & 5 & 5 & 5 \\
\hline Nodo final & 33 & 60 & 50 & 26 & 33 & 60 & 50 & 26 \\
\hline $\begin{array}{l}\text { Hora con mayor } \\
\text { cargabilidad de } \\
\text { línea }\end{array}$ & 21 & 21 & 20 & 20 & 21 & 21 & 20 & 20 \\
\hline
\end{tabular}

La TABla NRO. 21 presenta los resultados globales de las pérdidas técnicas del sistema (considerando los 4 transformadores de distribución). Adicionalmente, se presenta las pérdidas técnicas por componente del sistema tanto en $\mathrm{kWh}$ como en porcentaje.

TABLA NRO. 22. Pérdidas técnicas globales del sistema de distribución.

\begin{tabular}{|c|c|c|c|c|c|}
\hline $\begin{array}{c}\text { Pérdidas } \\
\text { Técnicas } \\
{[\mathbf{M W h}]}\end{array}$ & $\begin{array}{c}\text { Pérdidas } \\
\text { Técnicas } \\
{[\%]}\end{array}$ & $\begin{array}{c}\text { Pérdidas en el } \\
\text { Transformador } \\
{[\mathbf{M W h}]}\end{array}$ & $\begin{array}{c}\text { Pérdidas en el } \\
\text { Transformador } \\
{[\%]}\end{array}$ & $\begin{array}{c}\text { Pérdidas } \\
\text { en la Red } \\
{[\mathbf{M W h}]}\end{array}$ & $\begin{array}{c}\text { Pérdidas } \\
\text { en la Red } \\
{[\%]}\end{array}$ \\
\hline 25.78 & 3.71 & 10.70 & 1.54 & 10.15 & 1.46 \\
\hline
\end{tabular}

\begin{tabular}{|c|c|c|c|c|}
\hline $\begin{array}{c}\text { Pérdidas } \\
\text { en la Acometida } \\
{[\mathbf{M W h}]}\end{array}$ & $\begin{array}{c}\text { Pérdidas en } \\
\text { la Acometida } \\
{[\%]}\end{array}$ & $\begin{array}{c}\text { Pérdidas en el } \\
\text { Medidor } \\
{[\mathbf{M W h}]}\end{array}$ & $\begin{array}{c}\text { Pérdidas en el } \\
\text { Medidor } \\
{[\%]}\end{array}$ & $\begin{array}{c}\text { Energía } \\
\text { de entrada } \\
{[\mathbf{M W h}]}\end{array}$ \\
\hline 2.92 & 0.42 & 1.94 & 0.28 & 695.43 \\
\hline
\end{tabular}


También, se presentan las pérdidas técnicas desglosadas por ubicación (urbano o rural en la TABLA NRO. 23), tipo de transformador (monofásico o trifásico en la TABLA NRO. 24), topología de red (radial o mallada en la TABLA NRo. 25) y zona geográfica (en la TABLA NRO. 26). Para calcular los porcentajes, se tomó como referencia toda la energía de entrada del sistema de distribución.

TABLA NRO. 23. Resultados de pérdidas técnicas por localización urbano/rural.

\begin{tabular}{|c|c|c|c|c|c|}
\hline Localización & $\begin{array}{c}\text { Pérdidas } \\
\text { técnicas } \\
{[\mathbf{M W h}]}\end{array}$ & $\begin{array}{c}\text { Pérdidas } \\
\text { técnicas [\%] }\end{array}$ & $\begin{array}{c}\text { Pérdidas en el } \\
\text { Transformador } \\
{[\mathbf{M W h}]}\end{array}$ & $\begin{array}{c}\text { Pérdidas en el } \\
\text { Transformador } \\
{[\%]}\end{array}$ & $\begin{array}{c}\text { Pérdidas en } \\
\text { la Red } \\
{[\mathbf{M W h}]}\end{array}$ \\
\hline Urbano & 23.05 & 3.97 & 9.04 & 1.56 & 9.77 \\
\hline Rural & 2.74 & 2.39 & 1.69 & 1.47 & 0.37 \\
\hline
\end{tabular}

\begin{tabular}{|c|c|c|c|c|c|}
\hline $\begin{array}{c}\text { Pérdidas en la } \\
\text { Red [\%] }\end{array}$ & $\begin{array}{c}\text { Pérdidas en la } \\
\text { Acometida } \\
{[\mathbf{M W h}]}\end{array}$ & $\begin{array}{c}\text { Pérdidas en la } \\
\text { Acometida } \\
{[\%]}\end{array}$ & $\begin{array}{c}\text { Pérdidas en el } \\
\text { Medidor } \\
{[\mathbf{M W h ]}}\end{array}$ & $\begin{array}{c}\text { Pérdidas en el } \\
\text { Medidor [\%] }\end{array}$ & $\begin{array}{c}\text { Energía de } \\
\text { entrada [MWh] }\end{array}$ \\
\hline 1.68 & 2.46 & 0.30 & 1.76 & 0.42 & 580.64 \\
\hline 0.32 & 0.47 & 0.18 & 0.21 & 0.41 & 114.78 \\
\hline
\end{tabular}

En la Figura NRO. 49 se observan las pérdidas totales por ubicación urbana y rural.

Figura NRO. 49. Pérdidas totales por locación.

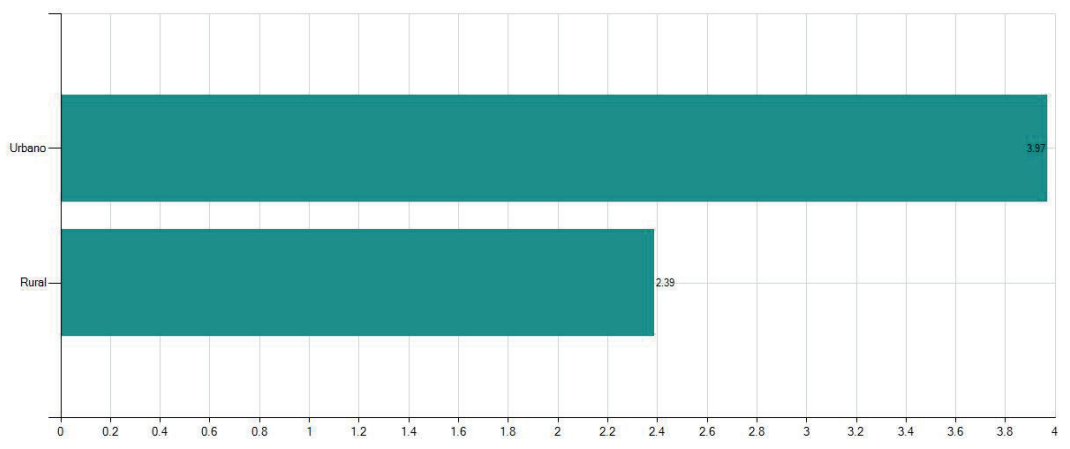


TABla NRO. 24. Pérdidas técnicas para circuitos por tipo de transformador monofásico/trifásico

\begin{tabular}{|c|c|c|c|c|c|}
\hline $\begin{array}{c}\text { Tipo de } \\
\text { Transformador }\end{array}$ & $\begin{array}{l}\text { Pérdidas } \\
\text { técnicas } \\
\text { [MWh] }\end{array}$ & $\begin{array}{c}\text { Pérdidas } \\
\text { técnicas }[\%]\end{array}$ & $\begin{array}{c}\text { Pérdidas en el } \\
\text { Transformador } \\
{[M W h]}\end{array}$ & $\begin{array}{c}\text { Pérdidas en el } \\
\text { Transformador } \\
{[\%]}\end{array}$ & $\begin{array}{c}\text { Pérdidas en } \\
\text { la Red } \\
\text { [MWh] }\end{array}$ \\
\hline Monofásico & 9.51 & 3.11 & 4.26 & 1.39 & 3.30 \\
\hline Trifásico & 16.27 & 4.17 & 6.47 & 1.66 & 6.83 \\
\hline $\begin{array}{c}\text { Pérdidas en la } \\
\text { Red }[\%]\end{array}$ & $\begin{array}{c}\text { Pérdidas en la } \\
\text { Acometida } \\
\text { [MWh] }\end{array}$ & $\begin{array}{c}\text { Pérdidas en la } \\
\text { Acometida [\%] }\end{array}$ & $\begin{array}{c}\text { Pérdidas en el } \\
\text { Medidor } \\
\text { [MWh] }\end{array}$ & $\begin{array}{l}\text { Pérdidas en el } \\
\text { Medidor [\%] }\end{array}$ & $\begin{array}{c}\text { Energía de } \\
\text { entrada [MWh] }\end{array}$ \\
\hline 1.08 & 1.08 & 0.28 & 0.86 & 0.35 & 305.46 \\
\hline 1.75 & 1.85 & 0.29 & 1.11 & 0.48 & 389.96 \\
\hline
\end{tabular}

En la Figura NRO. 50 se observa las pérdidas totales por tipo de transformador.

FiguRA NRO. 50. Pérdidas totales por tipo de transformador.

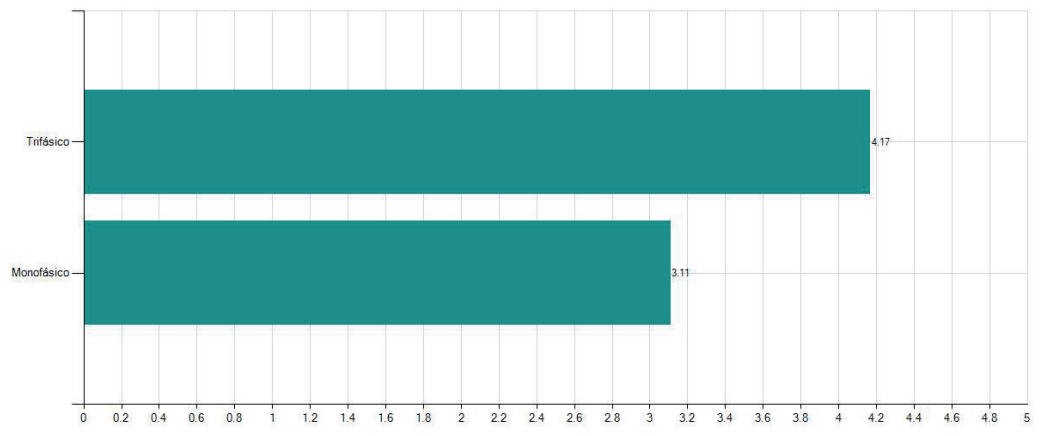

TABLA NRO. 25. Resultados de pérdidas técnicas por topología radial/ mallado.

\begin{tabular}{|c|c|c|c|c|c|}
\hline Topología & $\begin{array}{c}\text { Pérdidas } \\
\text { técnicas } \\
{[\mathbf{M W h}]}\end{array}$ & $\begin{array}{c}\text { Pérdidas } \\
\text { técnicas [\%] }\end{array}$ & $\begin{array}{c}\text { Pérdidas en el } \\
\text { Transformador } \\
{[\mathbf{M W h}]}\end{array}$ & $\begin{array}{c}\text { Pérdidas en el } \\
\text { Transformador } \\
{[\mathbf{\%}]}\end{array}$ & $\begin{array}{c}\text { Pérdidas en } \\
\text { la Red } \\
{[\mathbf{M W h}]}\end{array}$ \\
\hline Radial & 11.19 & 3.60 & 4.93 & 1.62 & 4.66 \\
\hline Mallado & 14.60 & 3.79 & 5.80 & 1.49 & 5.48 \\
\hline
\end{tabular}

\begin{tabular}{|c|c|c|c|c|c|}
\hline $\begin{array}{c}\text { Pérdidas en la } \\
\text { Red [\%] }\end{array}$ & $\begin{array}{c}\text { Pérdidas en la } \\
\text { Acometida } \\
{[\mathbf{M W h}]}\end{array}$ & $\begin{array}{c}\text { Pérdidas en la } \\
\text { Acometida } \\
{[\%]}\end{array}$ & $\begin{array}{c}\text { Pérdidas en el } \\
\text { Medidor } \\
{[\mathbf{M W h}]}\end{array}$ & $\begin{array}{c}\text { Pérdidas en el } \\
\text { Medidor [\%] }\end{array}$ & $\begin{array}{c}\text { Energía de } \\
\text { entrada [MWh] }\end{array}$ \\
\hline 1.53 & 0.91 & 0.22 & 0.68 & 0.30 & 310.36 \\
\hline 1.41 & 2.02 & 0.33 & 1.23 & 0.52 & 385.06 \\
\hline
\end{tabular}

En la Figura NRO. 51 se observa las pérdidas totales por tipo de topología de red (radial y mallado). 
Figura NRO. 51. Pérdidas totales por tipo de topología de red.

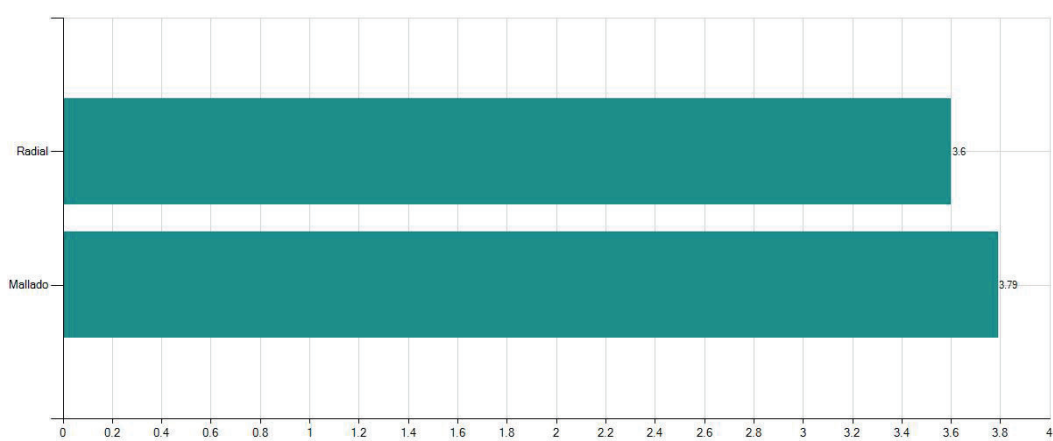

TABLA NRO. 26. Resultados de pérdidas técnicas por zona geográfica.

\begin{tabular}{|c|c|c|c|c|c|}
\hline Zona & $\begin{array}{c}\text { Pérdidas } \\
\text { técnicas } \\
{[\mathbf{M W h}]}\end{array}$ & $\begin{array}{c}\text { Pérdidas } \\
\text { técnicas [\%] }\end{array}$ & $\begin{array}{c}\text { Pérdidas en el } \\
\text { Transformador } \\
{[\mathbf{M W h}]}\end{array}$ & $\begin{array}{c}\text { Pérdidas en el } \\
\text { Transformador } \\
{[\mathbf{\%}]}\end{array}$ & $\begin{array}{c}\text { Pérdidas en } \\
\text { [a Red } \\
{[\mathbf{M W h}]}\end{array}$ \\
\hline 1 & 7.82 & 4.02 & 3.22 & 1.66 & 2.54 \\
\hline 2 & 11.19 & 3.60 & 4.93 & 1.59 & 4.66 \\
\hline 3 & 6.77 & 3.55 & 2.57 & 1.35 & 2.94 \\
\hline
\end{tabular}

\begin{tabular}{|c|c|c|c|c|c|}
\hline $\begin{array}{c}\text { Pérdidas en la } \\
\text { Red [\%] }\end{array}$ & $\begin{array}{c}\text { Pérdidas en la } \\
\text { Acometida } \\
{[\mathbf{M W h}]}\end{array}$ & $\begin{array}{c}\text { Pérdidas en la } \\
\text { Acometida } \\
{[\mathbf{\%}]}\end{array}$ & $\begin{array}{c}\text { Pérdidas en el } \\
\text { Medidor } \\
{[\mathbf{M W h}]}\end{array}$ & $\begin{array}{c}\text { Pérdidas en el } \\
\text { Medidor [\%] }\end{array}$ & $\begin{array}{c}\text { Energía de } \\
\text { entrada [MWh] }\end{array}$ \\
\hline 1.31 & 1.41 & 0.33 & 0.64 & 0.73 & 194.38 \\
\hline 1.50 & 0.91 & 0.22 & 0.68 & 0.29 & 310.36 \\
\hline 1.54 & 0.61 & 0.34 & 0.65 & 0.32 & 190.68 \\
\hline
\end{tabular}

En la FiguRA NRO. 52 se observa las pérdidas totales por tipo de zona geográfica.

Figura NRO. 52. Pérdidas totales por tipo de zona geográfica.

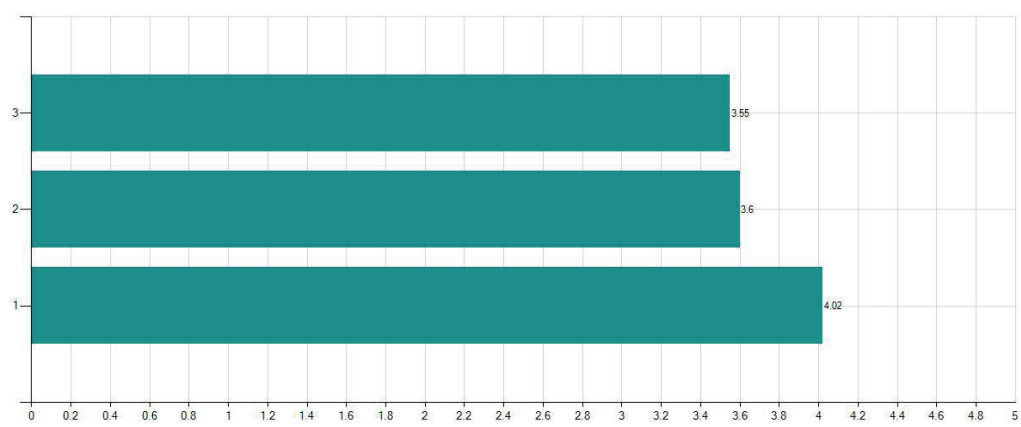




\section{Referencias bibliográficas}

1. Comisión de Regulación de Energía y Gas. (2018, Ene. 28) ResoluciónCREG015de2018[Online].Avaible:http://apolo.creg. gov.co/Publicac.nsf/1c09d18d2d5ffb5b05256eee00709c02/ 65f1aaf1d57726a9052582 2900064dac/\$FILE/ Creg015-2018. pdf.

2. R. Jiménez, T. Serebrisky y J. Mercado, Electricidad perdida: Dimensionando las pérdidas de electricidad en los sistemas de transmisión en América Latina y el Caribe, Washington: BID, 2014.

3. W . H. Kersting, Distribution System Modeling and Analysis, Boca Raton, FL: CRC Press LLC, 2000.

4. S . S. Miller et al., "Chapter 4 System Modeling,» in IEEE Recommended Practice for Industrial and Commercial Power Systems Analysis (Brown Book), L. G. Jackson, ch., IEEE: New York, NY, 1998, pp. 43-102.

5. W . W. Price et al., «Load Representation for Dynamics Perfomance Analysis,» IEEE Trans. Power Syst., vol. 8, n. 2, May., 1993, pp. 472-482.

6. T ransformadores monofásicos autorefrigerados y sumergidos en líquido. Corriente sin carga, pérdidas y tensión de corto circuito, NTC 818, 1995.

7. A . H. Escobar Zuluaga, R. A. Gallego Rendón, M. Granada Echeverri y, Reducción de pérdidas por etapas en sitemas de distribución, Pereira (Colombia): Editorial Universidad Tecnológica de Pereira, 2006. 
8. Transformadores trifásicos autorefrigerados y sumergidos en líquido. Corriente sin carga, pérdidas y tensión de cortocircuito, NTC 819, 1995.

9. Universidad Tecnológica de Pereira, «Tomo I - Cálculo de pérdidas técnicas en el Nivel de tensión 1,», UTP-CREG, Convenio Interadministrativo, Doc. 5112326202 - 03, Jun. 30, 2010.

10.Equipos de medición de energía eléctrica (C.A.). Requisitos particulares. Medidores estáticos de energía activa (clases 0,2 s y 0,5s), NTC 2147, 2003.

11.Equipos de medición de energía eléctrica (C.A.), requisitos particulares, medidores electromecánicos de energía activa (clases 0.5, 1 y 2), NTC 2288, 2003.

12. Equipos de medición de energía eléctrica (C.A.). Requisitos particulares. Medidores estáticos de energía activa (clases 1 y 2), NTC 4052, 2003.

13.E quipo para medidores de energia electrica (c.a) requisitos particulares. Requisitos de tension y consumo de potencia, NTC 4649, 1999. 
Uno de los objetivos de las empresas de distribución es reducir sus niveles de pérdidas técnicas para alcanzar la eficiencia en el uso de los recursos y racionalizar las necesidades de inversión en infraestructura eléctrica. El índice de dichas pérdidas es un indicador de la gestión técnica de una empresa distribuidora; por lo que conocer su valor es crucial para la definición de políticas de operación del sistema. Para calcular el índice de pérdidas técnicas, se deben utilizar modelos adecuados de cada uno de los elementos que hacen parte del sistema, incluyendo el transformador, la red de distribución, los medidores, las acometidas y las cargas. Adicionalmente, se debe utilizar una metodología de flujo de carga que permita determinar las condiciones de operación en régimen permanente y considere las características particulares que tienen las redes de distribución secundarias.

El libro Pérdidas Técnicas en Redes Secundarias de Distribución de Energía Eléctrica presenta los resultados del proyecto de desarrollo tecnológico, denominado «Herramienta computacional para el cálculo de pérdidas técnicas en sistemas secundarios de distribución», registrado en la Vicerrectoría de Investigación, Innovación y Extensión de la Universidad Tecnológica de Pereira con el código 9-19-5. Este trabajo fue desarrollado por los grupos de investigación Laboratorio de Investigación en Desarrollo Eléctrico y Electrónico — LIDER-y Desarrollo en Investigación de Operaciones —DINOP—.

En su interior, se encuentran los modelos matemáticos de los componentes del sistema de distribución secundario, las metodologías matemáticas desarrolladas que permiten calcular el índice de pérdidas técnicas en redes radiales y en malla, y la descripción de los aplicativos desarrollados para tal fin. 